ELECTROMAGNETIC DECAY OF THE SECOND EXCITED STATE OF $\mathrm{C}^{12}$

Thesis by

Philip A. Seeger

In Partial Eulfument of the Requirements For the Degree of Doctor of Philosophy

California Institute of Tochnology

Pasadena, California 


\section{ACKNOW LEDGEMENTS}

I wish to thank Dr. R. W. Kavanagh for his great help in the conception, design, and execution of this experiment. I am also indebted to the entire staff of the Kellogg Radiation Laboratory, not only for many useful discussions and suggestions, but also for creating an atmosphere in which the work could be successfully carried out. 


\section{ABSTRACT}

It was desired to determine what fraction of $\mathrm{C}^{12}$ nuclel in the second excited state decay electromagnetically to the ground state instead of by alpha emission. The state was formed with the reaction $\mathrm{NI}^{14}(\mathrm{~d}, \mathrm{a}) \mathrm{C}^{12 *}$ by bombarding 2 thin vanadium nitride target on carbon foil with $1.8-\mathrm{MeV}$ deuterons. Recoil $\mathrm{C}^{12}$ nuclei were observed in coincidence with the alpha particles from the reaction. The alphas were detected with a surface-barrier counter set at the appropriate unique angle, and the recolling nuclei at $90^{\circ}$ were analyzed in momenturn with a magnetic spectrometer, in energy by a surfaco-barrier counter at the spectrometer focus, and in velocity by the time delay between the alpha and the $C^{12}$ counts. Pulses of 40 -nsec width were recorded photographically from a Tektronix type 555 dual-beam os cilloscope, providing a time resolution of $5 \mathrm{nsec}$, and also allowing pulse height analysis on the fast pulses. Of the $\mathrm{C}^{12}$ nuclei formed in the second excited state, $99.9+$ per cent decay by alpha emission to $\mathrm{Be}^{8}$ and are not detected; only those which decay to the $\mathrm{C}^{12}$ ground state by gamma or pair emission will be seen. Thus the experiment provides a direct measurement of $T_{\mathrm{rad}} / I_{\text {. The value of }} \mathrm{I}_{\mathrm{rad}}=$ $\left(I_{y}+\mathrm{I}_{e^{*}}\right)$ has astrophysical significance, determining the rate of $\mathrm{C}^{12}$ formation in the He-burning process in red giant stars. Analysis of 170 observed events gives $\mathrm{I}_{\mathrm{rad}} / \mathrm{Y}^{\prime}=(2.8 \pm 0.3) \times 10^{-4}$. Averaged with a value of $(3.3 \neq 0.9) \times 10^{-4}$ obtained by Alburger, and combined with a value of $I=(8 \pm 5) \mathrm{eV}$, the result is $I_{\mathrm{rad}}=(0.0024 \pm 0.0015) \mathrm{eV}$, which is two-and-one-half times the value used in previous astrophysical calculations. 


\section{TABLE OF CONTENTS}

I. INTRODUCTION
A. Astrophysical Significance. .......... 1
B. Previous Knowlodge .............. 3

II. EXPERIMENT; APPARATUS
A. Reasons for $U$ aing $\mathrm{N}^{14}(\mathrm{~d}, \alpha) \mathrm{C}^{12 *}$; Kinematics ... 5
B. Target Manufacture ............ 6
C. Recoll-Ion Detection ............ 7
D. Alpha Detection .............. 9
E. Coincidence Detection ............. 12

III. PROCEDURE AND RESULTS

A. Non-Coincident Recoil-Ion Spectra ....... 16

B. Non-Coincident Alpha Spectra . . . . . . . 16

C. Procedure for Recording Coincidences ...... 17

D. Energy and Time Normalieation........ 19

E. Determination of Number of Counts . . . . . . 22

E. Experimental Results ............ 24

IV. ANALYSIS OF RESULTS

A. Equation for $r_{\text {rad }} / \Gamma \ldots \ldots 26$

B. Population Factor, P ............. 28

C. Charge State Factor, C ........... 28

D. Geometry Factor, G............. 31

E. Thickness Factor, T. . . . . . . . . 34

F. Calculation of $\mathrm{r}_{\mathrm{rad}} / \mathrm{F} \ldots \ldots \ldots$

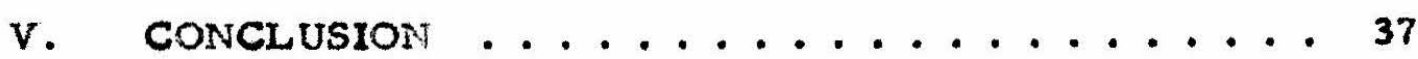


TABLE OF CONTENTS (Cont'd)

PAGE

APPENDICES

A. Thin Nitrogen Targets

1. Carbon-foil backing. .............. 40

2. Vanadium ovaporation .............. 4 40

3. Nitriding .................... 41

4. Composition. . . . . . . . . . . . . 42

B. Camera and Film Developing . . . . . . . . . . 44

C. Momentum of Gamma Rays

1. First excited state ................ 46

2. Second excited state ............. 47

D. Projection of a Rectangle in Spherical Co-ordinates . . 50 REFERENCES . . . . . . . . . . . . . 55

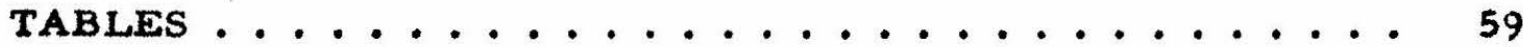

FIGURES ............................. 71 


\section{INTRODUCTION}

\section{A. Attrophysical Significance}

A star which has converted most of its hydrogen into helium will undergo gravitational contraction either until an instability arises or until the central temperature is raised to such a level that a new nuclear fuel is available as a source of energy. Salpeter $(1952,1957)$ has pointed out that at temperatures of the order of $10^{8}{ }^{\circ} \mathrm{K}$, the reactione

$$
\begin{aligned}
& H e^{4}+H e^{4}=B e^{8} \\
& B e^{3}+H e^{4}=C^{12 *}
\end{aligned}
$$

will produce small amounts of $\mathrm{Be}^{8}$ and $\mathrm{C}^{12^{*}}$ in dynamic equilibrium. The level of $\mathrm{C}^{12}$ involved has an interesting hitory, summarized by Cook (1957). When experiments established that the ground state of $B e^{8}$ is unbound by only $95 \pm 5 \mathrm{keV}$ (Fiemmendinger 1948. Tollestrup 1949). Salpeter (1952) calculated an equilibrium ratio of $\mathrm{Be}^{8}$ to $\mathrm{He}^{4}$ of the order of $10^{-9}$ for red giant atars. The effect of the resonance in $F e^{4}+3 e^{4}$ was to increase the helium-burning reaction rate compared to non-resonant calculations by Öplk $(1951,1953)$, but the Salpeter calculations atill did not give a fast enough reaction rate at $3 \times 10^{8}$ degrees. Hoyle (1954) predicted the existence of a level in $\mathrm{C}^{12}$ at $7.70 \mathrm{NieV}$, on the basis of considerations of reaction rates and of the relative abundances of $\mathrm{Fie}^{4}, \mathrm{C}^{12}$, and $\mathrm{O}^{16}$. At the time of Foyle' $\mathrm{g}$ pediction there was conflicting evidence regardiug the existence of such a state. A subsequent experiment (Foyle 1953, Dunbar 1953) using the reaction 
$N^{14}(d, \alpha) C^{12 *}$ with a high-resolution magnetic spectrometer to observe the alpha groups, clearly identified an alpha-particle group leading to the state in question. In Figure 1 (Lauritsen 1962) the state is shown at $7.656 \mathrm{MeV}$, very close to the position predicted by Hoyle. Cook (1957) showed that this otate does indeed decay into three alpha particles through an intermediate stage involving $\mathrm{He}^{4}$ and the ground atate of $\mathrm{Be}^{3}$, proving (by the general reversibility of nuclear reactions) that the reactions of equation 1 can occur.

If a $\mathrm{C}^{12 *}$ decays electromagnetically to the ground state, either by garnma emisaion, by paix ernission, or by gamma cascade through the fir at atate, the net result will be (Everling 1960)

$$
3 \mathrm{He}^{4} \rightarrow \mathrm{C}^{12}+7.275 \mathrm{MeV}
$$

The purpose of this experiment was to find the total electromagnetic decay width of the $7.656-\mathrm{MeV}$ state of $\mathrm{c}^{12}$.

If the probability of leakage to the ground state is amall compared to the alpha emission by $\mathrm{C}^{12 *}$ indicated in equation 1 , the equilibrium can be expressed (Salpeter 1957) by the law of mass action as for a triatomic molecule:

$$
n_{*}={ }_{\alpha}{ }^{3} \frac{\left(2 J_{*}+1\right)}{\left(2 J_{\alpha}+1\right)^{3}}\left(\frac{2 \pi \hbar^{2}}{M_{\alpha}^{k T}}\right)^{3} 3^{3 / 2} \exp \left(-\frac{E_{T}}{k T}\right) \text {. }
$$

where $n_{*}=$ number density of $c^{12 *}$.

$J_{*}=\operatorname{spin}$ of $\mathrm{C}^{12 *}=0$,

$n_{Q}=$ number density of $\mathrm{He}^{4}$.

$J_{Q}^{\alpha}=\operatorname{spin}$ of $\mathrm{He}^{4}=0$.

$\mathrm{M}_{\alpha}=$ mass of $\mathrm{He}^{4}$,

$\mathrm{E}_{r}=$ energy difference $\left(\mathrm{C}^{12 *}-3 \mathrm{He}\right) \mathrm{c}^{2}=372 \mathrm{keV}$ (Cook 1957). 
The rate of reaction 2 is then given by

$$
\mathbf{p}=\mathrm{n}_{*} \frac{\Gamma_{\text {rad }}}{\hbar}
$$

ance the mean lifetime for electromagnetic decay is $n / r_{r a d}$, where irad is the total radiative decay width. The energy generation per unit time and per unit mass is

$$
\epsilon=\frac{P Q}{P}=1.386 \times 10^{24} p_{5}^{2} x_{\alpha}^{3} f \frac{I_{\mathrm{rad}}}{T_{8}{ }^{3}} \exp \left(-\frac{43.2}{T_{8}}\right) \frac{\mathrm{erg}}{\mathrm{gec}}
$$

where $\rho_{5}=\rho \times 10^{-5}=$ density in $10^{5} \mathrm{~g} / \mathrm{cm}^{3}$.

$$
\begin{aligned}
& \mathrm{I}_{\mathrm{rad}} \text { is in eV, } \\
& x_{\alpha}=\text { mass fraction of He }{ }^{4}, \\
& T_{3}=\text { temperature in } 10^{8} \circ_{K_{n},} \\
& f \approx \exp \left[0.88\left(\frac{\rho_{5}}{T_{8}}\right)^{\frac{1}{2}}\right] .
\end{aligned}
$$

The factor $f$ is an electron screening correction (Salpeter 1954, $\mathrm{B}^{2} \mathrm{FH}$ 1957) necessary when $\frac{\rho_{5}}{T_{8}{ }^{3}} \geq 0.1$. From equation 5 we see that energy production by the Salpeter process is linearly dependent on $\Gamma_{\text {rad }}$.

\section{B. Previous Experimental Evidence}

From the work of Cook et al. (1957) as well as later experiments (Alburger 1960, Ajzenberg-Selove 1960), the spin and parity of the $7.656-$ MeV level are almost certainly or. Hence direct gamma decay to the ot ground state is strictly forbidden, and has not been observed. Both pair emission to the ground atate and the gamma cascade through the $2+$ first excit ed state have been observed.

Although earlier experiments (Kavanagh 1958, Eccles 1959) had 
only been able to place an upper limit of $0.1 \%$ on the ratio ${ }^{\gamma}{ }_{\gamma} / T_{\alpha}$, a recent experiment by Alburger (1961) gave a positive result. ObservIng $p-\gamma-\gamma$ triple coincidences in the reaction $B^{10}\left(\mathrm{He}^{3}, p\right) \mathrm{C}^{12 *}(\gamma \gamma) \mathrm{C}^{12}$ (see Figure 1) he obtained for the branching ratio of the $3.223-\mathrm{MeV}$ $\gamma$-ray to the first state:

$$
T_{3.2 \gamma} / T_{\alpha}=(3.3 \pm 0.9) \times 10^{-4}
$$

A previous set of experiments (Alburger 1960, Ajzenberg-Selove 1960) gave the branching ratio for pair emission:

$$
T_{e \pm} / T_{\alpha}=(6.6 \pm 2.2) \times 10^{-6}
$$

In addition, an absolute value of $\mathrm{e}^{ \pm}$can be calculated (Oppenheimer 1939) from a knowledge of the EO matrix element obtained (Schiff 1955) from inelastic electron scattering data (Fregeau 1956):

$$
\begin{aligned}
& \text { M. E. }=\left(5 \times 10^{-26}\right) \pm 25 \%, \\
& I_{\text {e }}=\left(5 \frac{1}{2} \pm 3\right) \times 10^{-5} \mathrm{eV} .
\end{aligned}
$$

Combining the ge results gives

$$
\begin{aligned}
& r=(8 \pm 5) \mathrm{eV} . \\
& \Gamma_{\mathrm{rad}}=(0.0027 \pm 0.0018) \mathrm{eV} .
\end{aligned}
$$

The present experiment provides a completely independent measurement of the ratio $\mathrm{Irad}_{\mathrm{rad}} / \mathrm{I}$, but requires an estimate of $\mathrm{I}^{\mathrm{i}}$ in order to give $\Gamma_{\mathrm{rad}}$ itself. At present, equation 9 is the best experimental value of 5 . 
II. EXPERIMENT; APPARATUS

A. Reasons for Uaing $\mathrm{N}^{14}(d, \alpha) \mathrm{C}^{12 *}$; Kinematics

The plan of the experiment was to populate the second state of $c^{12}$ in a nuclear reaction, and to observe carbon lons recolling from the reaction. Since the lifetime of the state is known from the alpha width to be of the order of $10^{-16}$ aeconds, and it takes $1.8 \times 10^{-9} \mathrm{sec}-$ onds for a 2 - MieV $\mathrm{C}^{12}$ to travel $1 \mathrm{~cm}$, the recoils can be detected only if radiative decay has occurred. To distinฐูuish $\mathrm{C}^{12}$ ions from other reaction products, a low-resolution magnetic spectrometer was to be used. Since it vas necessary to impart 28 much momentum as possible to the recoll nucleus, using a $2-\mathrm{MeV}$ Van de Graff accelerator, a reaction with a high $Q$-value and with a heavy (e.g., mass 4) emitted particle was eought. From Figure 1 two reactions are seen to quallfy:

$$
\begin{array}{ll}
\mathrm{c}^{13}\left(\mathrm{He}^{3}, \alpha\right) \mathrm{C}^{12}, & \mathrm{O}_{0}=15.630 \mathrm{MeV}: \\
\mathrm{N}^{14}(\mathrm{~d}, \alpha) \mathrm{C}^{12}, & \mathrm{O}_{0}=13.574 \mathrm{MeV}
\end{array}
$$

The second of these reactions was chosen because a natural nitrogen target could be used; the $\mathrm{C}^{13}$ reaction would require an enriched target. Also, the fact that the energy loss of deuterons in the target would be much less than $F^{3}$ nuclei would allow us to use higher beam intengities for a given target thickness and temperature. Further, the second state of $\mathrm{C}^{12}$ was known to be populated in the $\mathrm{N}^{14}(\mathrm{~d}, \mathrm{Q})$ reaction (Hoyle 1953, Dunbar 1953), and presumably has a higher cross section. 
A schematic diagram of the equipment used is shown in Figure 2. With a deuteron bombarding energy of $1.800 \mathrm{MeV}$, recoil carbon ions at $90^{\circ}$ in the laboratory system heve an energy of $3.6 \mathrm{NeV}$ for ground-state reactions, and $2.5 \mathrm{MeV}$ or $1.7 \mathrm{MeV}$ if the firat or second excited states are formed. The corresponding alpha-particle energies and angles, and also the flight time $t$ of the $c^{12}$ ions through the $85-$ cm path length of the spectrometer, are given in Table I. Recoil lons of the proper momentum and energy were observed in delayed coincidence with alphe particles of the proper energy and angle, the delay being equal to the proper flight time for the ion. The two pulses $\left(c^{12}\right.$ and $\left.a\right)$ were displayed on a dual-beam oscilloscope and photographed.

\section{B. Target Manufacture}

Target requirements for this experiment were stringent. Because of the low yield, it was desirad to have a high $\mathrm{N}^{14}$ content; however the active layer had to be kept thin for the recoil lons. The calculated otopping power of $\mathrm{VN}$ (vanadium nitride) for $1.7-\mathrm{MeV} \mathrm{C}^{12}$ ions is about $400 \times 10^{-15} \mathrm{eV} \mathrm{cm} \mathrm{cm}^{2} /$ molecule; bence the maximum al lowed target thickness for VN would be about $10^{18}$ molecules $/ \mathrm{cm}^{2}$ for a 10\% energy loss in the target. The backing of the target had to be transparent to the emerging alpha particles, yet strong enough to withstand the bearn and the target-rnaking procedure.

Organic materials, such as adenin $\left(\mathrm{C}_{5} \mathrm{~N}_{5} \mathrm{H}_{5}\right)$, although at tractive because of thei $x$ high nitrogen content, had to be discarded because of their inability to withstand high temperatures. 
Among the refractory metallic nitrides, gallium nitride was thought to sublime without decomposing (Gmelin 1936, Sime 1956). However, all attempts at vacuum evaporation at temperatures from $950^{\circ} \mathrm{C}$ to $1400^{\circ} \mathrm{C}$ resulted in decomposition; no nitrogen could be detected on the targets. An attempt to evaporate silicon nitride failed similarly.

Usable VN targets were made by evaporating vanadium onto mounted $C$ foils, and nitriding by heating in an atmosphere of ammonia. An alpha-particle beam was used to heat the target. (See Figure 3.) The process is described in detail in Appendix A. The same procedure was tried with sllicon, but apparently the temperature was not aufficient to cause nitriding. When titaniurs was evaporated onto carbon folls, they invariably broke, but some success was realized with $T_{i}$ by evaporating before the foils were mounted.

Target composition was checked by elastically scattering $1-$ MeV protons from the target at a laboratory angle of $150^{\circ}$. Using a $10 \frac{1}{2}$ inch double-focusing magnetic spectrometer, the protons scattered from different masses in the region of interest were well resolved. Figure 4 shovy the profile of typical target, and Table II lists the compositions of the various targets used during the experiment. Details of the calculation are included in Appendix $A$.

\section{Recoil-Ion Detection}

The alternating-gredient magnetic spectrometer indicated in Figure 2 has been described by Martin and Kraus (1956). The entrance slit of the magnet is elliptical with the major axis vertical. Being 
$5 / 32$ inch wide, 0.963 inch tall, and 3.25 inches from the target, it subtends a solid angle of 0.011 steradians, yet has a mean width in the reaction plane of only $2.4^{\circ}$. The path length through the magnet from target to detector is $85 \mathrm{~cm}$. An image slit $1 / 8$ inch wide was used for most of the experiment. The elastic-acattering edge of protons from a gold foll showed a momentum resolution of $5 \%$ for this slit. This large value of $\Delta p / p$ was very useful to obtain a reasonable counting rate. For calibration, a 1/32-inch olit was also used; the gold scattering indicated a resolution of $1.3 \%$ for this narrower slit.

With the axis of the entrance aperture of the magnet set at a lab angle of $90^{\circ}$, the object position was determined by finding the maximum counting rate with respect to motion of the bearn spot. A $\mathrm{B}^{10}$ target was bombarded with $1.17-\mathrm{MeV}$ protons; $1.37-\mathrm{MeV}$ alpha particles from the reaction $\mathrm{B}^{10}\left(\mathrm{p}, \alpha_{0}\right) \mathrm{Be}^{7}$ were detected at the focus of the magnet, and $431-k e V$ gamma rays from $B^{10}\left(p, \alpha_{1} \gamma\right) B e^{7}$ were used as monitor counts. Beam position (vertical and east-west) could be measured to within 0.003 inch on a quart $z$ diak at the rear of the chamber; north - south motion was measured by turnscrews. Only vertical motion of the beam spot seriously affected the counting rate; a deviation of $1 / 32$ inch either direction from the optimum position reduced the counting rate to one-half. A similar motion in either horizontal direction had only a 15\% effect. A beam spot approximately $1 / 16$ inch high and $1 / 32$ inch wide (1/16 inch square on the target) was used during the experiment. 
The detector at the magnet focus was $7 \times 14-\mathrm{mm}$ gold-silicon surface-barrier detector manufactured by Oak Ridge Technical Enterprises Corporation (ORTEC). Initlally counters made in this laboratory were used, but the commercial unit was installed before any data were obtained.

The pulse from the detector was amplified as shown in Figure 2 by a fast-amplifier system consisting of a single triode (417A) preamplifier and four Hewlett-Packard type 460A wide-band amplifiers. At the input to the second amplifier the pulse was clipped to 40-nsec width with a shorted length of 93-ohm cable. The amplified positive pulse was fed to the coincidence mixer and to the lower-beam input of a Tektronix type 555 dual-beam oscilloscope. The pulse height was about 1 volt for a 3-MeV particle; a vertical deflection of $0.5 \mathrm{~V} / \mathrm{cm}$ was used. On the lower beam in Figure $5 \mathrm{~b}$, the two groups are deuterons (1.13 MeV) and alpha particles (2.27 MeV).

The pulse from the detector was also fed through a 1000-ohm resistor to a low-nolse integrating preamp (ORI EC type 100A). The preamp output wa connected to the internal amplifier of a 400-channel analyzer (Radiation Instruments Development Laboratories type 34-12). These are also shown in Eigure 2.

\section{Alpha Detection}

The target chamber used for the experiment (see Figure 6) has provision for continuous varlation of the angle $\theta$ from $35^{\circ}$ to $90^{\circ}$. Since it was built before solid-state detectors were developed, it was designed for use with a Cal crystal and a photomultiplier extending 
outside the chamber through a slot, with an O-ring as a sliding vacuum seal. In any currently designed chamber, the counter would more logically be mounted completely within the chamber, on a rotating arm concentric with the target rod.

A I ectangular slit is mounted in the counter holder to define the solid angle of the alpha detector. A slit 0.032 inch wide and 0.200 inch high, about the same solid angle as the spectrometer aperture, was used in early runs, but oventual substitution of a slit 0.060 inch wide increased the coincidence counting rate and decreased the dependence on the determination of the angle $\theta$. The effect of this change is discussed in section IV $D$ below.

Gold-silicon surface-barrier detectors were made according to the recipe of Dearnaley and Whitehead (1961). However after commercial counters were obtained for use at the magnet focus, our mounting technique was changed, as shown in Figure 7 , in imitation of the method used by ORTEC. In these units, epoxy is poured up to the level of the front surface of the silicon, and the gold is evaporated over the entire front aurface to make it 8 own contact to the grounded case. The signal lead is made to the rear surface of the silicon with conducting silver paint. The diode characteristics of three counters made in this fashion, using 900 s-cm n-type silicon, are shown in Figure 8. An alpha pectrum from a ThB source, taken with counter $9 F$ and the bissed amplifier as in Figure 2, is shown in Figure 9. Although a resolution of $120 \mathrm{keV}$ is not out standing for solid-state detector, it is quite sufficient for this experiment. 
The fast amplifiers shown in Figure 2 for the alpha channel are similar to those in the $\mathrm{C}^{12}$ channel, with the "variable delay," used to delay the alpha pulse by a time equal to the flight time of the $\mathrm{C}^{12}$ recoil ion, inserted between the firat and second amplifiers. The delay consisted of from 0 to 255 feet of RG $14 / \mathrm{U}$ cable, which could be switch selected in increments of one foot. The signal velocity In this cable is $0.85 \mathrm{c}$; hence each foot of cable corresponds to a delay of 1.2 nsec.

The pulseg from the alpha counter were higher than those from the $C^{12}$ counter because the alpha energy was higher. Therefore less amplification was needed in the alpha channel in order to get the required pulse height for operation of the coincidence mixer. It was found that two of the Hewlett-Packard amplifiers could be replaced with type 460B's, which have lower gain but also have lower noise level. The output pulses were fed to the coincidence mixer and to the upper-beam input of the oscilloscope; the pulse height of an 8.5 MeV alpha particle was 1 volt (see the second highest group in Figure 5b). The scope gain used was $0.5 \mathrm{~V} / \mathrm{cm}$.

In place of a slow amplifiex system such as used in the $\mathrm{C}^{12}$ channel, the alpha channel had a biased amplifier (see Figure 2). The 40-nsec-wide fast pulse was fed through an additional Hewlett-Pacicard type $B$ arsplifier to the biased amplifier which vas set to discriminate against the high background of elastically scattered deuterons. The atretched and amplified pulse was then recorded on an RIDL 100-chansel analyzer, so that gains could be monitored and sceler discriminator 
biase set to count first-state $\left(\alpha_{1}\right)$ and ground-state $\left(\alpha_{0}\right)$ alpha particles.

\section{E. Coincidence Detection}

A tunnel-diode coincidence mixer was used in this experiment (see circuit diagram, Figure 10). Tunnel diodes were also used as diecriminators at esch input of the mixer. Input pulses of the order of half a volt were sufficient to trigger the diodes from the low-voltage to the high-voltage portion of the characteriatic curve. This low-level switching characteriatic was highly desirable because of the 3 mall pulses produced by solid-state counters; any additional gain in the fast $\mathrm{C}^{12}$ channel led to oscillation. The amplifiers included in the mixer produced a $20-V, 2-\mu$ sec pulse for a count in either channel sufficient to switch the tunnel diode. These "fast $c^{1 Z_{1}}$ and "fast $\alpha$ " pulses were counted and recorded as a monitor of the fast-amplifier gains and of the firing levels of the diodes. The coincidence output was a fast-rising pulse uitable for triggering the oscilloscope sweop.

Both the upper and lower beams of the oscilloscope were set to sweep on time base $A$, which was triggered by the coincidence mixer as shown in Figure 2. The sweep time was $0.1 \mu \mathrm{sec} / \mathrm{cm}$, and $\times 5 \mathrm{mag}-$ nification was used on both beams to give a resulting horizontal scale of $20 \mathrm{nsec} / \mathrm{cm}$. During each sweep, a positive $20-\mathrm{V}$ signal is provided at the "' gate out" terminal of the time base unit. This pulse of 1 $\mu s e c$ duration $(0.1 \mu \mathrm{sec} / \mathrm{cm} \times 10 \mathrm{~cm})$ was counted on acaler as the number of coincidences, and was also used as a gate signal for both of the multichannel analyzers. An additional output signal provided 
by the oscilloscope is a "delayed trigger output," which was set to give a pulse at the end of the eweep time. This $8-\mathrm{V}, 0.6-\mu \mathrm{sec}$ pulse was used to trigger the film-advance mechanism of the camera. As an aid to photography the regular P-2 green-phosphor cathode-ray tube was replaced by a CRT with a P-ll blue phosphor. The response of our film was smuch higher in the blue region of the spectrum. Also the P-ll phosphor has a higher brilliance and a much shorter persistence, which is traportant because our camera had no shutter. We were able to photograph persiatent phosphorescence on the green tube for as much as half an hour after turning off an intense beam; no pergistence of a quarter of a second or longer was observed with the P-11 phosphor.

It had originally been planned to observe the pulse-height spectrum from the counter at the magnet focus, gated by the coincidence mixer. However it soon became obvious that most of the $c^{12}$ ions observed were in colncidence with firat-state rather than second-state alphas, and furthemore that separation of $\mathrm{C}^{12}$ from $\mathrm{B}^{11}$ recoils from $C^{13}(d, a) B^{11}$ reactions) would be difficult. (See Figure 2lc.) Thus it was necessary to obtain correlated coincidence spectra, and this was done in two way B. A simple but not always practical method was to write down the addresses from each multichannel analyzer as each coincidence occurred, the difficulty sometimes encountered being the "rapid" counting rate which averaged one count every five seconds. The other method was to photograph the dual-beam oscilloscope and to measure the pulse heights recorded on film. The clead time for this arrangement was only 1 Hec per oscilloscope sweep, since double 
exposures could be recorded while the film was in motion. The film gives an additional piece of information the time separation of the two pulses. This was us oful because it allowed us to decrease the time sensitivity of the coincidence mixer, and yet improve the time resolution of the experiment. Use of a two-dimensional multichannel analyzer, such as has since been ncquired by the laboratory, would not have allowed this time measurement, and would not have had better energy resolution than the film system.

It might have been possible to use an existing camera and Polarold film to take our photographe, but the retall cost of the film used would have been about $\$ 6000$. Furthermore the dead time involved in developing each print and changing film after every eight event would probably have more than doubled the running time of the experiment. Hence it was declded to degign a camera to use film available in 100-foot rolls, and $35-\mathrm{mm}$ film was chosen to obtain the largest practical negative size. Much assiatance in choosing film and lens was received from Mr. Robert Macek and Dr. Hans Kobrak of the Synchrotron Laboratory. Tests with their cameras showed that an extremely fast lens was necesary to record single sweeps at $20 \mathrm{nsec} / \mathrm{cm}$, and that Kodak Linagraph Fan film had botter contrast and speed than either Tri-X or Royal-X Pan films. The fastest lens then available fo: $35-\mathrm{mm}$ cameras, a Nikkor $\$ / 1.1$, wa obtained. The camere design is shown in Figure 11 and discussed more fully in Appendix B. A Beattie-Coleman electric film magazine was used; the circult used to trigger the film-advance motor after each sweep 
of the oscilloscope, shown in Figure 12, is al so described in Appendix $B$, as is the film-developing procedure.

Not being designed for copy work, the lens was corrected only for distances greatex than three foet. Thus when used at six inches, there was considerable barrel distortion, as can be seen in Figure $5 a$. However by using the same lens and the same geometry in an enlargex, all first order distortions were corrected. Figure 5b was printed in this mannex from the same distorted negative se Figure $5 \mathrm{a}$. 
II. PROCEDURE AND RESULTS

A. Non-Coincident Recoil-Ion Spectra

For a magnetic apectrometer, the energy of a particle of mass $M$ and charge $Z$ is given by (Brown 1951):

$$
\mathrm{I}=c_{\mathrm{m}} \frac{\mathrm{z}^{2}}{\mathrm{MI}}
$$

where $C_{m}$ is a constant depending on the radius of the equilibrium orbit through the spectrometer, and $I$ is the current reading of a torsion-balance fluxmeter (Lauritsen 1948). Thus an energy spectrum of the particles accepted by the magnet at a given curseat will show separate groups with different values of $Z^{2} / M$. Figure 13 is such a spectrum, taken with the alternating-gradient opectrometer at a setting $1 / I^{2}=16.5$, corresponding to a proton energy of $1.85 \mathrm{MeV}$. In this spectrum two of the groups are recoll cerbon ions: those with charge (4t) result from first-state events, and the higher energy (5+) group comes from ground-state event 8 . Identification of the groups is aided by taking such spectra over a range of momentum (magnet) settings. Figure 14 is a contour plot of the energy spectra taken over the range $10.7 \leqslant 1 / I^{2} \leqslant 58.8$; counts are plotted $y s$. E and $1 / I^{2}$. The 1/32-inch slit was used at the magnet image, and each spectrum was normalized to 20,000 ground-plus first-state alpha particles counted in the chamber detector.

B. Non-Coincident Alpha Spectra

The alpha-detection system was calibrated with a ThB source 
(see Figure 9). A typical non-coincident alpha apectrum is shown in Figure 15. The four strong alpha groups above $5 \mathrm{MeV}$ (bias is about $4 \mathrm{MeV}$ for Figure 15) are identified by their energies as the three lowest states of the reaction $\mathrm{N}^{14}(\mathrm{~d}, \alpha) \mathrm{c}^{12}, \alpha_{0}, \alpha_{1}$, and $a_{2}$ respectively. and the ground atate of $C^{13}(d, \alpha) B^{11}$. All the groups must be alpha particles because the counter blas was such that protons of $4 \mathrm{MeV}$ or more would penetrate the depletion layer, resulting in pulses below the bias of the amplifier.

\section{Procedure for Recording Coincidences}

After positive identification of the various particle groups was made on the basis of the non-coincident spectra, the experimental procedure was a follows. First, the spectrometer current was varied and recoil-ion energy spectra were taken to find the maximum non-coincident counting rate of $\mathrm{C}^{12}(4+)$ recoils from first-excitedstate eventi. (See Figure 16. This figure and all others referred to in paragraphs $C, D$, and $E$ of this section refer to that portion of the dats which was taken on film 15.$)$ All counting rates are normalized to the number $\left(\alpha_{1}+\alpha_{0}\right)$ of first-state and ground-state alphas observed in the chamber counter, with the angle $\theta$ set to approximately the proper value. Then without changing the magnet current, the delay was adjusted so that coincident pulses observed on the dual-beam oscilloscope occurred at the same time; Figure 17 is a multiple exposure showing five $C^{12}(4 t)-q_{1}$ coincidences. Then the angle $\theta$ was varied to find the maximum coincidence counting rate for $c^{12}(4+)-q_{1}$ events (see Figure 18). The maximum rate per $10,000\left(\alpha_{1}+\alpha_{0}\right)$ is 
called $R_{1}$.

After thus optimizing, the three parameter were changed to the appropriate values for $c^{12}(3 \div)-\alpha_{2}$ events.

$$
\begin{array}{ll}
\text { Fluxmeter: } & I_{2}=I_{1} \times \sqrt{\frac{2.511}{1.706}} \times \frac{3}{4}=0.910 I_{1} \\
\text { Delay: } & \varepsilon_{2}=l_{1}+24 \text { feet } \\
\text { Angle: } & \theta_{2}=\theta_{1}-3.7^{\circ}
\end{array}
$$

The camera was connected, and runs of $40 \mu f$ on the charge integrator (or about $375 \mu$ coulomb of beam) were made. As each coincidence occurred, the buzzer wired in parallel with the film-advance motor sounded, and an attempt was made to record the addresses from the 400-channel analyzer (recoil ion) and the 100-channel analyzer (biased alpha spectrum) on a line with the frame number of the picture. These pulse heights were used to supplement the information on the film, and also served as a continuous check on gains and the overall performance of the system. The number of coincidences counted (number of sweeps of the oscilloscope) was also noted, in order to keep track of double and multiple exposures. Each sweep war recorded clearly even if the film was in motion; hence the dead time of the system was not determined by the half-second film-advance cime, but only by the $1-\mu \sec$ sweep time of the oscilloscope. At the end of exch charge integration, the following numbers were recorded: elapsed time, number $\left(\alpha_{1}+\alpha_{0}\right)$ of alphas of first-state energy and above (channel 30 and above in Figure 15), number $\alpha_{0}$ of ground-state and higher alphas (channel 70 in Figure 15), number "fast $\alpha$ " of alpha singles sufficient to trigger the coincidence mixer, and number "fast $c^{12, "}$ 
of particles in the recoll-ion channel energetic enough to trigger a coincidence. Thus a continuous check on the triggering levels for the coincidence mixer was made, as well as a check on target deterioration. Occasional photographs (such as Figure 5) of non-coincident spectra were taken as a check of gaing and scope level shifts.

The results of a run are contained in three numbers: $R_{1}=c^{12}(4+)-\alpha_{1}$ coincidence rate per $10,000\left(\alpha_{1}+\alpha_{0}\right)$; $\alpha=\left(\alpha_{1}+\alpha_{0}\right) / 10,000$;

Counts $=$ number of $c^{12}(3+)-\alpha_{2}$ events observed.

\section{Energy and Time Normalisation}

After films were developed, they were projected onto graph paper using the camera lens, and forming an image the same size as the original scope display. Each pulse height was estimated to 0.01 $\mathrm{cm}$ (probable error $\pm 0.03 \mathrm{~cm}$ ), and the horizontal separation of the centroids of the two pulses was estimated to $0.1 \mathrm{~cm}$ (probable exror $\pm 0.2 \mathrm{~cm}$ ). The graph paper was adjusted so that the base line of the upper beam (alpha pulses) was on zero; correction was made in the normalization for the non-zero base line of the lower beam. At this stage noise and peculiar pulse shapes were rejected. Double exposures were also examined at this time; it was generally possible to correlate them properly by position on the film and by comparing pulse heights to observed multichannel analyzer addresses, if any, but occasionally two events were so overlapped that it was impossible to decide which upper-beam pulse belonged to which lower-beam pulse. These cases were discarded; in no case was a possible "real" $c^{12}(3+)-\alpha_{2}$ 
event involved. For identification purposes, the second event of a double exposure was given the negative of the frame number on which it occurred; all other events were identified by frame number. Of the 896 frames exposed on film \#15, 45 were non-coincident calibrations, 11 extra swoeps were recorded from double exposures, and 349 frame s were noise; thus 513 coincidences were recorded on film \#15.

For each event, an IBM card was punched with the following six numbers: film and frame number, 100-channel address (alpha) if avallable, 400-channel address (recoll ion) if available, upperbeam pulse height (alpha) in $\mathrm{cm}$. lower-beam pulse height (recoll ion) in $\mathrm{cm}$, and horizontal separation (positive if lower beam displaced toward the right) in $\mathrm{cm}$. The left half of Table III is an example showing the above information a punched on cards for frames 320 through 416 of film ti5. Where no entry was punched for an analyser address, an entry "-0." appears in Table III.

Since gains were adjusted each day, and subject to variation in any one day, the data were normalized in groups. The punched cards were fed to an IBM 7090 electrontc data-processing machine in groups of between 30 and 300 , depending on gain shifts or oscilloscope level shifts. The strongest coincident group was first-state alpha particles in coincidence with $C^{12}(3+)$ ions which had lost sufficient energy by atraggling in the target to come through the magnet with the energy and velocity appropriate to second-state events. Because it was strongest, this group was used for calibration of all 
thitee variables: both energies and the time. An estimate of the st ${ }_{1}^{-}$ pulse height on the upper beam vas made, and the computer aroraged all pulse heights within $10 \%$ of the estimate and set the reault equal to $3.52 \mathrm{hieV}$. Asauming the oscilloscope pulse height proportional to energy, all upper-bearn pulse heights were converted into energieg (E UP in Table III). Also, a linear relation between the addreas of tize 100-channel analyzer and the upper-beara pulse height was found by leagt aquares, and the addresses were converted into energies (E100 in Table III). If no address was given, the enexgy set equal to E UP, but with a minus sign to indicate that it was not an inclependent measure of the energy.

The recoil-ion energy was normalized in the same manner, with the exception that the 400-channel analyzer address was the pro mary standard when it was available. This was done because of the poor resolution of the lower beam on the film resulting from the higher gain and higher nolse level in the fast $\mathrm{C}^{12}$ channel as coispared to the fast alpha channel. (Compare upper-and lower-beasz resolutions in Figure 17.) Thus the averages of all addresses within $10 \%$ of an estirnated address for the $C^{12}(3+)$ ion was set equal to 1.706 MeV, and the address was assumed proportional to energy. Recollion energies E LOW and E 400 (see Table III) were calculated from the lower beam and from the 400-channel analyzer address when available; again $\mathrm{E} 400=-\mathrm{W}$ LOW if no address was recorded.

The time scale was normalized by averaging all values of the horizontal separation within $0.3 \mathrm{~cm}$ of the estimated separation of 
the $\mathrm{C}^{12}(3+)-\alpha_{1}$ coincidences, and setting this average equal to $\Delta t=0$. The horizontal scale factor was taken to be the oscilloscope setting of $20 \mathrm{nsec} / \mathrm{cm}$, and a $\Delta t$ was calculated for each event (DT in Table III).

\section{E. Determination of the Number of Counts}

After the normalization was completed, esch event was added into the appropriate location in a $30 \times 27 \times 20$ array representing $E U$, $|\mathrm{E} 400|$, and DT respectively. (The absolute value of E400 was taken because $\$ 400$ had been given negative sign if calculated from the lower beam instead of the 400-channel analyzer.) The range of alpa particle energy was from 4.625 to $12.125 \mathrm{~N} / \mathrm{VV}$ in $0.250 \mathrm{NeV}$ steps; the range of recoil-ion energy was from 0.875 to $4.250 \mathrm{MeV}$ in 0.125 MeV steps; and the range of $\Delta t$ was from -52.5 to $+47.5 \mathrm{nsec}$ in 5.0 nsec steps, where $\Delta t=0$ corresponds to a flight time of 162 neec, and a negative $\Delta t$ indicates a higher velocity ion. The three-dirensional array was printed out as 20 separate $27 \times 30$ two-dimenaional arrays, each corresponding to a different value of $\Delta t$. The three center sections (At $=-5,0$, and $+5 \mathrm{nsec})$ for the data from film 115 are reproduced as Figure $19 \mathrm{a}, \mathrm{b}$, and $\mathrm{c}$. The circled group is the $c^{12}(3+)-q_{2}$ group, and contains 26 counts. The entire three-cimensional array is plotted in isometric projection a a color-coded correlated coincidence spectrum in Figure 20. * Color is used to identify the vertical (time) axis, and a fourth dimension is provided by the area of the

* It is not practical to provide copies of Figure 20 in color with reproductions of the thesis. However either a duplicate $2 \times 2$-inch color slide or an $8 \times 10$-inch color print can be obtained at cost from the Kellogg Radiation Laboratory. In 1962, the cost of a duplicate slide was 30 c and the cost of a print was $\$ 3.50$. 
circle at each point, the area being proportional to the number of counts. The figure show the $\mathrm{c}^{12}(3+)-\alpha_{2}$ group well separated from all other groups, at $E_{Q}=6.1 \mathrm{MeV}, E_{C_{12}}=1.7 \mathrm{MeV}$, and $\Delta t=0 \mathrm{nsec}$.

The vertical columins aloug the left rear wall of the figure are deuterons in accidental coinciderce with the various alpha-particle groups. For the $\alpha_{0}$ and $\alpha_{1}$ groups, they extend through all timo from -40 to $+40 \mathrm{nsec}$ for the less energetic alphas with graller pulse heights, only deuterons within about $20 \mathrm{nsec}$ registered as coincidences. Two other groups besides the group of interest occur at $\Delta t=0$; the $c^{12}(3+)-G_{1}$ group used for calibration, and also the $C^{13}(d, a) B^{11}$ ground-state group, which is the closest neighbor to the group of interest (see Table I). 'At $\Delta t=-40$ nsec, higher energy $C^{12}(4 t)$ ions straggled from ground-state events appear in coincidence with ground-state alphas. The remaining Broup at $\Delta t=-30 \mathrm{nsec}$ has mass 12 (from energy and velocity deterrainations), but an apparent charge of $3 \frac{1}{2}$ l This can be explained by recalling that first-excited-state events are $10^{4}$ times as numerous as secondstate events, and noting that the energies for the group are approximately thcse of the particles from a first-state reaction. Evidently the structure of the magnet is such that a $(4+)$ ion can reach the ceater of the magnet, and if it undergुoes a charge-exchanging collision there it may continue through the rsagnet as a $(3+)$ ion. A collision probability of 1 in $10^{4}$ within perhaps $4 \mathrm{~cm}$ of path length between the two segments of the magnet would be sufficient to produce the observed group. At a pressure of $10^{-6} \mathrm{~mm} \mathrm{hig,} \mathrm{this} \mathrm{would} \mathrm{correspond} \mathrm{to} \mathrm{a} \mathrm{collision} \mathrm{radius} \mathrm{of} 1.5 \AA$. To show that the visualization of the coincidence counting rate 
as a function of three variables is necessary to identify counts, the data of film \#15 are also presented as functions of the single variables $\Delta t, E_{\alpha^{\prime}}$ and $E_{C^{12}}$ (see Figure 21 ). In each of this figure the solid areas are the 26 real counts identified from Figures 19 and 20 , and the shaded areas are the accidental coincidences with elastically scattered deuterons in the recoil-ion channel. In Figure $21 a$ these randorn coincidences provide a measure of the resolving time of the coincidence mixer. In Figure $21 \mathrm{~b}$ the non-coincident alpha spectrum is reproduced (cf. Figure 15) by the accidental deuteron coincidences. Figure 2lc confirms the statement made earlier that it is impossible to identify count $s$ by observation of the coincident recoil-ion spectrum alone.

\section{F. Experimental Results}

After approximately 61 hours of running time with an average beam intensity of $1 \mu \mathrm{A}$, the total number of counts obtained was 170 . The total of non-coincident $\alpha_{1}$ and $\alpha_{0}$ was $4.01 \times 10^{7}$. The value of $R_{1}$, the $C^{12}(4+)-q_{1}$ coincidence rate which was used as a measure of efficiency, varied with changes in target thickness, slit geometry, and possibly with vertical beam position; an approximate mean value for $R_{1}$ is 380 , compared to the rate of $0.042 c^{12}(3 t)-c_{2}$ coincidences per $10,000\left(\alpha_{1}+\alpha_{0}\right)$. A sumrary of the data for individual runs is given in Table IV.

A background run was made using target C7-XIV, which had not been nitrided. It did contain about $0.05 \times 10^{17} \mathrm{~N}^{14}$ atoms $/ \operatorname{csm}^{2}$, how ever, which were probably included during the evaporation of vanadium, which is a good getter. The run lasted 5 hours (at 1- $\mu$ A beam intensity), 
which would have been sufficient to obtain 15 counts on an average target, or 0.2 counts from the nitrogen in the target used. Fo $\mathrm{C}^{12}(3+)-q_{2}$ counts were observed. The values of $R_{1}$ and $\alpha$ are included in Table IV. 
IV. ANALYSLS OF RESULTS

\section{A. Equation for $1 \mathrm{rad} / \mathrm{I}$}

The ratio $\Gamma_{\mathrm{rad}} \mathrm{I}$ is the ratio of the number of $\mathrm{C}^{12}$ surviving to the number of $\mathrm{C}^{12 *}$ formed in the second excited state, or to the total number of $\alpha_{2}$ prodiced in the reaction. If $\epsilon_{2}$ is the efficiency of the system for detecting second-state coincidences,

$$
\frac{I_{\text {rad }}}{L}=\frac{\text { observed } c^{12}(3+)-\alpha_{2} \text { coincidences }}{\epsilon_{2} \times \operatorname{total} \alpha_{2}} .
$$

The efficiency $\epsilon_{1}$ for detecting first-state coincidences can be found directly by experiment, since for the first state we know that $\left(I_{\mathrm{rad}} / \mathcal{L}\right)_{1} \equiv 1$; hence only the relative efficiency $\epsilon_{2} / \epsilon_{1}$ must be cal culated.

$$
\begin{aligned}
& \epsilon_{1}=\frac{\text { observed } c^{12}(4+)-q_{1} \text { coincidences }}{\operatorname{total} Q_{1}} \\
& \frac{L_{\text {rad }}}{E}=\frac{\text { Counts } / Q}{R_{1}} \times \frac{\epsilon_{1}}{\epsilon_{2}} \times \frac{q_{1}}{Q_{2}} .
\end{aligned}
$$

Besides the relative population factor $P=\alpha_{1} / \alpha_{2}$ included explicitly in equation 14, the relative efficloncy includes three correction factor 3 . The rzost obvious, C, concerns the ratios of the various ionic-charge states of the recoiling nucled, since we observe only a single charge state at a time. The second, $G$, has to do with differences in the effective golid angle for the two states, including the effects of deflectione of the recoiling nuclei by gamma emisgion. The third correction. $T$, has to do with the momentum width 3 of the recoil-ton groups from the two states, corspared to the $5 \%$ momentum window of the spectrometer. 


$$
\begin{aligned}
\epsilon_{2} / \epsilon_{1}= & C \times G \times T \\
= & \frac{C^{12}(3+) / C^{12}(\text { total }) \text { at } 1.71 \mathrm{MeV}}{C^{12}(4+) / C^{12}(\text { total }) \text { at } 2.51 \mathrm{MeV}} \times \\
& \times \frac{\text { effective d } \Omega \text { for second state }}{\text { effective d } \text { for first state }} \times \\
& \times \frac{\% \text { of } 2^{\text {nd }} \text { atate } C^{12} \text { group in momentum window }}{\% \text { of } 1^{8 t} \text { state } C^{12} \text { group in momentum vindow }}
\end{aligned}
$$

Then

$$
\frac{1 \mathrm{rad}}{L}=\frac{\text { Counts }}{d R_{1}} \times \frac{P}{C G T} .
$$

or $\frac{T r a d}{I}=\frac{\text { Counts }}{W}$, where $w=\frac{C G T}{F} \times \alpha R_{1}$.

Now the number $W$ is proportional to the number of counts which would be obtained if there were no statiatical fluctuations; thus the par cent statiatical exror for a given run is proportional to $1 / \sqrt{W}$. If the result of each run is to be weighted inversely as the square of the error, the proper weighting factor is W. But

$$
W \times I_{\mathrm{rad}} / I=\text { Counts: }
$$

hence

$$
\left(\frac{I \mathrm{rad}}{I}\right)_{\mathrm{avg}}=\frac{\Sigma(\text { Counts })}{\Sigma(M)}
$$

Calculation of the various factors appearing in equation 16 and finally the calculation of $\Gamma_{\mathrm{rad}} / \Gamma$ are given in the following sections. 


\section{B. Population Factor, P}

From the alpha-particle spectrum of Figure 15, taken at the bombarding energy $E_{d}=1.800 \mathrm{MeV}$ and the angle $\theta=67^{\circ}$ used in the experiment, the factor $P$ is

$$
P=\alpha_{1} / \alpha_{2}=3.00 \pm 0.09
$$

2 was measured at several energies and angles, and was found to be nearly constant between 1.7 and $1.9 \mathrm{MeV}$ and between $67^{\circ}$ and $71^{\circ}$. It is interesting to compare this result (equation 18 ) with the value $P=17$ obtained by Hoyle (1953) and Dunbar (1953) at a bombarding energy of $672 \mathrm{keV}$ and at a lab angle of $90^{\circ}$.

\section{Charge-State Factor, C}

To determine the charge-state ratios of carbon lons emerging from one of our VN targets, an elastic-scattering experiment was doize. Alpha particled from the Office-of-Naval-Research tandem accelerator in Sloan Laboratory were used to bormberd the carion-foll backing of target C5-II (Table II), and $\mathrm{C}^{12}$ ions from $\mathrm{C}^{12}\left(\mathrm{He}^{4}, \mathrm{C}^{12}\right) \mathrm{He}^{4}$ were observed ernerging from the $V N$ side of the target at a laboratory angle of $35^{1}{ }^{\circ}$. The recently installed 24 -inch double-focusing magnetic spectrometer (Groce 1962) was used to analyze the emerging beam, and was varied to observe as many charge states as posotble at each of three bombarding energies. The maximum solld angle $(\approx 0.014$ steradian $)$ of the magnet was used, and the image slit was $\frac{1}{4}$ inch wide. The detector was a surface-barrier counter, and the energy spectra were recorded on a 400-channel analyzer. A single-channel discriminatoz 
was set to include the $\mathrm{C}^{12}$ peak, which occurred at the same energy for each setting of the magnet. Another solid-state detector within the target chamber was set at about $90^{\circ}$ and the elastically scattered alphas were used as a monitor of the charge integration and target deterioration.

At each of the three energies, which coszesponded to froundstate, first-state, and second-state recoll energies from the $N^{14}(\dot{a}, \alpha) c^{12}$ reaction, the magnet was set at the appropriate value to observe a prominent charge group, and the bombarding energy was varied to find the maximum counting rate. At the angle chosen, the $c^{12}$ recoil has half of the energy of the incident particle; however because the ions pass through about half the carbon backing and all of the VN layer, the bombarding energies (given in Table V) had to be conaiderably higher. At each bombarding energy the magnet current was changed in proportion to $1 / Z^{2}$ to observe the charge states $Z$.

Shown in Table $V$ are the experimental regults in the form of the $c^{12}$-counting rate divided by the alpha-counting rate in the chamber counter. Three or more readings were taken at each charge; the errors quoted in the table are either the spread of the readings or the gtatiotical error, whichever was larger.

The data from Table $V$ are converted into ratios in Tabls VI. The quoted errors were calculated as follows. Let the ratio corresponding to a charge state whose counting rate vas $A$ be

$$
\mathbf{R}=\frac{\hat{A}}{A+B} \text {. }
$$


where B represent the sum of all other charge states besidea A. Then the square of the exror in $R$ is

$$
\begin{aligned}
(6 R)^{2} & =\left(\frac{\partial R}{\partial A} 6 A\right)^{2}+\left(\frac{\partial R}{\partial B} 6 B\right)^{2} \\
& =\left(\frac{1}{A+B} \delta A-\frac{A}{(A+B)^{2}} 8 A\right)^{2}+\left(-\frac{A}{(A+B)^{2}} 8 B\right)^{2} \\
& =\left(R \frac{6 A}{A}-R^{2} \frac{6 A}{A}\right)^{2}+\left(R \frac{B}{A+B} \frac{6 B}{B}\right)^{2}: \\
\left(\frac{6 R}{R}\right)^{2} & =(1-R)^{2}\left[\left(\frac{6 A}{A}\right)^{2}+\left(\frac{6 B}{B}\right)^{2}\right] .
\end{aligned}
$$

It should be noted that the exrors quoted in Table VI are purely statis tical and do not include estimates of systematic error. Repetition of the experiment under more adverse conditions (instability of the magnetic-field-measuring device and rapid deterioration of the target) indicated that the error should be doubled. The charge ratios from Table VI are plotted in Figure 22 as a function of the velocity of the ions.

The charge-ratio factor $C$ is a ratio of two ratios. Though it is hoped that systematic errors should be reduced by taking ratios, the quoted errors in Table VI are doubled.

$$
\begin{aligned}
& C=\frac{c^{12}(3+) / c^{12}(\text { total }) \text { at } 1.71 \mathrm{MeV}}{c^{12}(4+) / c^{12}(\text { total }) \text { at } 2.51 \mathrm{MeV}} \\
& C=\frac{0.485 \pm 0.007}{0.341 \pm 0.007} \\
& C=1.421 \pm 2.5 \%
\end{aligned}
$$




\section{Geometry Factor, G}

The initial direction of a recoiling $C^{12 *}$ nucleus may be changed by gamma-ray emiagion. A first-state recoil at $90^{\circ}$ has an energy of 2. $511 \mathrm{MeV}$ and a momenturn of $237 \mathrm{MeV} / \mathrm{c}$; emisgion of the $4.433-\mathrm{MeV}$ $\gamma$-ray may deflect it by as much as $1.07^{\circ}$. Similarly the momentum of a second-state recoll is $195 \mathrm{MeV} / \mathrm{c}$, and emisaion of two $\mathrm{y}$-rays with a total energy of $7.656 \mathrm{MieV}$ will deflect the nucleus $2.25^{\circ}$ if both $\mathrm{y}$-rays are in the same direction and at right angles to the path of the ion. In general, as many recoil lons will be deflected into the magnet entrance as are deflected away from it. However since the alpha-particle direction depends only on the initial direction of the recoil ion, an ion deflected into the magnet aperture may not be in coincidence with an observed alphe particle. The losses will be greater for the socond state because of the greater possible deflection, and hence the relative efficiency will be affected.

Since both the spectrometer-entrance blit and the alpha-counter slit were tall compared to their widths, and the alpha slit was at least $2.8^{\circ}$ taller than the spectrometer aperture, only the horizontal component of the deflection was considered. Figure 2.3 shows the notation used in the calculations. The center of the rectangular alpha slit, $R$, is at the angle $\theta_{0}$ corresponding kinematically to $\mathrm{C}^{12 *}$ recoila at $90^{\circ}$, and the angle $\theta$ is the angle determined by kinematics to correspond to a $\mathrm{c}^{12 *}$ ion with an initial dixection different from $90^{\circ}$ by an amount 8. Nomentum $\vec{p}_{\gamma}$ is carried off by the emitted gamma or gammas, and the resulting deflection of the recoil ion is $\psi$, measured in the 
horisontal plane. For small angles, the angle $\psi$ is proportional to the component $p_{z}$ of $\vec{p}_{Y}$ parallel to the beam axis. Thus knowled 0 of the probability distribution for values of $\mathrm{P}_{z}$ determines the probablity distribution for $\psi$.

The derivations of the probability functions ${ }_{1}(\psi)$ for the first state and $W_{2}(\psi)$ for the second state are given in Appendix $C$. If the emisaion of the 4. 433-MeV $\gamma$-ray from the first state is isotropic. which we assumed to be the case, then the distribution ${ }_{1}{ }_{1}(\psi)$ is rectangular (see Figure 24). The situation for the second state is more complicated. The first $\gamma$-ray, $3.223 \mathrm{NieV}$, is isotropic, but there is a strong angular corrolation between the two E2 Y-rays. (From Figuxe 1 the level scheme is $0+, 2+, 0+$.$) If \Theta$ is the angle betreen the two y-rays (Frauenfelder 1955),

$$
W(\cos \Theta)=5 / 8\left(1-3 \cos ^{2} \Theta+4 \cos ^{4} \Theta\right)
$$

The $z$-component of the vector sum of the two $\gamma$-rays must be considered; the magnitude of the momentum $\vec{p}_{\gamma}$ will vary between the sum $(7.656 \mathrm{MeV} / \mathrm{c})$ and the difference $(1.210 \mathrm{MeV} / \mathrm{c})$ of the two garnmas with a probability distribution derived from equation 21 . The final rosult for ${ }_{2}(\psi)$ is shown in Figure 24. Note that both curves in Figure 24 were calculated with $6=0$; there will be a slight variation with 6 because the initial morzentum of the recoil ion is a function of 6 .

The probability of detection as a function of $\delta$ and $\psi$ depends upon the geometrical oponings of the two slits, $R(\theta)$ for the zectangular slit and $E(6, \psi)$ for the elliptical aperture of the magnet. Since the 
rectangular alpha slit is not located on the equator of the apherical co-ordinate system defined by the beam axis, its projection on the aurface of the sphere is curved. The shape of a rectangle projected onto a sphere is derived in general in Appendix $D$, and drawn for two positions of the same slit in Figure 25. The pertinent value of $\theta_{0}$ is $67.18^{\circ}$; the shape at $10^{\circ}$ is included only for contrast. Fortunately the elliptical slit is on the equator; its shape is given by the curve marked "ground state" in Figure 26.

Neglecting the vertical component of $\vec{p}_{\gamma}$, the recoil $c^{12}$ and the e-particle are at the same inclination $\phi$ from the horizontal plane. Hence the minor of the two functions $R$ and $E$,

$$
\underline{w}(\delta, \psi)=\min \{R[\theta(\delta)], E(\delta, \psi)\}
$$

determines the probability that both the alpha and the $\mathrm{C}^{12}$ will be detected. (Account was also taken of the fact that the opening represented by the function $R(\theta)$ was not necessarily at $\phi=0$.) The total effective solid angle for coincidence detection is

$$
d \Omega=\int d \delta \int W(\psi) \Phi(8, \psi) d \psi
$$

This expression was integrated numerically with an IBM 7090 computex for several different rectangular slits and for the two angular probability function $3 w_{1}(\psi)$ and $w_{2}(\psi)$ given in Appendix $C$. The integral $I(\delta)=\int W(\psi) \Phi(6, \psi) d \psi$ for the slit 0.060 inch wide and 0.200 inch high is shown in Figure 26 for the first state and the second state. The curve for the ground state is just $\Phi(8,0)=\Sigma(8)$. The asymmetry of 
the second-gtate curve at $-3^{\circ}$ is due to the edge of the rectangular slit. The results of dS for the several cases are given in Table VII.

The geometrical correction factor $G$ is the ratto of $d \delta^{\circ}$ for the second state to $d f$ for the firat state. The probable error in the calculation of $G$ was estimated on the assurnption that the probable error of each calculated dif it $10 \%$ of the difference between $d \Omega$ and the maximum value, 0.011 steradian. Values of $G$ are also given in Table VII.

\section{E. Thickness Factor, T}

If all of the $C^{12}$ ions entering the magnet aperture have momenta within the $5 \%$ resolution widh of the magnet, they would presumably all be counted. As soon as the momentum vithth of the $\mathrm{C}^{12}$ group exceeds $5 \%$, a correction must be applied to the counting efficiency. There is also a correction to the relative efficiency since the wiath of the group is a function of energy.

The first cause of width is target thickness. Using the mean square charge for $\mathrm{C}^{12}$ ions from Table $\mathrm{VI}$, and proton stopping power data of Bader (1956) for $V$ and Reynolds (1953) for $N$, the energy loss of $\mathrm{C}^{12}$ ions at the energies of interest in $\mathrm{V} N$ cannibe calculated from the relation that $d E / d x$ will be $z^{2}$ times $d E / d x$ for protons of the same velocity. This 13 clone in Table VII. Also in Table VIII are the calculated energy losses at each of the three lon energies for the various targets used. This calculation was done assuming a VN layer with the number of molecules in it equal to the nitrogen content of the target as listed in Table II. The target is inclined at $60^{\circ}$ to the 
incident beam, so the number is divided by cos $30^{\circ}$.

Another source of momentum width is kinematic or dE/d $\theta$ broadening, including the extra width allowed by deflection of recoil Ions into the magnet aperture by $\gamma$-emission. For the full width at half maximum of the energy spread from this cause, it is only necessary to measure the width of the curves of Figure 26 . The values of $\Delta 6$ and the corresponding $\Delta \mathrm{E}_{\delta}$ are entered in Table VIII. Actually the distributions in Figure 26 are functions of the rectangular slit used, but the full width at half maximum is virtually unaffected.

The final wource of momentum broadening considered is the longitudinal component of the momentum of the emitted gamma rays. The probebility distribution for a single component of the gamma momentum has already been calculated and is represented by the distributions $W(\psi)$ in Figure 24. The angle $\psi$ (In radians) is the ratio of $p_{z} / p ; p_{z}$ could just as well be the longitudinal component, and then $\Delta \mathrm{E}_{\gamma} / \mathrm{E}=2 \Delta \mathrm{p} / \mathrm{p}=2 \psi$. The full width at half maximum ou and the corresponding $\Delta E_{\gamma}$ are also given in Table VIr.

The three energy spreads are summed in a simple fashion by taking the square root of the sum of the squares of the full width at half maximum listed in Table VII. The results, expressed as per cent momentum widths, are listed in Table IX along with a few experimental measurements. The spectrometer resolution used to measure widths was $1.3 \%$ in momentum; see for instance Figure 16 which is a measurement of target C6-I, and Figure 14 for target C4-XI. It would not seem unreasoneble to assign a probable error of $15 \%$ to the 
calculated momentum widths.

The factor $T$ required for the relative efficlency is the ratio of the per cent of the second-state group which is within the $5 \%$ momentum window of the spectrometer to the per cent of the first-state group within the window. These percentages are again calculated in a simple manner by assuming the group profile to be square with a width given by Table IX. Results and estimated errors for $T$ are given in Table $\mathrm{X}$. The error of the ratio is taken to be $25 \%$ of the amount of the correction, $1-\mathrm{T}$, combined with an additional 5\% estimated systematic error, from comparing different calculations.

\section{F. Calculation of $\mathrm{r}_{\mathrm{rad}} / \mathrm{T}$}

We now have measured or calculated all factors in equation 16. In Table XI the weighting factors $W$ are calculated for each of the individual runs listed in Table IV. The data for each target are then treated as separate samples, and the values of $I_{\mathrm{rad}} / \Gamma$ for each axe calculated in Table XII and plotted in Figure 27. The mean value of $I_{\mathrm{rad}} / \mathrm{I}$ is also calculated, from equation 17.

The internal error is calculated from the statistical error of 170 counts and the standard deviation of $\Sigma w$, and the external error from the deviations of the five data groups from the mean. Combining the external error with an estimated $5 \%$ systematic error in the quantity $T$, the result of the experiment is

$$
\frac{\Gamma_{\mathrm{rad}}}{\Gamma}=(2.82 \pm 0.26) \times 10^{-4}
$$




\section{v. CONCLUSION}

The result of this experiment is not significantly different from that of Alburger (1961), given in equation 6. Averaging our value with Alburger's, we obtain

$$
\frac{\mathrm{rad}}{i}=(2.9 \pm 0.3) \times 10^{-4}
$$

Using the value for $q$ diecussed in section $I B, Y=(8 \pm 5)$ eV, we obtain

$$
I_{\mathrm{rad}}=(0.0024 \pm 0.0015) \mathrm{eV}
$$

This result is only a factor of $2 \frac{1}{2}$ higher than that used by Salpeter (1957) in his energy-production-rate calculations. With our value for Irad" equation 5 becomes

$$
\epsilon=3.3 \times 10^{21} \frac{P_{5}{ }^{2} x_{\alpha}{ }^{3}}{T_{8}{ }^{3}} f \exp \left(-\frac{43.2}{T_{8}}\right) \mathrm{erg} / 8 \mathrm{sec},
$$

and the reaction rate for the formation of $\mathrm{C}^{12}$ is

$$
P=2.8 \times 10^{26} \frac{P_{5}{ }^{2} x_{0}{ }^{3}}{T_{8}{ }^{3}} f \exp \left(-\frac{43.2}{T_{8}}\right) \text { reactions } / \mathrm{g} \mathrm{sec},
$$

where $P_{5}$ is density in $10^{5} \mathrm{~g} / \mathrm{cm}^{3}, x_{\alpha}$ is the mass fraction of $\mathrm{He}^{4}, T_{8}$ is the temperature in $10^{8}{ }^{\circ} \mathrm{K}$, and $f$ is the electron screening correction given approximately by $\left(\mathrm{B}^{2}\right.$ FH 1957)

$$
f \approx \exp \left[0.88\left(\frac{P_{5}}{T_{8}^{3}}\right)^{\frac{1}{2}}\right]
$$

The mean lifetime for an alpha particle is given by 


$$
\frac{1}{\tau_{3 \alpha}\left(\mathrm{He}^{4}\right)}=\frac{3 P}{n_{\alpha}}=5.7 \times 10^{3} \frac{\left(p_{5} x_{\alpha}\right)^{2}}{T_{8}{ }^{3}} f \exp \left(-\frac{43.2}{T_{8}}\right) \mathrm{sec}^{-1}
$$

This function, neglecting $f$, is represented by the curve labeled 3 Hie ${ }^{4} \rightarrow c^{12}$ in Figure 28, calculated for $\rho_{5} x_{\alpha}=1$. For comparison. the lifetime of a $c^{12}$ nucleus in the reaction $C^{12}(\alpha, \gamma) O^{16}$ is also plotted. The latter rate depends on the unknown alpha with of a level at 7.12 or in $\mathrm{O}^{16}$, just $40 \mathrm{keV}$ below the $\mathrm{c}^{12}+\alpha$ threshold. Fowler and Hoyle (1962) estimate $\theta_{\alpha}^{2}=0.78$ or 0.024 , the former value being preferred theoretically and astronomically. With $\theta_{\alpha}^{2}=0.78$, the lifetime ${ }^{\top}{ }_{\alpha}\left(C^{12}\right)$ for a $C^{12}$ nucleus, in years, is given by

$$
\begin{aligned}
\log \tau_{\alpha}\left(C^{12}\right)= & -23.23-\log P_{5} x_{\alpha}+2 \log T_{8} \\
& +\frac{30.08}{T_{8}^{I / 3}}+\frac{0.18}{T_{8}^{2 / 3}} .
\end{aligned}
$$

and if $\theta_{\alpha}^{2}=0.024$ the constant on the right becomes -21.72 . Curves for both values of $\theta_{\alpha}^{2}$ are shown in Figure 28; the solid curve is for $\theta_{\alpha}^{2}=$ 0.78 . An experiment by Larson and Spear now in progress in Sloan and Kellogg Laboratories may determine the $c^{12}(\alpha, \gamma) \mathrm{O}^{16}$ rate more precisely.

Thus the rate of the $3 \alpha$ process as a function of denaity and temperature is now known to about $61 \%$, the major source of uncertainty being the total width of the $7.656-\mathrm{MeV}$ level in $\mathrm{C}^{12}$. In this experiment, the ratio of the electromagnetic decay width to the total width was measured with a $9 \%$ probable errox. In order to improve our knowledge of 
$F_{\text {rad }}$ it would be necessary to increase the accuracy of the determination of $\mathbf{I}$. A repetition of the inelastic-electron-scattering experiment to determine the absolute value of $\Gamma_{\text {et }}$ might be called for. A reduction in the exrox asigned to the EO matrix element from $25 \%$ to $10 \%$ would provide a final result for $\Gamma_{\text {rad }}$ with the error reduced to $40 \%$ If the ratio $I_{e} \pm / \Gamma$ were also known to $20 \%$, the total error in $\Gamma_{\mathrm{rad}}$ would be $30 \%$. 
APPENDIX A

Thin Nitrogen Targets

1. Carbon-foil backing

Microscope slides were cleaned thoroughly and then wet with glycerol. After the glycerol had been allowed to drain for an hour. the slides were placed in a vacuum system. A day was allowed to pump off the glycerol, and then carbon was sputtered onto the slides. To obtain a thick layer of carbon $\left(-40 \mu \mathrm{g} / \mathrm{cm}^{2}\right)$, sputtering was done in four fifteen-minute perfods, with about fifteen minutes for cooling between each.

The carbon on the slides was divided into from ten to sixteen sections with a scribe, and the folls were floated off the slide in a shallow dish of distilled water two at a time. They were then lifted from the surface of the water on tantalum blanks which had a $1 / 4-$ inch or 5/16-inch hole. The foil had to cover the hole, and great care had to be taken to prevent wrinkles. Although it was easier to mount foils on clean blanks, it was found that the folls would adhere more permanently to blanks which had been.lightly greased with vacuum grease.

\section{Vanadium evaporation}

Vanadium in wire or grain form wa evaporated in vacuum from a tungsten boat. Because vanadium is an excellent getter, care was taken to shield the target blanks during the first melting of the vanadium. The pressure in the vacuum system was generally of the order of $10^{-6} \mathrm{~mm} \mathrm{Hg}$. About $20 \%$ of the folls were broken during evaporation. Inatead of air, dry nitrogen gas was admitted to the 
syotem after ovaporation to $t r y$ to prevent oxidation.

\section{Nitridine}

The nitriding procedure was to heat the target with an alpheparticle beam at the straight-through port of the $2-M e V$ Van de Graaff machine. Diatilled ammonia was admitted to the chamber, as shown in Figure 3. The ammonia was first frosen (liquid $\mathrm{N}_{2}$ temperature), and contaminants were pumped off. After boing warmed and frozen three times, there was no change in the thermocouple-gauge roading when the (frozen) ammonia flask was opened to the syatem. Thus any oxygen was removed from the ammonla, but watez was frozen with the ammonia. In order to keep water frosen curing the nitriding, the ammonia flask was cooled to dry ice temperature, where the vapor pressure of $\mathrm{NH}_{3}$ is $43 \mathrm{~mm} \mathrm{Hg}$ and the vapor pressure of $\mathrm{H}_{2} \mathrm{O}$ is 0.00055 mm Hig.

The pressure of $\mathrm{NH}_{3}$ in the chamber during nitriding was of the order of $1 \mathrm{~mm} \mathrm{Hg}$. With the differential pumping and cold trap arrangement ahown in Figure 3 , no change was noted in the pressure in the accelerator column.

A beam of alpha particles (typically $0.8 \mu \mathrm{A}$ at $700 \mathrm{keV}$ ) was used to heat the target to red heat, temperature being controlled by the needle valve controlling the ammonis pressure. The only analysis of the beam energy was done by the mas-separating magnet, but that deflected the beam enough to provide a signal for regulating the energy. The target was moved so that the entire central region was heated; ten minutes total nitriding time seemed to bo sufficient to nitride a 
target as completely as possible. About $20 \%$ of the foils were broken during nitriding.

\section{Composition}

Protons of $1 \mathrm{MeV}$ were scattered from each target at the electrostatic-analyzer station of the $2-\mathrm{MeV}$ Van de Graaff. Scattered particles were observed at a laboratory angle of $150^{\circ}$ with the 10t-inch double-focusing spectrometer. Using the equation derived by Snyder (1950) for yleld from a thin target in such a spectrometer, we have

$$
\frac{n t}{\cos \theta_{1}}=\frac{2 R}{q} \times 10^{15} \int \frac{N_{q}(I)}{I} a \text {, }
$$

where

nt $=$ thickness of target in nuclei $/ \mathrm{cm}^{2}$

$\theta_{1}=$ angle between beam and target normal

$R_{c}=$ resolution determined by slits at collector

$\sigma=$ cross section of reaction $=4 \pi \frac{d \sigma}{d \Omega}$

$q=$ charge deposited

$\Omega=$ solid angle of spectrometer

$N_{q}(I)=$ number observed per charge $q$ at fluxmeter setting $I$ From the concurrent work of Renken (1963).

$$
\begin{aligned}
& R_{c}=307 \\
& \Omega=1.47 \times 10^{-3} \text { steradian } \\
& q=9.28 \mu \text { coulomb }
\end{aligned}
$$

Then for $\theta_{1}=15^{\circ}$, and $\mathrm{d} \sigma / \mathrm{d} \Omega$ in mbarns/steradian, 


$$
n t=3.43 \times 10^{18} \times \frac{1}{\frac{d \sigma}{d n}} \times \int \frac{N_{g}(I)}{I} d I
$$

For the various elastic-scattering cross sections we used

$$
\begin{aligned}
& \mathrm{C}^{12}: 188 \mathrm{mb} / \text { ster } \quad \text { (Jackson 1953) } \\
& \mathrm{N}^{14}: 109 \mathrm{mb} / \mathrm{ster} \quad \text { (Tautfest 1955) } \\
& \text { O } 16 \text { : } 154 \mathrm{mb} / \text { ater } \quad \text { (Eppling 1953) } \\
& \mathrm{v}^{51}: 787 \mathrm{mb} / \mathrm{ster} \quad \text { (Rutherford 1911) }
\end{aligned}
$$

The scattering yield from a typical target is shown in Figure 4. and the calculated compositions for the various targets used are given in Table II. 


\section{APFENDIX B}

\section{Camera and Film Developing}

The optical properties of the Nikkor $\mathrm{f} / 1.1$ lens were measured on an optical bench. The focal point $F$ in object space was found to be 0.172 inch in front of the frontmo ot metal ring, the focal point $F^{\prime}$ in image space is 0.825 inch behind the rearmost metal piece, and the focal length was measured as 2.03 inches. The locations of the foci $F$ and $F^{\prime}$ and the corresponding principal planes $H$ and $H^{\prime}$ are shown in Figure 11, referred to the rear surface of the mounting ring. This stainless steel ring, 0.349 inch thick, has six 4-40 clearance holes on a 2.200-inch circle, and could be used to mount the lens to other equipment. The dimensions given correspond to a range setting on the lens of six feet; there is travel 0.064 inch either way from this position.

For a magnification of $1 / 3$, the film plane had to be $\frac{1}{3} \times f=0.68$ inch behind the plane $F^{\prime}$, as shown in Figure 11. The range over which the camera can be focused in this configuration is approximately one inch. If the space $r$ ring in Figure 11 is removed, the distance settings on the lens should approximately correspond to actual focus.

The film magazine has an electric motor and a microswitch which is operated by a cam turned by the moving film, If power is supplied to the motor long enough to move the cam far enough to throw the switch, the switch will then keep the motor on until the film has advanced one frame. A circuit using a silicon controlled rectifier (SCR) and a relay to start the film advance when triggered by a short pulse is shown in Figure 12. A buzzer and two neon lights are wired in 
parallel with the film-advance motor to warn the operator. Each advance is also counted on a mechanical register in the power supply unit.

The film used was Kodak Linagraph Pan, LP417 in 100-foot rolls. It was developed in a Morso G-3 rowind tank, using D-8 developer, as follows:

1. Prebath, Kodak "Photoflo," 2 min.

2. Develop, D-8, $68^{\circ} \mathrm{F}, 9$ min.

3. Stop, $1 \%$ HAc, 2 min.

4. Rinse, running water, 2 min.

5. Fix, fr "Repid Fixol" + liquid hardener, 6 min.

6. Rinse, running watex, 2 min.

7. Cleax, Kodak "Hypo Clearing Agent," 2 min.

8. Wash, running water, 6 min.

9. Photoflo, 2 min.

10. Dry.

Because the lens was used so close to the object, a serious barrel distortion was introduced, as shown in Figure 5a. Therefore an enlarger was constructed using the camera base plate and lens, and modifying a Beattie-Coleman ground glass holder for use as a film guide. Note that it is disastrous to use a bulb with too high wattage, since the cement used in the lens is subject to melting. In this case a 50-watt bulb was used, but it was run at about $60 \mathrm{~V}$ instead of $110 \mathrm{~V}$. Figure $5 \mathrm{~b}$ is a print made using this arrangement. 
APPENDEX C

Momentum of Gamma Rays

1. First excited state

The first excited state of $\mathrm{C}^{12}$ decays by emission of a 4.433$\mathrm{MeV} \gamma$-ray, adding a vector momentum $\overrightarrow{\mathrm{P}}_{\mathbf{y}}$ with magnitude 4.433 $\mathrm{MeV} / \mathrm{c}$ to the recolling nucleus. (See Figure 23.) Let $\mathrm{p}_{z}$ be a single component of the $\gamma$-ray momentum vector $\vec{p}_{\gamma}$, and let $W\left(p_{z}\right) d p_{z}$ be the probability that the value of the component lies between $P_{z}$ and $p_{z}+d p_{z}$. Assuming $\vec{p}_{Y}$ isotroplc, $W\left(p_{z}\right) d p_{z}$ is just the fraction of the surface of the sphere in momentum space of radius $\mathrm{P}_{Y}$ between

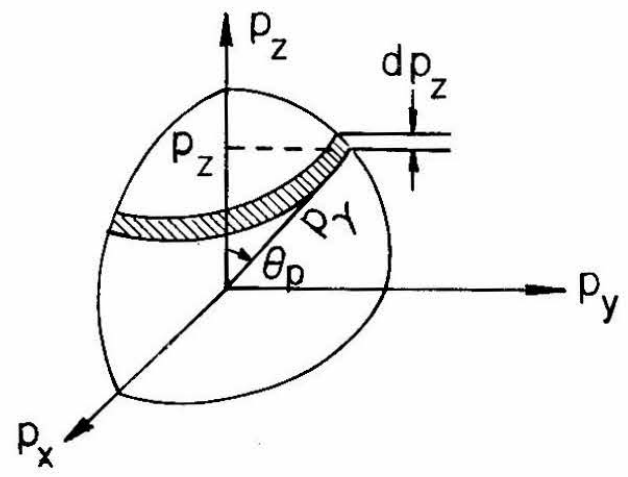

the planes $p_{z}$ and $p_{z}+d p_{z}$.

$$
\begin{aligned}
& W\left(p_{z}\right) d p_{z}=\frac{1}{4 \pi p_{Y}^{2}} \times 2 \pi\left(p_{\gamma} \sin \theta_{p}\right) \times \frac{d p_{z}}{\sin \theta_{p}} \\
& W\left(p_{z}\right) d p_{z}=\left\{\begin{array}{lc}
\frac{1}{2 p_{y}} d p_{z}, & p_{z}^{2}<p_{Y}^{2} \\
0, & p_{z}{ }^{2}>p_{Y}^{2} .
\end{array}\right.
\end{aligned}
$$

Since the angle of deflection of the recoll nucleus is small, its projection in the horizontal plane is just $\psi=p_{z} / p_{1}$, where $p_{1}$ is the Initial magnitude 
of the momentum of the recoil nucleus. Then

$$
\begin{aligned}
& p_{z}=p_{1} \psi \\
& W_{1}(\psi) d \psi=W\left[p_{z}(\psi)\right] d p_{z}(\psi)=\frac{1}{2 p_{\gamma}} \times p_{1} d \psi \\
& W_{1}(\psi) d \psi= \begin{cases}\frac{p_{1}}{2 p_{\gamma} d \psi,} & |\psi|<p_{\gamma} / p_{1} \\
0 & |\psi|>p_{\gamma} / p_{1} .\end{cases}
\end{aligned}
$$

Note that $W_{1}(\psi)$ given by equation $C 3$ is a function of $p_{1}$ and hence of the angle 6 . $W_{1}(\psi)$ for $\delta=0$ is plotted in Figure 24.

\section{Second excited state}

The $(0+)$ second etate of $C^{12}$ decays by E2-E2 $\gamma$-cascade through the $(2+)$ first state to the $(0+)$ ground state. If (9) is the anglo between the two $y$-rays, the angular correlation is

$$
W(\mu) d \mu=5 / 8\left(1-3 \mu^{2}+4 \mu^{4}\right) d \mu \text {, where } \mu=\cos \theta \text {. }
$$

In this case the magnitude $P_{Y}$ of the $Y$-momentum is not a constant, but will have a probability distribution $W\left(p_{Y}\right) d_{p_{\gamma^{\prime}}}$ and equation C3 will have to be integrated over all possible values of $p_{Y}$ :

$$
w_{2}(\psi) d \psi=\frac{p_{2}}{2} d \psi \int_{p_{2}|\psi|}^{p_{\max }} \frac{w\left(p_{y}\right)}{p_{y}} d_{p_{\gamma}} .
$$

where $p_{2}$ is the initial recoil-ion momentum, and the lower limit of the integration resulte from the limit in equation C3.

The magnitude $p_{Y}$ is given by the law of cosines: 


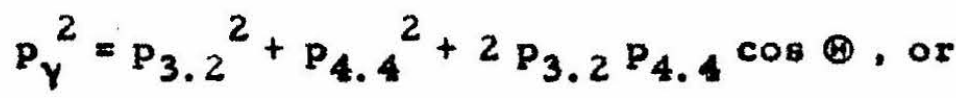

$$
\begin{aligned}
& p_{y}^{2}=a^{2}+b^{2} \mu
\end{aligned}
$$

where $a^{2}=30.039 \mathrm{MeV}^{2} / \mathrm{c}^{2}$ and $\mathrm{b}^{2}=28.575 \mathrm{MeV}^{2} / \mathrm{c}^{2}$.

Thus

$$
\begin{aligned}
& \mu=\frac{1}{b^{2}}\left(p_{Y}^{2}-a^{2}\right) \\
& d \mu=\frac{1}{b^{2}}\left(2 p_{y}\right) d p_{Y} .
\end{aligned}
$$

Having expressed $\mu$ as a function of $p_{\gamma}$, we can state

$$
\begin{aligned}
& W\left(p_{\gamma}\right) d p_{\gamma}=W\left[\mu\left(p_{\gamma}\right)\right] d \mu\left(p_{\gamma}\right)=W(\mu) \frac{d \mu}{d p_{\gamma}} d p_{\gamma} \\
& W\left(p_{\gamma}\right) d p_{\gamma}=5 / 8\left[1-3 \frac{1}{b^{4}}\left(p_{\gamma}^{2}-a^{2}\right)^{2}+4 \frac{1}{b^{8}}\left(p_{\gamma}^{2}-e^{2}\right)^{4}\right] \frac{2 p_{Y}}{b^{2}} d p_{\gamma}
\end{aligned}
$$

There are a maximum and a minimum value for $\mathrm{p}_{\mathbf{y}}$ :

$$
\begin{aligned}
& p_{\max }=p_{3.2}+p_{4.4}=\left(a^{2}+b^{2}\right)^{\frac{1}{2}}=7.656 \mathrm{MeV} / \mathrm{c} \\
& p_{\min }=\left|p_{3.2}-p_{4.4}\right|=\left(a^{2}-b^{2}\right)^{\frac{1}{2}}=1.210 \mathrm{MeV} / \mathrm{c}:
\end{aligned}
$$

the value of $W\left(p_{\gamma}\right) d_{Y}$ is zero outside these limits. Note that

$$
\int_{p_{\min }}^{P_{\max }} w\left(p_{Y}\right) d p_{Y}=1 .
$$

Letting $F\left(p_{\gamma}\right)=\int \frac{W\left(p_{y}\right)}{{ }^{p_{y}}} d p_{y}$, we have from equation C5 
$W_{2}(\psi) d \psi=\left\{\begin{array}{l}0, \quad|\psi|>p_{\max } / p_{2} \\ \frac{p_{2}}{2}\left[F\left(p_{\max }\right)-F\left(p_{2}|\psi|\right)\right] d \psi, \frac{p_{\min }}{p_{2}} \leqslant|\psi|<\frac{p_{\max }}{p_{2}} \\ W_{2}\left(\frac{p_{\min }}{p_{2}}\right) d \psi, \quad|\psi|<p_{\min } / p_{2}\end{array}\right.$

and

$$
\begin{gathered}
F\left(p_{Y}\right)=\frac{5}{4 b^{2}}\left[\left(1-3 \frac{a^{4}}{b^{4}}+4 \frac{a^{8}}{b^{8}}\right) p_{Y}+\frac{1}{3}\left(6 \frac{a^{2}}{b^{4}}-16 \frac{a^{6}}{b^{8}}\right) p_{Y}^{3}\right. \\
\left.+\frac{1}{5}\left(-\frac{3}{b^{4}}+24 \frac{a^{4}}{b^{8}}\right) p_{Y}^{5}-\frac{16}{7} \frac{a^{2}}{b^{8}} p_{Y}^{7}+\frac{4}{9} \frac{1}{b^{8}} p_{Y}^{9}\right] .
\end{gathered}
$$

Like the equation C3, the distribution CII is also a function of the angle $\delta$ (see Figure 23) through the recoil-ion momentum $p_{2}$. It is plotted in Figure 24 for $8=0$. The calculation was done on the IBM 7090 as a part of the calculation of $G$. 


\section{APPENDIX D}

\section{Projection of a Rectangle in Spherical Co-ordinates}

A rectangular slit, such as used to define the solid angle of the alpha counter in this experiment, does not present a rectangular opening in $(\phi, \theta)$ space; the farther the slit ia from the "equator" $(\theta=\pi / 2)$, the more curved the projection will be, with the extremities pointed toward the equator. Also, if the slit is not wort compared to its dis tance from the center of the sphere, the ends will subtend a smaller angle than the central portion.

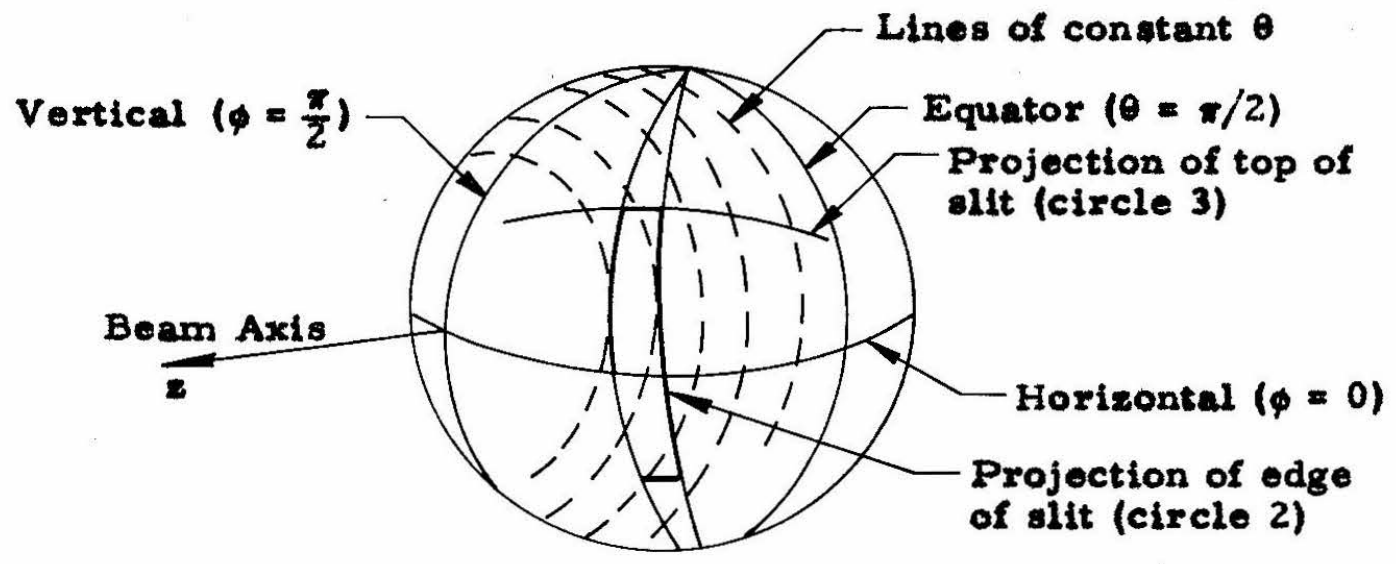

We shall make use of the properties of a right spherical triangle. If $a, b$, and $c$ are three intersecting great-circle arcs, and $A$, $B$, and $C$ are the angles opposite the sides $a, b$, and $c$, and if angle $c=\pi / 2$, then

$$
\begin{aligned}
\sin a & =\sin A \sin C \\
& =\tan b \cot B
\end{aligned}
$$




$$
\begin{aligned}
\cos A & =\cos a \sin B \\
& =\tan b \cot C \\
\cos C & =\cot A \cot B \\
& =\cos a \cos b
\end{aligned}
$$

Take the slit to be of width $w$, height $h$, centered at $\theta=\theta_{0}$ and $\phi=0$, and at a distance $r$ from the center of the sphere. We consider only the quadrant $0<\theta \leqslant \pi / 2$; the pole cannot be included without added complications. The vertical line forming either edge of the slit will project onto the sphere as a great circle perpendicular to the horizontal $(\phi=0)$ circle. Let $\theta_{1}=\theta_{0}-\operatorname{atan}(w / 2 r)$ be the value of $\theta$ at which the edge nearest the pole intersects $\phi=0$, and call its projection circle 1. Every latitude circle with $\theta>\theta_{1}$ will intersect circle 1; let the value of $\phi$ at the intersection be $\phi_{1}(\theta)$. Then arcs of $\phi=0$, $\dot{\phi}=\phi_{1}(\theta)$, and circle 1 form a right spherical triangle. The length of the $\dot{\phi}=0$ arc is $\theta_{1}$, and the length of the $\phi=\phi_{1}(\theta)$ arc is $\theta$; the angle

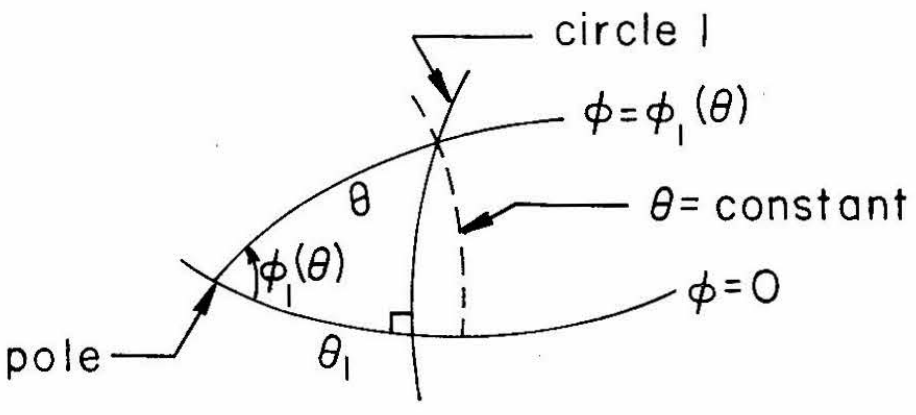

between these two arcs is $\phi_{1}(\theta)$. From equation D4,

$$
\cos \phi_{1}(\theta)=\tan \theta_{1} \cot \theta
$$

Similarly for the other edge of the slit, let $\theta_{2}=\theta_{0}+\operatorname{atan}(w / 2 x)$ be the 
intercept with $\phi=0$. Then for $\theta \geqslant \theta_{2}$, the intersection of the projection (circle 2) with latitude $\theta$ will be at $\phi_{2}(\theta)$ :

$$
\cos \dot{\phi}_{2}(\theta)=\tan \theta_{2} \cot \theta
$$

The top (or bottom) edge of the slit projects onto the sphere as a great circle inclined at an angle $\phi_{0}=\operatorname{atan}(\mathrm{h} / 2 \mathrm{r})$ to the horizontal. Call this projection circle 3; it will intersect the horizontal at $\theta=\theta_{0}$ 尛/2. hence at a distance of $\left(\pi / 2-\theta_{0}\right)$ from the pole. To find the distance $\theta_{3}$ of circle 3 from the pole, conatruct the perpendicular to circle 3 throurh

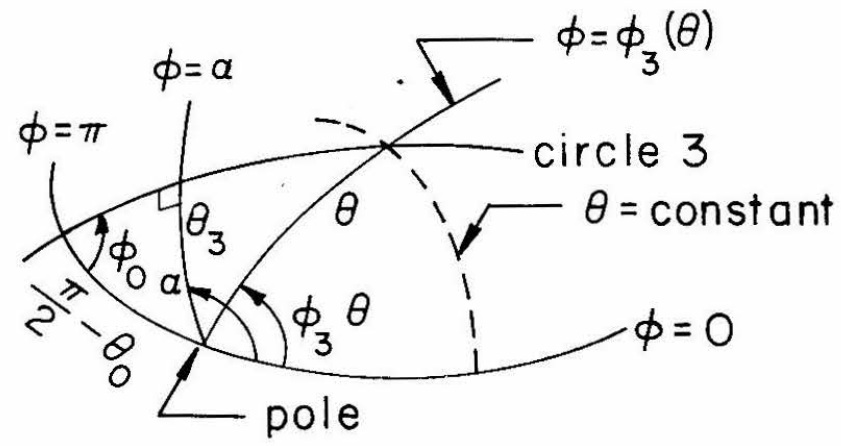

the pole. This will be a circle of constant $\phi$ s let $\phi=a$ be itz equation. From the right spherical triangle thus formed with $\phi=\pi$ and circle 3 , asing equation $\mathrm{Dl}$.

$$
\sin \theta_{3}=\sin \phi_{0} \sin \left(\pi / 2-\theta_{0}\right)=\sin \phi_{0} \cos \theta_{0} .
$$

and vaing equation D4,

$$
\begin{aligned}
& \cos (\pi-\alpha)=\tan \theta_{3} \cot \left(\pi / 2-\theta_{0}\right) \\
& \cos a=-\tan \theta_{3} \tan \theta_{0} .
\end{aligned}
$$

Note that for $0<\theta \leqslant \pi / 2$, we have $0<\theta_{3}<\phi_{0}$ and $\alpha>\pi / 2$. 
Any latitude circle $\theta>\theta_{3}$ will intersect circle 3 in two places. However one of these will be at an angle greater than $\alpha$ anc hence greater than $\pi / 2$; it has no significance unless the slit is wide onough to include the pole. Let $\phi_{3}(\theta)$ be the co-ordinate of the intersection 1 ess than $\alpha$. Then from the right spherical triangle formed by $\phi=\phi_{3}(\theta)$, $\phi=\alpha$, and circle 3 , with reepective arc length $\theta, \theta_{3}$, and unknown, using equation D4.

$$
\cos \left[\alpha-\phi_{3}(\theta)\right]=\tan \theta_{3} \cot \theta
$$

To summarize, the calculation proceded as follows. Given $\theta_{0}$, $h, w$, and $r$, we have

$$
\begin{aligned}
& \theta_{1}=\theta_{0}-\operatorname{atan}(w / 2 r), \\
& \theta_{2}=\theta_{0}+\operatorname{atan}(w / 2 r), \\
& \phi_{0}=\operatorname{atan}(h / 2 r), \\
& \theta_{3}=\operatorname{asin}\left(\sin \phi_{0} \cos \theta_{0}\right) . \\
& \alpha=\operatorname{acos}\left(-\tan \theta_{3} \tan \theta_{0}\right) .
\end{aligned}
$$

These parameters describe the size of the slit and its position. As functions of polar angle $\theta$, the equations $\phi(\theta)$ for the sides and top of the slit are:

$$
\phi_{1}(\theta)= \begin{cases}\operatorname{acos}\left(\tan \theta_{1} \cot \theta\right), & \theta>\theta_{1} \\ 0, & \theta \leqslant \theta_{1}\end{cases}
$$




$$
\begin{aligned}
& \dot{\phi}_{2}(\theta)= \begin{cases}\operatorname{acos}\left(\tan \theta_{2} \cot \theta\right), & \theta>\theta_{2} \\
0, & \theta \leqslant \theta_{2}\end{cases} \\
& \dot{\phi}_{3}(\theta)= \begin{cases}a-\operatorname{acos}\left(\tan \theta_{3} \cot \theta\right), & \theta \geqslant \theta_{3} \\
x_{1} & \theta<\theta_{3} .\end{cases}
\end{aligned}
$$

The values of $\phi$ outside the limits of $\theta$ within which they were derived are defined as above for convenience.

The maximum value of $\phi$ which is within the slit opening at a given $\theta$ is either $\dot{\varphi}_{1}(\theta)$ or $\phi_{3}(\theta)$, whichever is smaller; the lower limit (non-negative) $\phi$ is $\dot{\phi}_{2}(\theta)$. Thus the $\Delta \phi$ opening of the slit as a function of $\theta$ is $R(\theta)$, given by

$$
\frac{1}{2} R(\theta)=\min \left[\phi_{1}(\theta), \phi_{3}(\theta)\right]-\dot{\phi}_{2}(\theta)
$$

The solid angle of the slit is

$$
\mathrm{d} \Omega=\int_{0}^{\pi / 2} R(\theta) \sin \theta \mathrm{d} \theta
$$

The shape of $a$ slit $u$ sed in the experiment $h=0.200$ inch, $w=0.060$ inch, $x=0.625$ inch) is shown in Figure 25 . It was used at $\theta_{0}=67.18^{\circ}$; it was also calculated at $\theta_{0}=10^{\circ}$ for contrast. The solid angle d $\Omega$ was 0.0303 steradians, independent of position. 


\section{REEERENCES}

F. Ajzenberg-Selove and T. Lauritsen, 1959, Nuclear Physics 11, 1. (See Figure 1.)

F. Ajzenberg-Selove and P. F. Stelson, 1960, Phys. Rev. 120, 500. (See text pp. 3,4.)

D. E. Alburger, 1960, Fhys. Rev. 118, 235. (See text pp. 3,4.)

D. E. Alburger, 1961, Phys. Rev. 124, 193. (See text pp. 4, 37.)

M. Bader, R. E. Pixley, F. S. Mozer, and W. Whaling, 1956, Phys. Rev. 103. 32. (See text p. 34.)

A. B. Brown, C. W. Snyder, W. A. Fowler, and C. C. Lauritsen, 1951, Phys. Rev. 82, 159. (See text p. 16.)

$B^{2}$ FH: E. M. Burbidge, G. R. Burbidge, W. A. Fowler, and F. Foyle, 1957, Revs. Modern Phys. 29, 547. (See text pp. 3, 37.)

C. W. Cook, W. A. Fowlex, C. C. Lauritsen, and T. Lauritsen, 1957, Phys. Rov. 107, 508. (See text pp. 1-3.)

G. Dearnaley and A. B. Whitehoad, 1961, Nucl. Instr. and Meth. 12, 205. (See text p. 10.)

D. N. F. Dunbar, R. E. Pixley, 涌.A. Wenzel, and W. Whaling, 1953, Phya. Rev. 92, 649. (See text pp. 1, 5, 28.)

S. F. Eccles and D. Bodansky, 1959, Phys. Rev. 113,608. (See text p. 3.)

F. J. Eppling, J. R. Cameron, R. H. Devis, A. S. Divatia, A. I. Calonsky, E. Goldberg, and R. W. Hill, 1953, Phys. Rev. 91, 438A; also F. J. Eppling. Thesis, 1953, University of Wisconsin. (See Appendix A, p。 43.) 
Everling, König, Mattauch, and Wapatra, 1960, Nuclear Fhysics 15 , 342. (See text p. 2.)

W. A. Fowler and F. Hoyle, 1962, "Neutrino Processes in Supervovae," to bo published. Also W. A. Fowler, private communication. (See text, p. 38.)

H. Frauenfelder, 1955, Beta- and Gamma-Ray Spectroscopy, K. Siegbahn, od., p. 556 (North-Holland Publishing Co., Amsterdam). (See text p. 32, Appendix C, p. 47.)

J. F. Fregeau, 1956, Phys. Rev. 104, 225. (See text p. 4.)

Gmelins Handbuch der Anorganischen Chemie, 1936, 8 Auflage, Nummer 36, 8.71. (Verlag Chemie, G. zn. b. H., Berlin, 1936.) (See text p. 7.)

D. E. Groce, J. H. McNally, and $\forall$. Whaling, 1962, Bull. Am. Phys. Soc. 1, 453. (See text p. 28.)

A. Hemmendinger, 1948, Phys. Rev. 73, 806; Phys, Rev. 75, 1267 (1949). (See text p. 1.)

F. Hoyle, D. N. F. Dunbax, Y. A. Fenzel, and W. Whaling, 1953, Fhys. Rev. 92, 1095. (See text pp. 1, 5, 28.)

E. Hoyle, 1954, Astrophyz. J. Suppl. 1, 121. (See text p. 1.)

H. L. Jackson, A. 1. Galonsky, F. J. Eppling, R. W. Hill, E. Coldberg. anci J. R. Cameron, 1953, Fhys, Rev. 89, 365. (See Appendix A. p. 43.)

R. W. Kavanagh, 1958, Bull. Am. Fhys. Soc. 3, 316. (See text p. 3.) C. C. Lauritsen and T. Lauritsen, 1948, Rev. Sci. Instr. 19, 916. (See text p. 16.) 
T. Lauritsen and $F$. Ajrenberg-Selove, 1962, "Energy Levels of Light Nuclei," Nucleer Data Sheeta. 1961, Sets 5 and 6. (See Figure 1.) H. J. Martin and A. A. Kraus, 1957, Rev. Sci. Instr. 28, 175; also H. J. Martin, thesis, Callfornia Institute of Technology, 1956. (See toxt p. 7.)

E. J. Öpik, 1951, Proc. Roy. Irish Acad. A54, 49. (See text p. 1.) E. J. Öplk, 1953, Mem. Soc. Roy. Sci. Liðge 14, 131. (See text p. 1.) J. R. Oppenheimer and J. S. Schwinger, 1939, Phys. Rev. 56, 1066. (See text p. 4.)

J. H. Renken, 1963, Thesis, California Institute of Technology (unpub1iahed). (See Appendix A, p. 42.)

H. K. Reynolda, D. N. F. Dunbar, W. A. Wenzel, and W. Whaling, 1953, Phys. Rev. 92, 742. (See text p. 34.)

E. Rutherford, 1911, Phil. Mag. 21, 669. (See Appendix A.)

E. E. Salpeter, 1952, Ap. J. 115, 326; Annual Reviews of Nuclear Science (Annual Reviews, Inc.. Stanford, 1953), Vol. 2, p. 41. (See text p. 1.)

E. E. Salpeter, 1954, Australian J. Fhys. 7. 373. (See text p. 3.) E. E.Salpeter, 1957, Phys. Rev. 107, 516. (See text pp. 1,2,37.) L. I. Schiff, 1955, Phys. Rev. 98, 1281. (See text p. 4.) R. J. Sime and J. L. Margrave, 1956, J. Phys. Chem. 60, 810. (See text p. 7.)

C. W. Snyder, S. Rubin, W. A. Fowler, and C. C. Lauritsen, 1950, Rev. Scl. Instr. 21, 852. (Seo Appendix A.) 
$-58-$

G. W. Tautfest, J. R. Havill, and S. Rubin, 1955, Phy3. Rev. 98, 280A. (See Appendix A, p. 43.)

A.V. Tollestrup, W. A. Fowler, and C. C. Lauritsen, 1949, Dhys. Rev. 76, 428. (See text p. 1.) 


\section{Tablo I}

Kinematics of $\mathrm{N}^{14}(\mathrm{~d}, a) \mathrm{C}^{12}$

For a deuteron energy of $1.800 \mathrm{MeV}$, the relativistically cor rect energies of $\mathrm{c}^{12}$ tons at $90^{\circ}$ in the $\mathrm{lab}$, the corresponding $\alpha$ energies, the lab angle $\theta$ of the $\alpha^{\prime} s$, and the flight time $t$ of the $c^{12}$ ions through $85 \mathrm{~cm}$ are given. The ground state of the reaction $\mathrm{C}^{13}(\mathrm{~d}, \mathrm{a}) \mathrm{B}^{11}$ is also included. (See text pp. 6, 23.)

\begin{tabular}{|c|c|c|c|c|}
\hline State & Ion energy & $a$ energy & $\theta$ & $\mathbf{t}$ \\
\hline$c^{12}$ ground & $3.620 \mathrm{MeV}$ & 11. $759 \mathrm{MeV}$ & $73.9^{\circ}$ & $112 \mathrm{nsec}$ \\
\hline $\mathrm{C}^{12 *}(4.433 \mathrm{MeV})$ & 2.511 & 8.435 & 70.9 & 134 \\
\hline $\mathrm{C}^{12 *}(7.656 \mathrm{MeV})$ & 1.706 & 6.017 & 67.2 & 162 \\
\hline $\mathrm{B}^{11}$ ground & 1.616 & 5.351 & 65.8 & 160 \\
\hline
\end{tabular}


$\mathrm{Table} \mathrm{II}$

Target Composition

Compositions of various targets in units of $10^{17}$ atoma $/ \mathrm{cm}^{2}$ calculated as in Appendix A from yield curves eimilar to Figuro 4. (See text p. 7.)

\begin{tabular}{l|llll} 
Taxget & $\mathrm{C}^{12}$ & $N^{14}$ & $0^{16}$ & $V^{51}$ \\
\hline C4-XI & 22.4 & 2.6 & 0.2 & 3.1 \\
C5-I & 15.6 & 0.3 & 0.1 & 0.4 \\
C5-II & 14.5 & 0.7 & 0.3 & 0.8 \\
C6-I & 16.6 & 1.4 & 0.1 & 1.3 \\
C6-VII & 19.1 & 3.3 & 0.5 & 4.0 \\
C6-IX & 19.6 & 2.8 & 0.5 & 4.3 \\
C6-XII & 22.1 & 3.1 & 0.4 & 4.9 \\
C7-XVI & 22.4 & 1.2 & 0.3 & 1.3
\end{tabular}


Table III

Input Data and Calculated Normalization

The fixst column gives the identification (film and frame) number of each event; the data presented here are frames 320 through 416 of film \#15, and frame 336 is a double exposure. The next five columns are the input data: 100 channel address, 400 channel address, upper-beam pulse height in $\mathrm{cm}$, lower-beam pulse height in $\mathrm{cm}$, and horizontal separation (positive if lowex-beam pulse is to the right) in cm. The final five columns are the energy and time equivalents of the five data columns. E100 and E UP refer to the alpha-particle energy in $\mathrm{MeV}$, and $\mathrm{E} 400$ and $\mathrm{E}$ LOW to the $\mathrm{C}^{12}$ recoil-ion energy in MeV. DT is the time separation (difference from $162 \mathrm{nsec}$ ) in nsec. Five frames included in this table represent $C^{12}(3+)-\alpha_{2}$ events; they are 338,356 . 362, 373, and 405. (See text pp. 20-22.) 
$-61-$

TABLE III

\begin{tabular}{|c|c|c|c|c|c|c|c|c|c|c|}
\hline \multirow[b]{2}{*}{ NUMBER } & \multicolumn{5}{|c|}{ DATA } & \multicolumn{5}{|c|}{ CALCULATED } \\
\hline & Cr-1 & $\mathrm{CH}_{4}$ & CNUP & CNLC & CNCT & E $1 \mathrm{CO}$ & $E 400$ & E UP & E LOW. & DI \\
\hline $1532 \mathrm{C}$ & $-c$. & 63 . & $0 .{ }^{\circ} \mathrm{C}$ & $1.7 \mathrm{C}$ & C.9 & -3.55 & 2.45 & 3.55 & 2.47 & 14. \\
\hline 15321 & 24. & 71. & $1.0^{\circ} \mathrm{C}$ & C.SC & C. 5 & 7.91 & 2.76 & 8.43 & 1.04 & 6. \\
\hline 15327 & 7. & 38. & $1 . ? 1$ & 1.21 & C. 5 & 5.70 & 1.48 & 5.37 & 1.60 & 6. \\
\hline 15328 & $-c$. & -0 . & 1.91 & $1 . C \mathrm{C}$ & $-c .7$ & -8.48 & -1.22 & 8.48 & 1.22 & -18 \\
\hline 15329 & 52. & 30 . & 2.52 & C.87 & C.9 & 11.55 & 1.17 & 11.18 & 0.99 & 14. \\
\hline $1533 \mathrm{C}$ & $-c$. & -0 . & 2.58 & 2.30 & -1.7 & -11.45 & -3.54 & 11.45 & 3.54 & -38. \\
\hline 15331 & $-c$. & -0 . & 2.72 & 2.31 & $-c$ & -9.85 & -3.56 & 9.85 & 3.56 & -4 \\
\hline 15334 & 5. & 36. & $1 .>0$ & 1.26 & C. 5 & 5.44 & 1.40 & 5.33 & 1.69 & 6. \\
\hline 15336 & $-c$. & -0 . & 1.20 & C.SC & $-C .2$ & -5.77 & $-1 \cdot 0_{4}$ & 5.77 & 1.04 & -8 \\
\hline-15336 & 7. & 36. & 1.26 & 1.10 & $-c$ & 5.70 & 1.40 & 6.04 & 1.40 & -4. \\
\hline 15338 & 7. & 44. & $1.2 t$ & 1.35 & C. 2 & 5.70 & 1.71 & 6.04 & 1.85 & 0 \\
\hline 15341 & 5. & 33. & 1.16 & 1.07 & $-c .2$ & 5.44 & 1.28 & 5.15 & 1.35 & -8 \\
\hline 15342 & $\varepsilon$. & 43. & 1.73 & 1.20 & C.I & 5.83 & 1.67 & 5.46 & 1.58 & -2 \\
\hline 15343 & 51. & 32. & $2 .+0$ & C. $9 \mathrm{C}$ & $-c .3$ & 11.42 & 1.24 & 11.54 & 1.04 & -10 \\
\hline 15345 & $-c$. & 35. & $2 .=4$ & C. 87 & C. 4 & -11.27 & 1.36 & 11.27 & 0.99 & 4. \\
\hline $1534 t$ & 22. & 43. & 1.91 & $1.4 \mathrm{C}$ & c. 2 & 7.65 & 1.67 & 8.48 & 1.94 & 0. \\
\hline 15347 & 23. & 46. & $1 .{ }^{\circ} \mathrm{C}$ & 1.47 & C. 2 & 7.78 & 1.79 & 8.43 & 2.06 & U. \\
\hline 15348 & $1 c$. & 32. & 1.49 & C. 54 & $-c .2$ & 6.09 & 1.24 & 6.61 & 1.12 & -8 \\
\hline 15349 & $3 C$. & 33. & 1.98 & $1 . \mathrm{CC}$ & $-c .6$ & 8.69 & 1.28 & 8.79 & 1.22 & -16 \\
\hline 15350 & 53. & 33. & 2.53 & C. $8 \mathrm{C}$ & $-C .1$ & 11.68 & 1.28 & 11.23 & 0.87 & -6 \\
\hline 15352 & 25. & 31. & $1.9 \mathrm{C}$ & 1.CC & C. 2 & 8.56 & 1. 20 & 8.43 & 1.22 & 0. \\
\hline 15353 & $2 \varepsilon$. & 42. & $1 . c^{1}$ & 1.25 & $c .3$ & 8.43 & 1.63 & 8.48 & 1.67 & 2 \\
\hline 15354 & IC. & 29. & 1.78 & $1 . C C$ & $1 . \mathrm{C}$ & 6.09 & 1.13 & 6.12 & 1.22 & 16. \\
\hline $1535 t$ & $1 C$. & 48 . & 1.42 & 1.62 & C. 1 & $t . C y$ & 1.87 & 6.30 & 2.33 & -2 \\
\hline 15357 & $-c$. & 31. & 1.71 & 1.04 & C. 3 & -5.37 & 1.20 & 5.37 & 1.29 & 2 \\
\hline 15359 & $3 c$. & 31. & 1.91 & $C .93$ & C. 4 & 8.69 & 1.20 & 8.48 & 1.10 & 4. \\
\hline 15361 & 31. & 31. & 1.03 & C.54 & $-c .7$ & $\varepsilon .82$ & 1.20 & 8.56 & 1.12 & $-1 \varepsilon$ \\
\hline 15362 & 12. & 47. & 1.42 & 1.37 & C.1 & 6.35 & 1.83 & 6.30 & 1.88 & -2 \\
\hline 15363 & 28 . & 28 . & $1.9 \mathrm{C}$ & $1 . C 8$ & 1.3 & 8.43 & 1.09 & 8.43 & 1.37 & 22 \\
\hline 15364 & 7. & 31. & 1.70 & 1.CC & -1.1 & 5.70 & 1.20 & 5.33 & 1.22 & -26 \\
\hline 15365 & 27 . & $\in 8$. & $1 .<1$ & 1.51 & -1.2 & 8.30 & 2.64 & 8.48 & 2.85 & -28. \\
\hline 15367 & $2 \varepsilon$. & 32. & 1.01 & $1.1 \mathrm{C}$ & 1.C & 8.43 & 1.24 & 8.48 & 1.40 & 16 \\
\hline 15368 & 53. & 28 . & $2 .+1$ & C. 87 & C. 7 & 11.68 & $1 . C 9$ & 11.58 & 0.99 & 10. \\
\hline 15369 & 7. & 39. & 1.77 & 1.11 & C.S & 5.70 & 1.52 & 5.64 & 1.42 & 6. \\
\hline $1537 \mathrm{C}$ & 51. & 32. & $2 .=0$ & $c .83$ & C. 9 & 11.42 & 1.24 & 11.09 & 0.92 & 14. \\
\hline 15373 & s. & 46. & 1.29 & 1.48 & C.I & 5.96 & 1.79 & 6.17 & 2.08 & -2 \\
\hline 15374 & 11. & 32. & $1.2 t$ & 1. CC & -1.3 & 6.22 & 1.24 & 6.04 & 1.22 & -30 \\
\hline 15375 & 20. & 54. & 1.73 & $1 . t t$ & $-C .1$ & 7.39 & 2.10 & 7.68 & 2.40 & -6 \\
\hline $1537 t$ & 25. & 32. & 1.08 & C.SC & $-1 . c$ & 8.56 & 1.24 & 8.34 & 1.04 & -24. \\
\hline 15377 & 51. & 33. & 2.56 & $1 . \mathrm{Cc}$ & $-1 \cdot 3$ & 11.42 & 1.28 & 11.36 & 1.22 & -30 \\
\hline 15378 & 12. & 30. & 1.40 & C.85 & C. 3 & 6.35 & 1.17 & 6.21 & 0.96 & 2. \\
\hline 15379 & s. & 33. & 1.21 & 1.12 & $-C .2$ & 5.56 & 1.28 & 5.81 & 1.44 & -8 \\
\hline 15381 & 27. & 46. & 1.03 & 1.53 & C. 2 & 8.30 & 1.79 & 8.56 & 2.17 & 0 \\
\hline 15384 & 27. & 31 . & 1.97 & $1 . C C$ & -1.1 & 8.30 & 1.20 & 8.74 & 1.22 & -26 \\
\hline 15385 & 33. & 31. & $2 . r c$ & $1 . C C$ & -1.1 & S.C8 & 1.20 & 8.88 & 1.22 & -26 \\
\hline 15388 & 49. & 32. & 2.58 & C.SC & $c .3$ & 11.16 & 1.24 & 11.45 & 1.04 & 2 \\
\hline 15389 & $3 C$. & $3 C$. & 1.95 & 1. CC & $-C .4$ & $\varepsilon, \in 9$ & 1.17 & 8.65 & 1.22 & -12 \\
\hline $1539 \mathrm{C}$ & 33. & 30. & 2.56 & C.SC & $-c .1$ & 5.08 & 1.17 & 9.14 & 1.04 & -6 \\
\hline 15392 & $t$. & 39. & $1.7 \mathrm{C}$ & 1.36 & C. 3 & 5.57 & 1.52 & 5.33 & 1.86 & 2. \\
\hline 15393 & $-c$. & 31. & 1.92 & 1.00 & -1.3 & -8.52 & 1.20 & 8.52 & 1.22 & -30 \\
\hline 15397 & $-c$. & $-c$. & 1.03 & C.SC & $-c .8$ & -8.56 & -1.04 & 8.56 & 1.04 & -20 \\
\hline 15400 & $5 C$. & 32. & $2.5 \mathrm{C}$ & C.85 & C. 6 & 11.29 & 1.24 & 11.09 & 0.96 & 8. \\
\hline 15401 & $-c$. & 33. & 1.92 & C.\&7 & $-c .6$ & -8.52 & 1.28 & 8.52 & 0.99 & -16. \\
\hline 15403 & $2 \varepsilon$. & 33. & $1 .{ }^{\circ} \mathrm{C}$ & 1.22 & C. 4 & 8.43 & 1.28 & 8.43 & 1.61 & 4. \\
\hline 15405 & 11. & 42. & 1.42 & 1.41 & C. 3 & 6.22 & 1.63 & 6.30 & 1.95 & 2. \\
\hline 15406 & 27. & 57. & 1.91 & $C . S C$ & C. 3 & $8.3 C$ & 2.21 & 8.48 & 1.04 & 2. \\
\hline 15407 & 11. & 66. & 1.44 & $1.5 \mathrm{C}$ & $-c .5$ & 6.22 & 2.56 & 6.39 & 2.83 & -14. \\
\hline $154 c 8$ & 29. & 29. & 1.91 & C.SC & $-c .2$ & 8.56 & 1.13 & 8.48 & 1.04 & -8. \\
\hline 15409 & 52. & 31. & $2 . \times C$ & $1 . C C$ & $-C .3$ & 11.55 & 1.20 & 11.54 & 1.22 & -16. \\
\hline $1541 \mathrm{C}$ & 9. & 43. & 1.28 & 1.49 & C. 3 & 5.56 & 1.67 & 6.12 & 2.10 & 2. \\
\hline 15416 & $2 s$. & $\in 8$. & 1.96 & $2 . C C$ & $-1 \cdot 3$ & 8.56 & 2.64 & 8.70 & 3.01 & $-3 c$. \\
\hline
\end{tabular}


Table IV

Summary of Experimental Data

Fifteen runs are listed with information pertinent to calculations. "Counts" is the number of $\mathrm{C}^{12}(3+)-\mathrm{Cl}_{2}$ events observed, $\alpha=\left(\psi_{1}+\alpha_{0}\right) / 10,000$ is a measure of the amount of bombardsarst, and $R_{1}=C^{12}(4 t)-q_{1}$ coincidence rate per $10,000\left(w_{1}+c_{0}\right)$ is a measure of the efficiency. (See text p. 24.)

\begin{tabular}{|c|c|c|c|c|c|c|c|}
\hline \multirow[b]{2}{*}{ Run } & \multirow[b]{2}{*}{ Target } & \multirow[b]{2}{*}{$\alpha$ sltt } & \multicolumn{2}{|c|}{ Fraxaes } & \multirow[b]{2}{*}{ Counts } & \multirow[b]{2}{*}{$c$} & \multirow[b]{2}{*}{$R_{1}$} \\
\hline & & & First & Last & & & \\
\hline 1 & C6-1X & $0.032^{\prime \prime}$ & 3731 & 5535 & 10 & 332 & $420 \div 40$ \\
\hline 2 & & & 5588 & 5893 & 2 & 94.8 & $370 \div 15$ \\
\hline 3 & C6-XII & & 7581 & 8897 & 10 & 165.0 & $420 \div 40$ \\
\hline 4 & C7-XVI & & 9641 & 10275 & 7 & 111.4 & $460 \pm 20$ \\
\hline 5 & & & 10321 & 11878 & 18 & 348.3 & $530: 25$ \\
\hline 6 & & & 12048 & 12529 & 6 & 94.9 & $510 \pm 20$ \\
\hline 7 & & 0.062 & 12542 & 13137 & 0 & 34.5 & $310 \pm 15$ \\
\hline 8 & & 0.060 & 13146 & 14897 & 12 & 162.3 & $340 \pm 15$ \\
\hline 9 & C6-I & & 15007 & 15892 & 26 & 422.1 & $460 \pm 20$ \\
\hline 10 & & & 16008 & 16896 & 14 & 225.9 & $450 \pm 30$ \\
\hline 11 & & & 17008 & 17895 & 12 & 279.0 & $365 \pm 25$ \\
\hline 12 & C6-V III & & 18009 & 18898 & 9 & 424.8 & $325 \pm 20$ \\
\hline 13 & & & 20007 & 20897 & 15 & 421.3 & $380 \div 15$ \\
\hline 14 & & & 21012 & 21896 & 17 & 501.5 & $370: 20$ \\
\hline 15 & & & 22008 & 22890 & 12 & 385.3 & $305 \pm 30$ \\
\hline $\begin{array}{l}\text { back- } \\
\text { ground }\end{array}$ & C7-XIV & & 23008 & 23284 & 0 & 12.2 & $113: 25$ \\
\hline
\end{tabular}




\section{Table V}

Counting Rates of Observed Charge States

Recoil $\mathrm{C}^{12}$ ions from the reaction $\mathrm{C}^{12}\left(\mathrm{He}^{4}, \mathrm{C}^{12}\right) \mathrm{He}$ were observed. The bombarding energies $E_{1}$ necessary to give the desired recoil-ion energies are shown, and the counting rate per monitor alpha particle observed with a separate counter in the target chamber is given for each observed charge state. Each entry in the table is an average of three or more runs. The quoted errors are generally the opread of the different readings, except in a few cases in which the statistical error in the number of counts was greater. Entries not observed due to limitations of the equipment are indicated by asterisks (*), and were assumed negligible. (See text p. 29.)

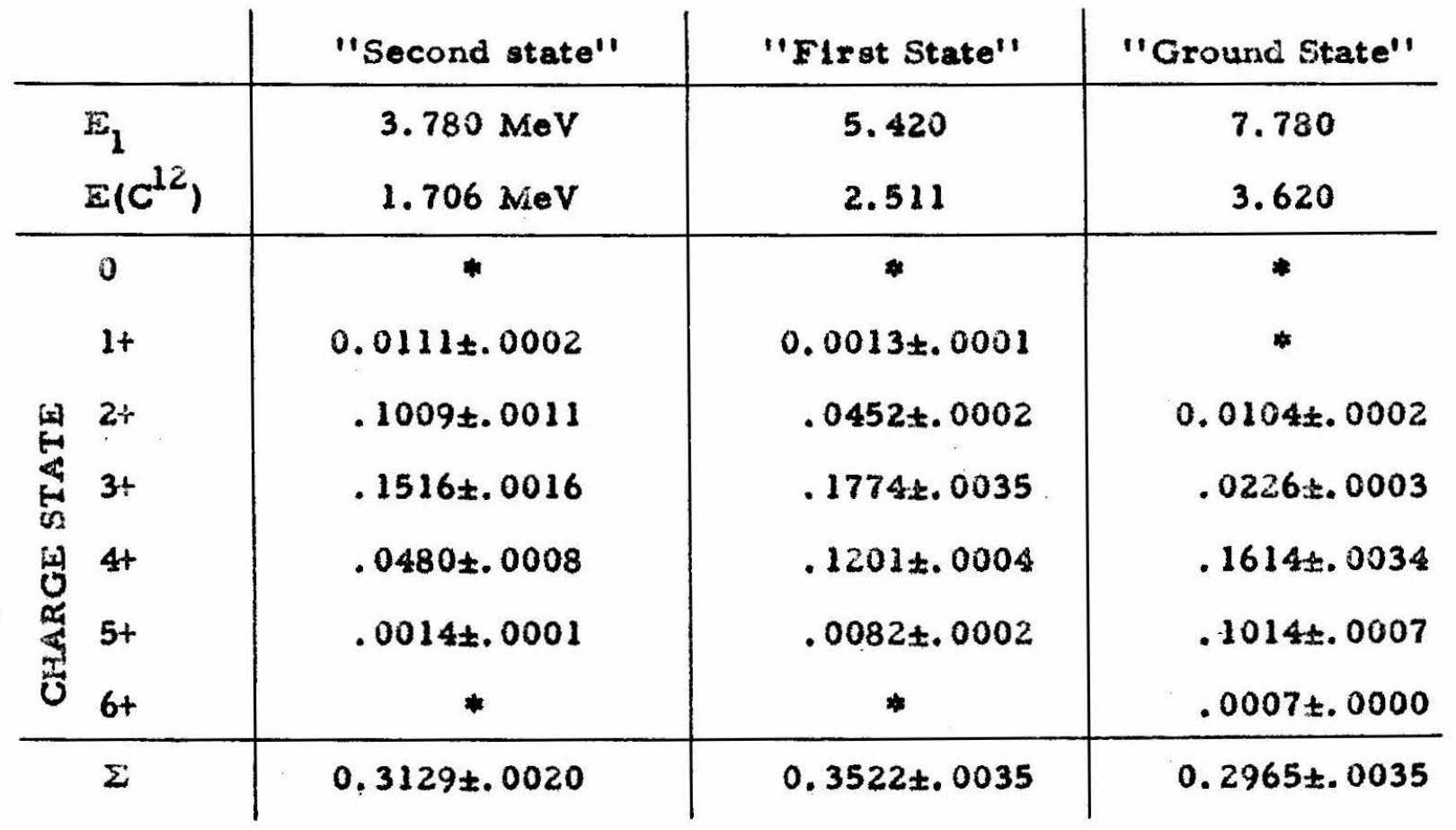


$-64-$

Table VI

Ratios of Ionic-Charge States

Charge-state ratios for $\mathrm{C}^{12}$ ions are calculated at the three energies measured. The errors shown are just the statistical exrors calculated from the data of Table $V$; the probable systematic error is about double the quoted errors. The average charge $\bar{Z}$ and mean square charge $\overline{z^{2}}$ are also listed. (See text pp. 29, 34.)

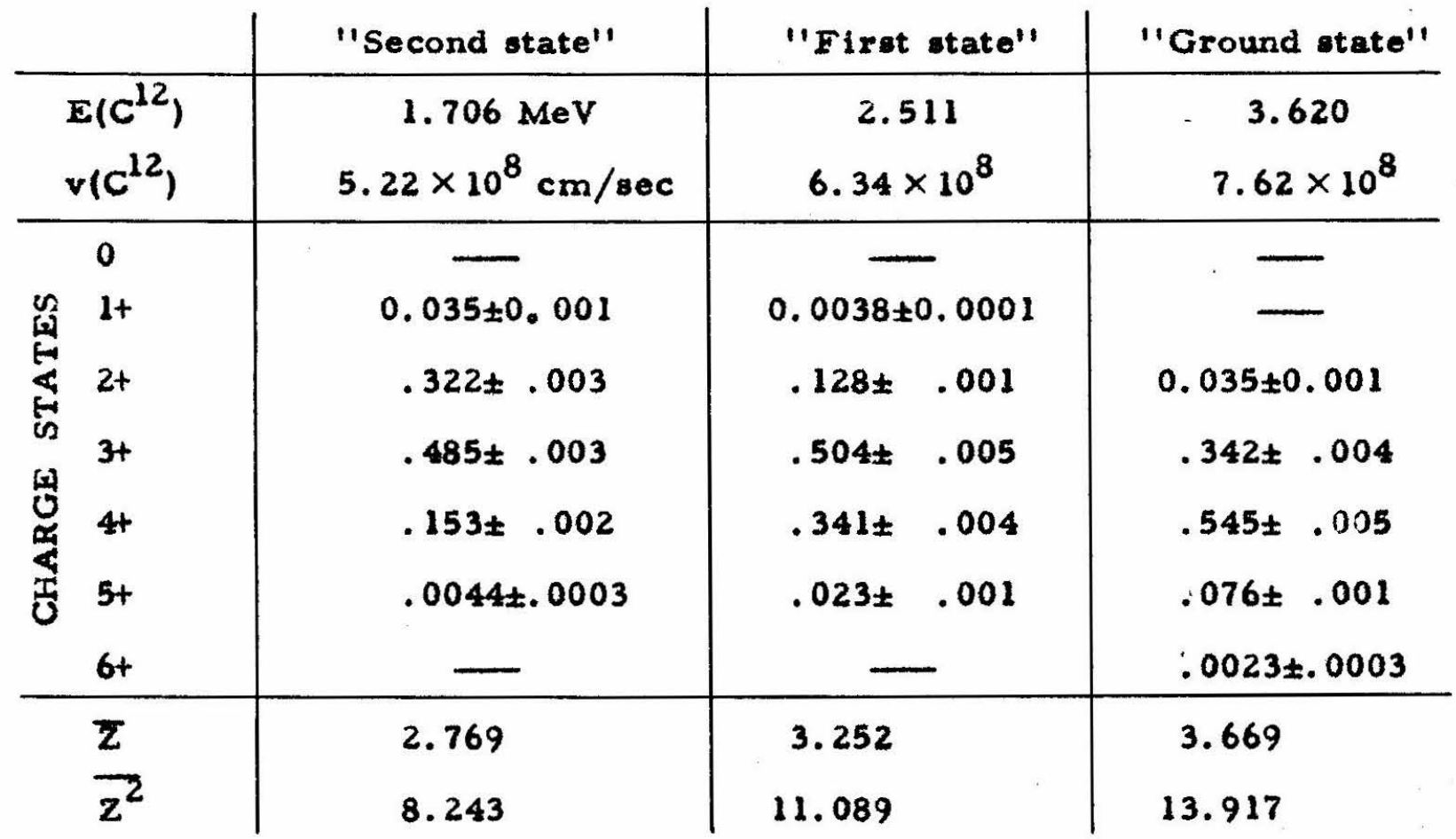




\section{Table VII}

Effective Solid Angle for Coincidence Detection

The effects of $\gamma$-emission, the elliptical aperture of the spectrometer, and the rectangular alphe slit are included; $w$ and in are the width and height of the rectangular slit in inches, and d\& is given in units of $10^{-3}$ steradian. $G$ is the ratio of $d S$ for the second atate to dS for the first state. (See text p. 34.)

\begin{tabular}{ccccc|c}
$w$ & h & \multicolumn{3}{c}{ gs } & \\
\hline $.032^{\prime \prime}$ & $0.200^{\prime \prime}$ & 11.1 & 9.9 & 8.5 & $0.866 \pm 0.028$ \\
.040 & .200 & & 10.8 & 9.7 & $.892 \pm .013$ \\
.050 & .200 & & 11.1 & 10.3 & $.932 \pm .007$ \\
.060 & .200 & & & 10.6 & $.957 \pm .005$ \\
.062 & .245 & & 11.1 & 10.7 & $.959 \pm .005$
\end{tabular}


Table VIII

Thickness Factors

In the firgt part of the table, factora necossary to calculate the stopping power $\epsilon$ for $\mathrm{C}^{12}$ ions in $\mathrm{VN}$ at three energies of interest are listed. $E_{p}$ is the energy of the proton with the same velocity as the $C^{12}$ ion of energy $E\left(C^{12}\right)$, and $\epsilon_{N}$ and $\epsilon_{V}$ are the nitrogen and vanadium stopping powers for the protons (Reynolds 1953, Bader 1956). $\overline{z^{2}}$ is the mean square charge, from Table VI. The second portion of the table then liats the calculated $\Delta \mathrm{E}$ in $\mathrm{keV}$ for various targets. (See text p. 34.) The remaining two sections give the kinematic broadening $\Delta E_{\delta}$ and the broadening due to $y$-emisaion, $\Delta E_{\gamma}$. (See text p. 35.) 
$-66-$

Table VIII

Thickness Factors

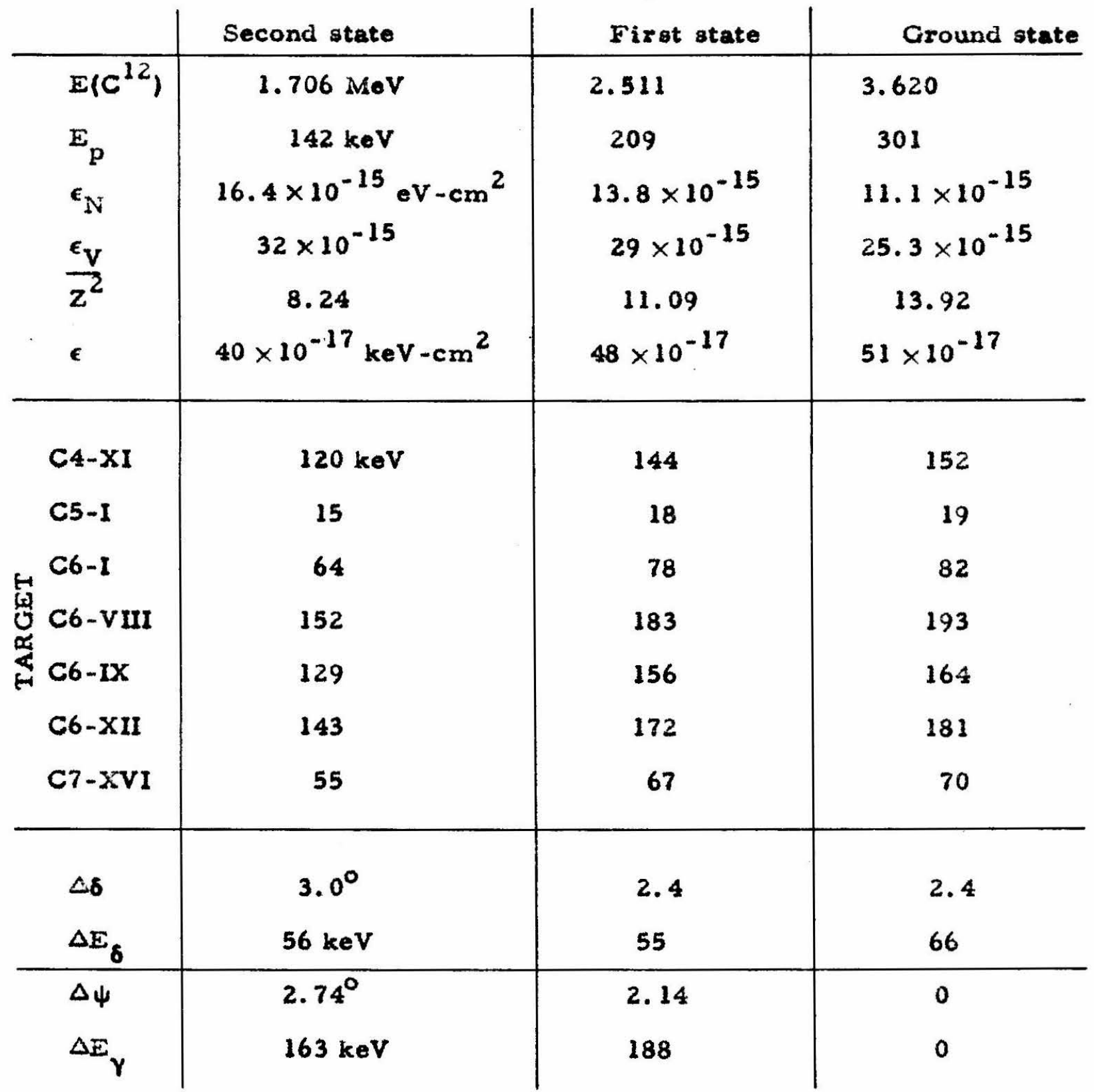


Table $\mathrm{X}$

Morsentum Widths of Recoil-Ion Croups

Calculated (calc.) and observed experimental (exp.) values of $\Delta_{p} / p$ for various targets and energies are given. Calculated values are the square root of the sum of the squares of the terms from Table VIII. (See text p. 35.)

\begin{tabular}{|c|c|c|c|c|c|}
\hline \multirow[t]{2}{*}{ Target } & \multirow{2}{*}{$\begin{array}{l}\text { Second State } \\
\text { calc. }\end{array}$} & \multicolumn{2}{|c|}{ First State } & \multicolumn{2}{|c|}{ Ground State } \\
\hline & & calc. & exp. & calc. & exp. \\
\hline C4-XI & 0.062 & 0.048 & 0.060 & 0.023 & $\left\{\begin{array}{r}0.032 \\
.026\end{array}\right.$ \\
\hline C5 -I & .051 & .039 & .041 & .010 & \\
\hline C6-I & .054 & .042 & .039 & .017 & .020 \\
\hline C6-VIII & .067 & .053 & $\left\{\begin{array}{l}.049 \\
.050\end{array}\right.$ & .028 & \\
\hline C6-IX & .063 & .050 & & .024 & \\
\hline C6-XII & .066 & .052 & & .027 & \\
\hline C7-XVI & .053 & .041 & & .013 & \\
\hline
\end{tabular}


$-68-$

Table $\mathrm{x}$

Thickness Tactor $T$

The fraction of the momentum width of recoil-ion groups from second and first state reactions which lie within the $5 \%$ momentum window of the spectrometer are listed. Widths are the calculated $\Delta p / p$ values from Table IX. $T$ is the ratio of the fraction of second state to the fraction of first otate within the window. The error quoted are estimated probable errors. (See text p. 36.)

\begin{tabular}{l|c|c|c} 
Target & Second State & Firet State & \multicolumn{1}{c}{ I } \\
\hline C6-I & 0.93 & 1.00 & $0.93 \pm .06$ \\
C6-VII & .75 & .94 & $.80 \pm .08$ \\
C6-IX & .79 & 1.00 & $.79 \pm .08$ \\
C6-XII & .76 & .96 & $.79 \pm .08$ \\
C7-XVI & .94 & 1.00 & $.94 \pm .05$
\end{tabular}


Table XI

Calculation of Weighting Eactors W

The weighting factors $W$ are calculated by equation 16 for the data of Table IV, using $P=3.00 \pm .09, C=1.421 \pm .036, C$ from Table VII, and $T$ from Table $X$. (See text p. 36.)

\begin{tabular}{|c|c|c|c|c|c|c|}
\hline Run & Target & $\alpha$ & $R_{1}$ & $a$ & $T$ & $w \times 10^{-4}$ \\
\hline 1 & C6-IX & 332 & 420 & 0.866 & 0.79 & $4.52 \pm .67$ \\
\hline 2 & & 94.8 & 370 & & & $1.14 \pm .14$ \\
\hline 3 & C6-XII & 165.0 & 420 & & .79 & $2.24 \pm .33$ \\
\hline 4 & C7-XVI & 111.4 & 460 & & .94 & $1.98 \pm .17$ \\
\hline 5 & & 348.3 & 530 & & & $7.12 \pm .62$ \\
\hline 6 & & 94.9 & 510 & & & $1.87 \pm .16$ \\
\hline 7 & & 34.5 & 310 & .959 & & $0.46 \pm .04$ \\
\hline 8 & & 162.3 & 349 & .957 & & 2. $35 \pm .16$ \\
\hline 9 & C6-I & 422.1 & 462 & & .93 & $8.19 \pm .71$ \\
\hline 10 & & 225.9 & 450 & & & $4.29 \pm .45$ \\
\hline 11 & & 279.0 & 365 & & & $4.30 \pm .44$ \\
\hline 12 & C6-VIII & 424.8 & 325 & & .80 & $5.01 \pm .62$ \\
\hline 13 & & 421.3 & 380 & & & $5.81 \pm .67$ \\
\hline 14 & & 501.5 & 370 & & & $6.74 \pm .81$ \\
\hline 15 & & 385.3 & 305 & & & $4.26 \pm .45$ \\
\hline
\end{tabular}


Table XII

Calculation of $L_{\mathrm{rad}} / \mathrm{L}$

The weighting factor W (from $T$ able XI) and Cousts (from Table IV) are summed for each target, and $\mathrm{I}_{\mathrm{rad}} / \mathrm{I}$ is calculated by equation 16. External exror is calculated from the deviations $\Delta$ of each value from the mean, and internal error is calculated from the erros of $2(W)$ and $z$ (Counto). (See text p. 36.)

\begin{tabular}{l|c|c|c|cc} 
Target & Counts & $\forall \times 10^{-4}$ & $\left(I_{\mathrm{rad}} / \mathrm{x}\right) \times 10^{4}$ & $\Delta$ & \multicolumn{1}{|c}{$\Delta^{2}$} \\
\hline C6-IX & 12 & $5.66 \pm .68$ & $2.12 \pm .66$ & -0.70 & 2.77 \\
C6-XII & 10 & $2.24 \pm .33$ & $4.46 \pm 1.56$ & +1.64 & 6.02 \\
C7-XVI & 43 & $13.78 \pm .68$ & $3.12 \pm .50$ & +0.30 & 1.24 \\
C6-I & 52 & $16.78 \pm .95$ & $3.10 \pm .47$ & +0.20 & 1.31 \\
C6-VIII & 53 & $21.82 \pm 1.30$ & $2.43 \pm .37$ & -0.39 & 3.32 \\
\hline$\Sigma$ & 170 & $60.28 \pm 1.91$ & $2.82 \pm .23$ (internal) &
\end{tabular}


$-71 A-$

Figure 1

Energy Level Diagram of $\mathrm{c}^{12}$

The level of interest is the $7.656-\mathrm{MeV}$ level. The drawing is from Lauritsen and Ajzenberg-Selove (1962); for explanation of conventions followed see Ajzenberg-Selove and Lauritsen (1959). (See text pp. 2, 4, 5, 32.) 


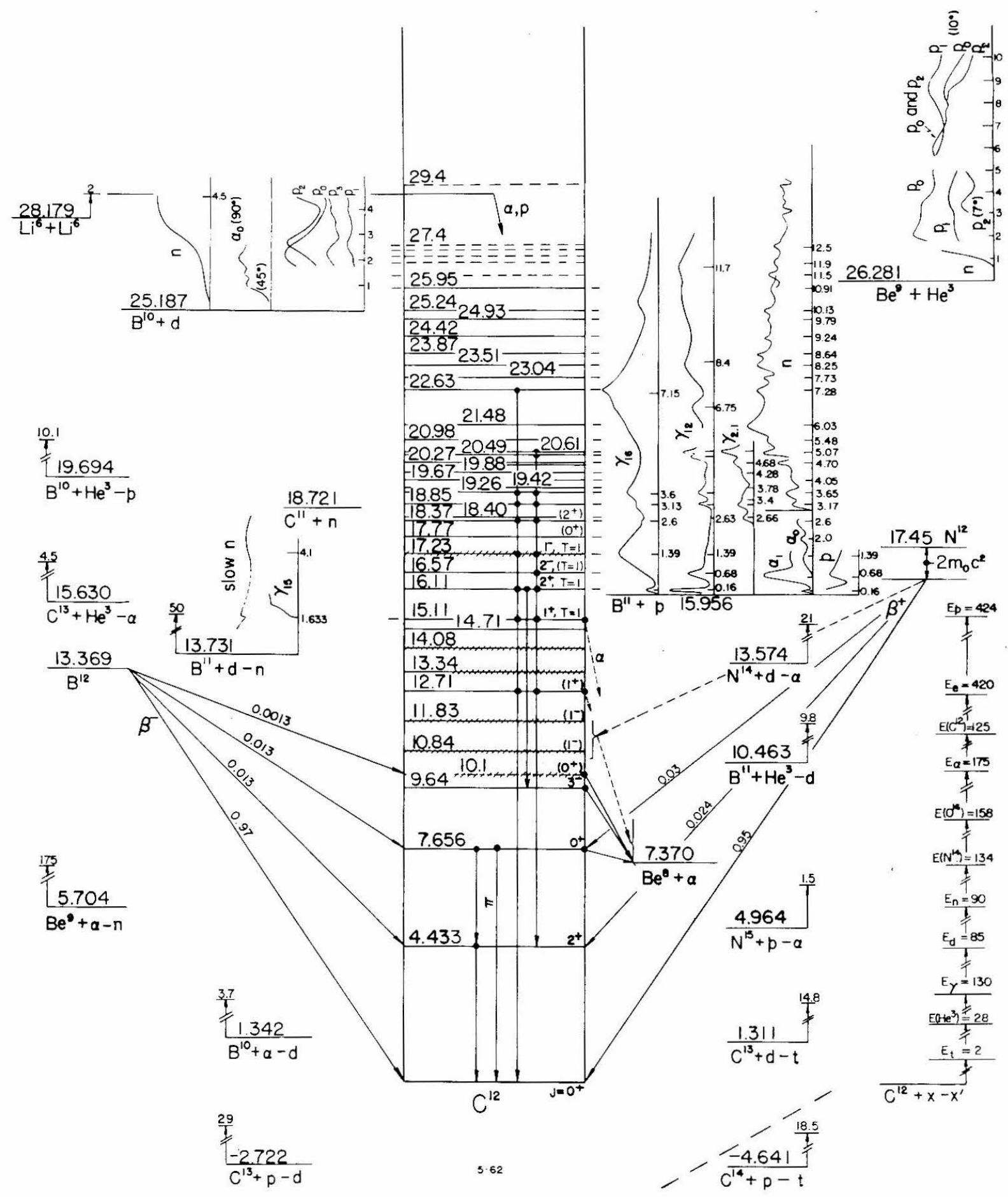

FIGURE I. 
Eigure 2

Plan of Experiment

The arrangement of the equipment used in the experiment is shown. Recoil $\mathrm{C}^{12}$ ions at $90^{\circ}$ were analyzed and detected, and the pulses amplified; alpha pulses from a counter in the target chamber were delayed so as to coincide with the $\mathrm{C}^{12}$ pulses; and whenever a colncidence occurred the two pulses were displayed on the dual-beam oscilloscope and photographed. (See text pp. 5-15; recoil-ion detection p. 7, alpha detection p. 9, coincidence detection p. 12.) 
PLAN OF EXPERIMENT

ALTERNATING GRADIENT SPECTROMETER

$C^{12}$

DETECTOR

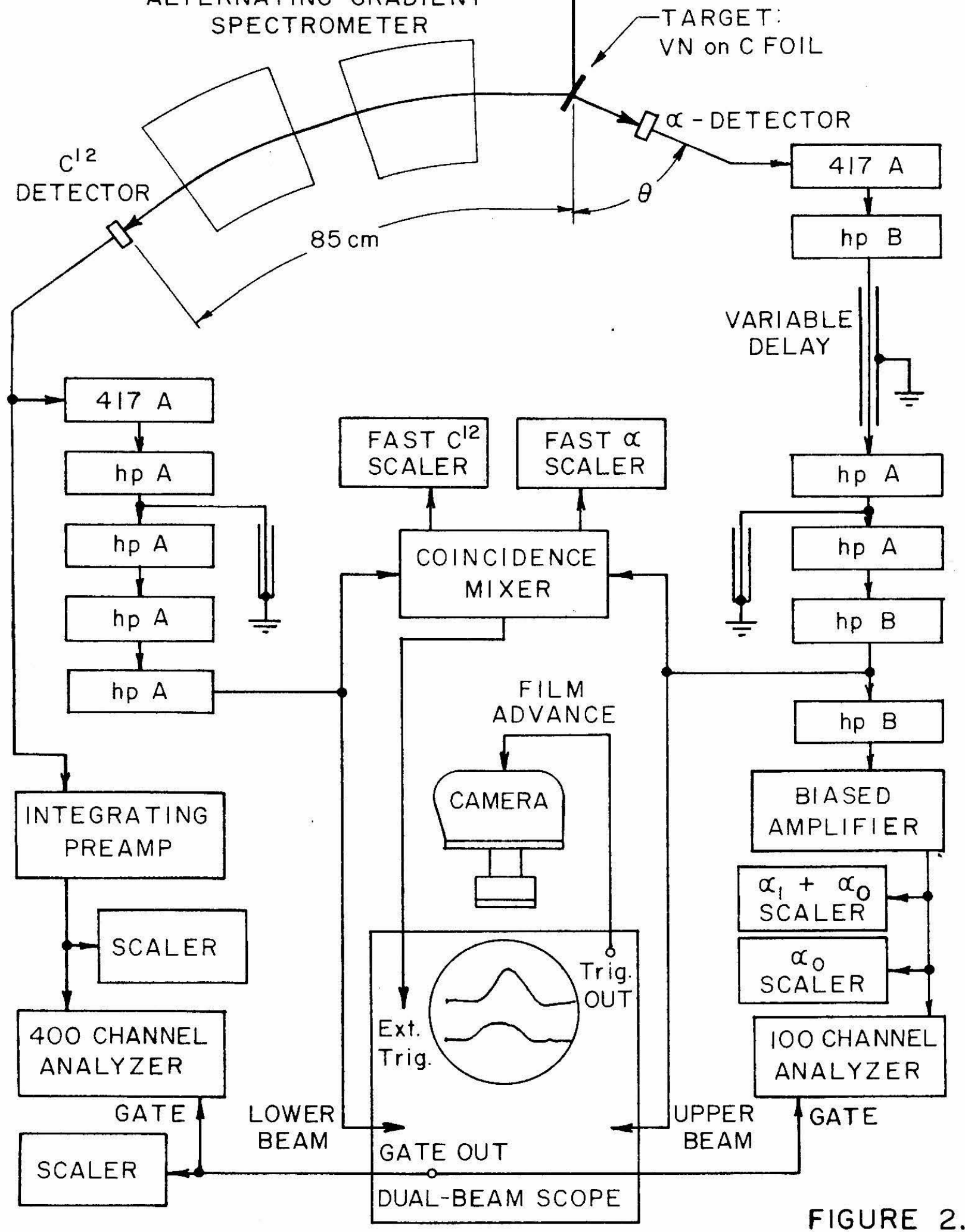

INCIDENT BEAM

I. $8 \mathrm{MeV} d$ 
Figure 3

Nitriding Chamber

Targets were nitrided by heating in an ammonia atmosphere. The heating was accomplished by bombardment with an alpha-particle beam at the straight-through port of the accelerator. Ammonia was admitted from the stainless-steel flask cooled to dry ice temperature; flow was controlled by needle valye. The efficiency of the differential pumping and cold trap arrangement shown was such that an ammonia pressure of $1 \mathrm{~mm} \mathrm{Hg}$ read on the thermocouple gauge caused no increase in the pressure reading of the accelerator column. Note that the beam passes through a length of copper tubing soldered to a copper rod leading to the cold trap. (See text p. 7, Appendix A.) 


\section{NITRIDING CHAMBER}

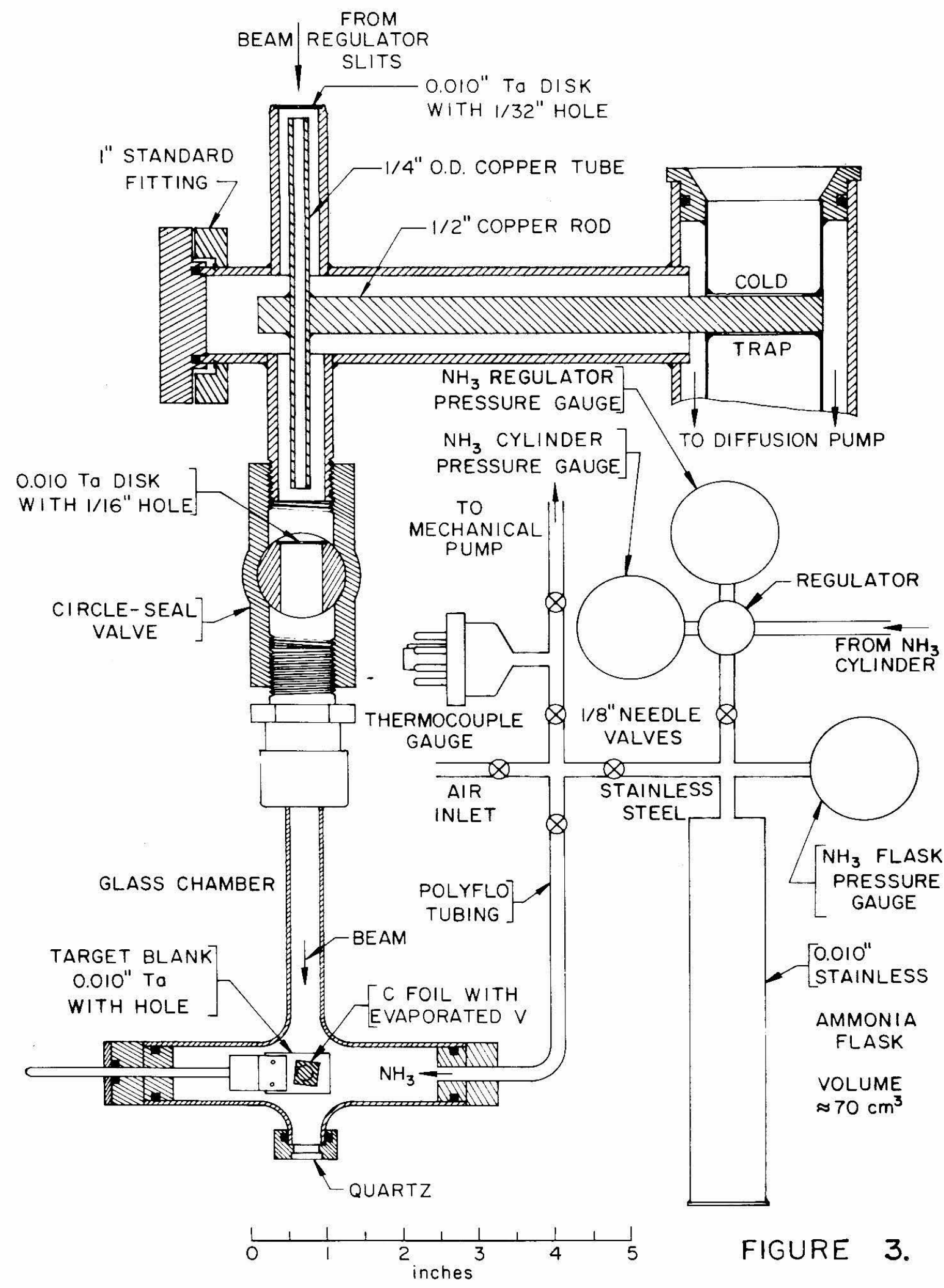




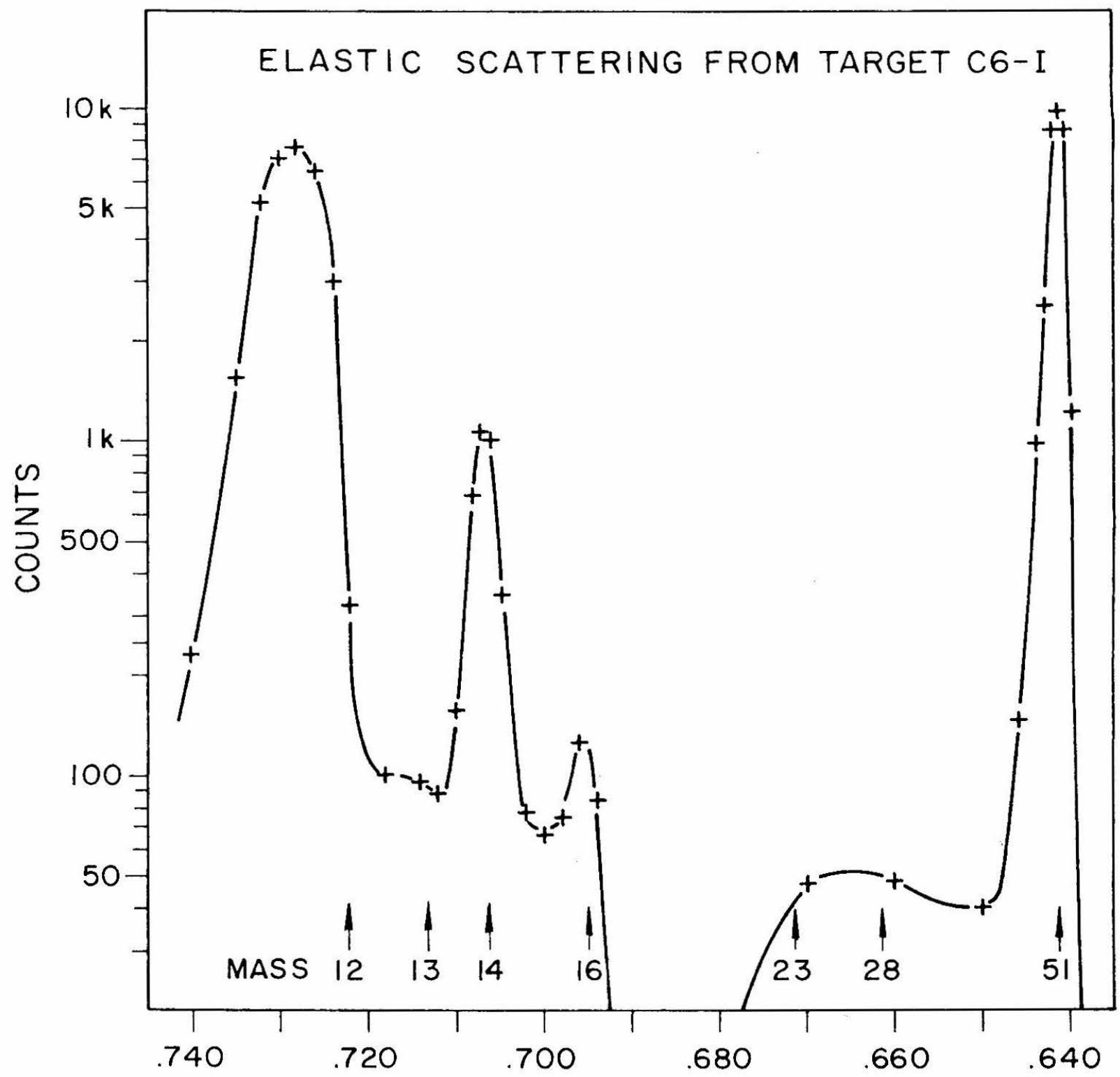

FLUXMETER I (volts)

Elastic Scattering from Target C6-I

FIGURE 4.

The momentum spectrum of elastically scattered protons of $1-\mathrm{MeV}$ incident energy, as measured with a $10 \frac{1}{2}$-inch magnetic spectrometer at a laboratory angle of $150^{\circ}$, is shown. The arrows indicate the locations of groups scattered from various masses, normalized to the $\mathrm{V}^{51}$ peak. (See text p. 7, Appendix A.) 


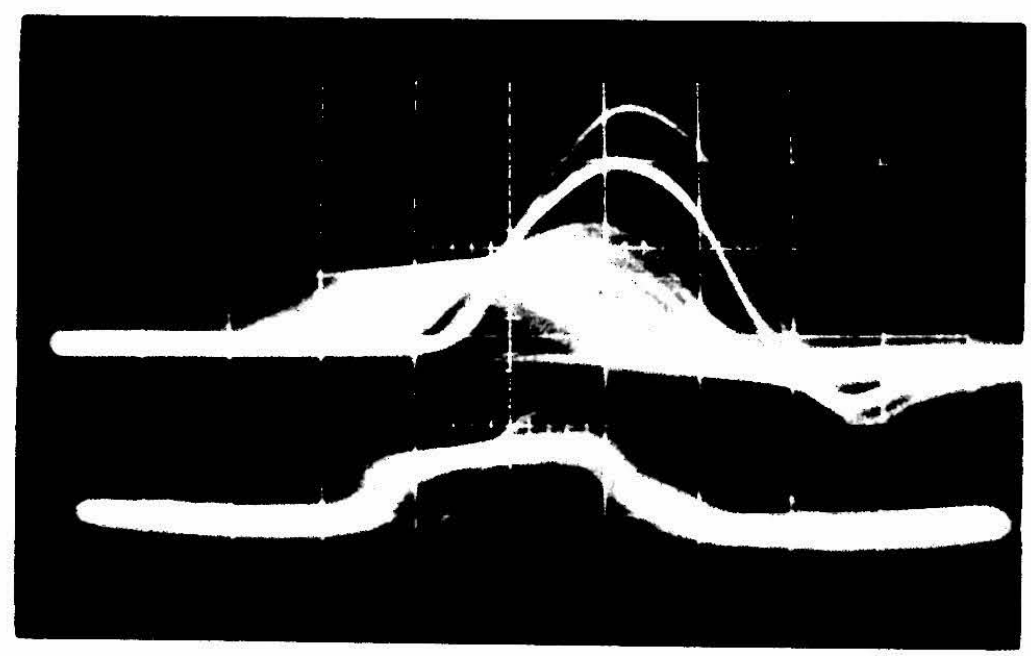

FIGURE $5 a$.

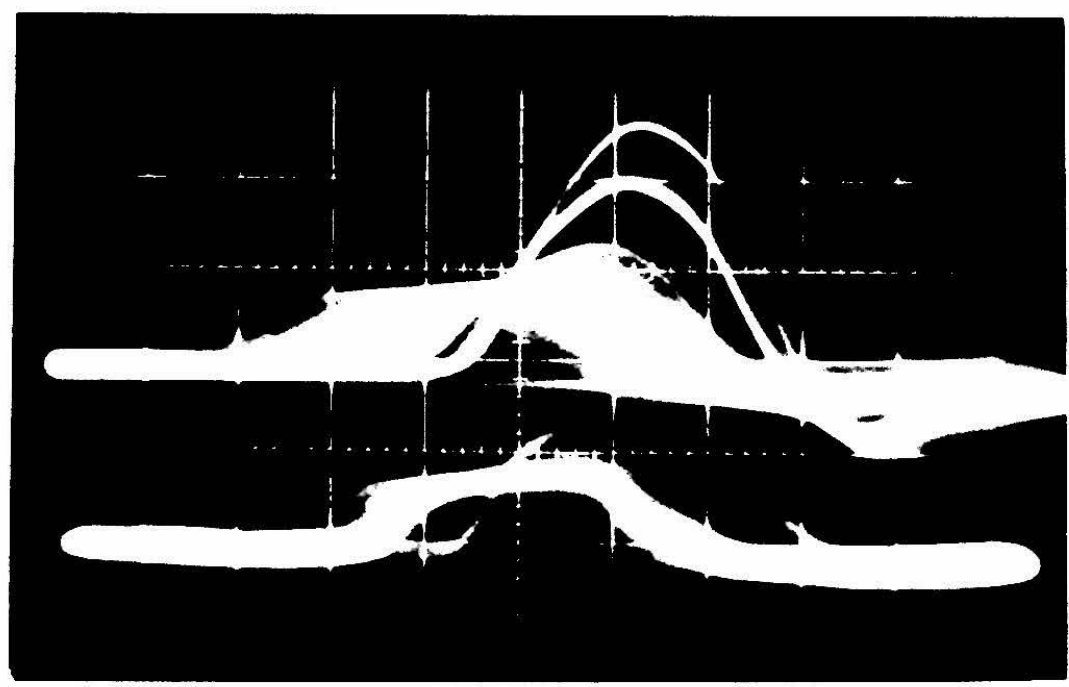

Non-Coincident Spectra

FIGURE $5 \mathrm{~b}$.

Thesc two prints (from the same negative) show the non-coincident alpha spectrum on the upper beam and the spectrum at the magnet focus on the lower beam of the dual-beam oscilloscope. (See text pp. 9, 11, 19.) Figure 5i: show s barrel distortion as it occurs on the negative; in Figure $5 \mathrm{~b}$ the distortion has been removed by using the camera lens as an enli.rger. (See text p. 15, Appendix B.) 
$-76 A-$

Figure 6

Variable Angle Target Chamber

The counter angle $\theta$ in this chamber is continuously variable from $35^{\circ}$ to $90^{\circ}$. The "rectangular" slit, mounted in the counter holder $5 / 8$ inch from the center of the chamber, determines the solid angle of the detector; slits of various sizes were made. For details of the chamber, see Kellogg drawing \#1B2-75. (See text p. 9.) 


\section{VARIABLE ANGLE TARGET CHAMBER}

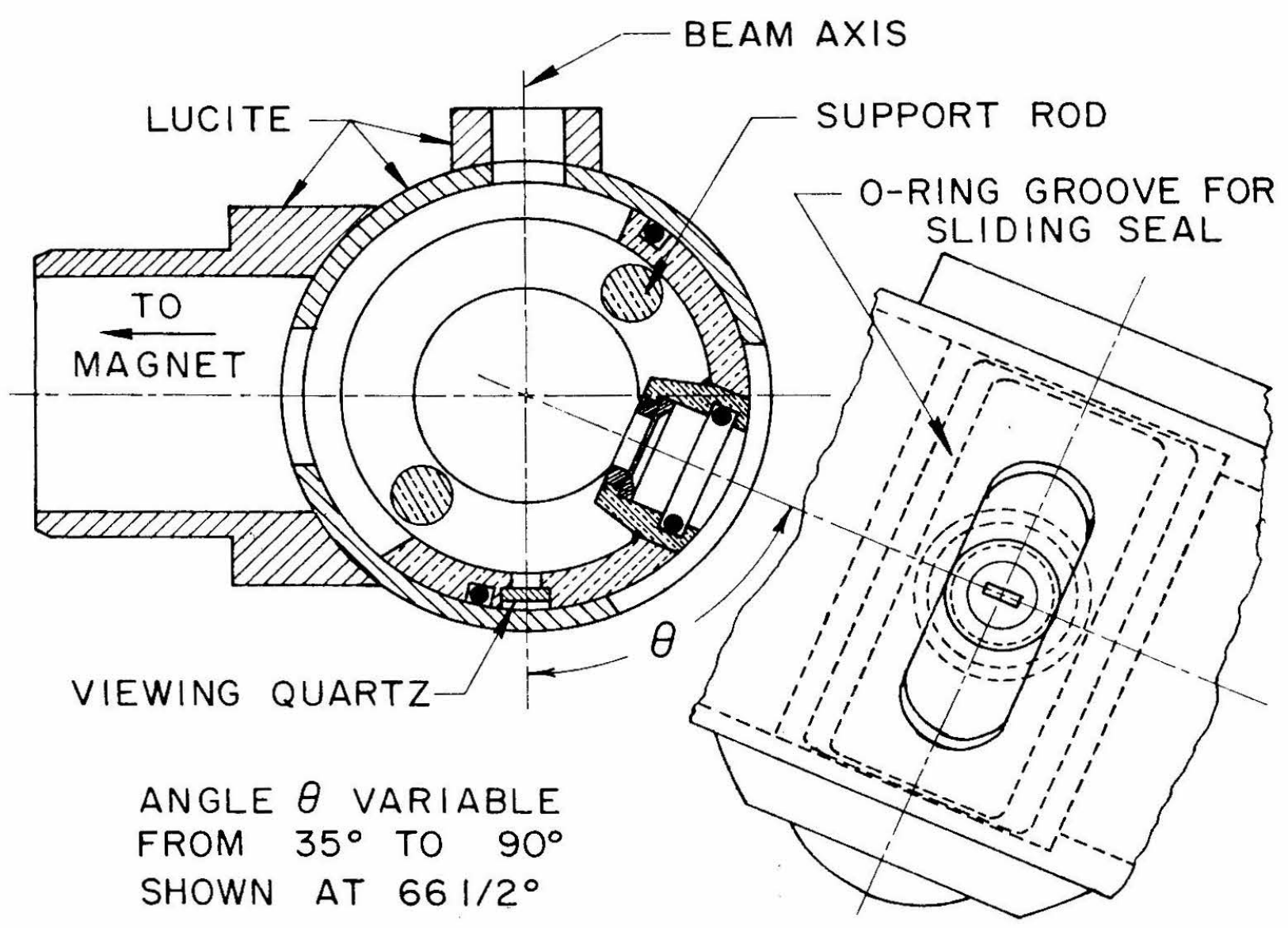

FULL SIZE

SEE DRAWING \# IB2-75

"RECTANGULAR" SLIT DETAIL

2 I/2 TIMES SIZE

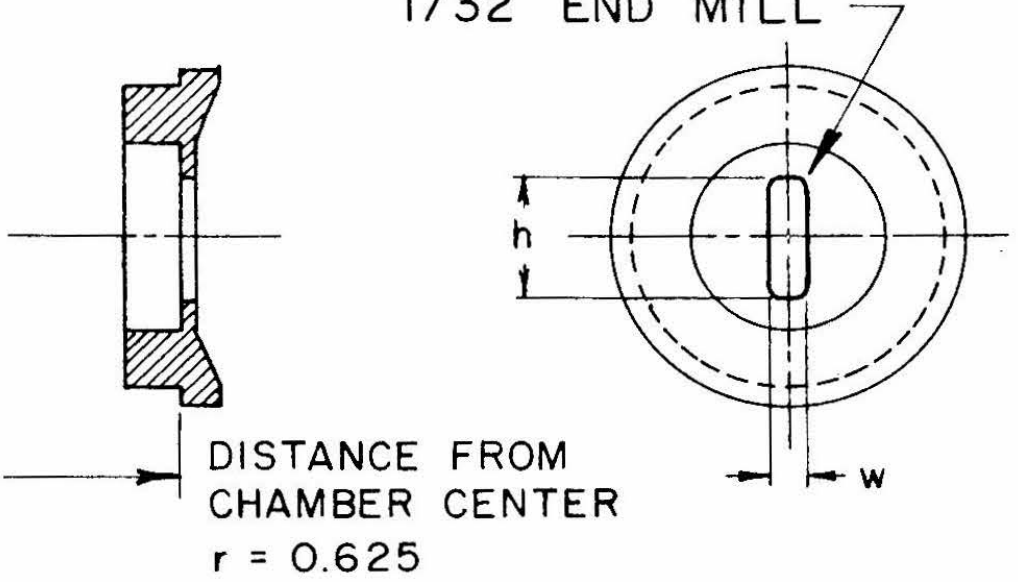

\section{$1 / 32^{\prime \prime}$ END MILL}

\begin{tabular}{c|c}
$w$ & $h$ \\
\hline .032 & .200
\end{tabular} .040 .200 .050 .200 .060 .200 $.062, .245$

FIGURE 6. 


\section{COUNTER MOUNT \\ FOR VARIABLE $\theta$ CHAMBER}

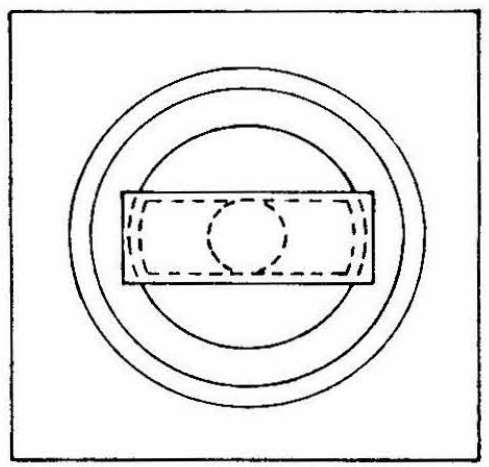

GOLD EVAPORATED

OVER ENTIRE

FRONT SURFACE

FILL WITH EPOXY

\section{(1)}
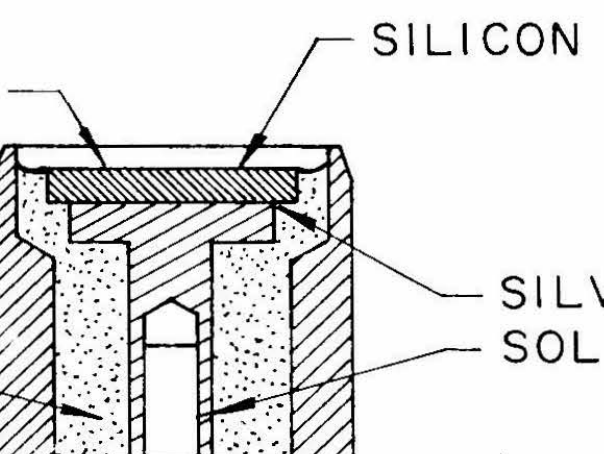

- SILVER PASTE SOLDER

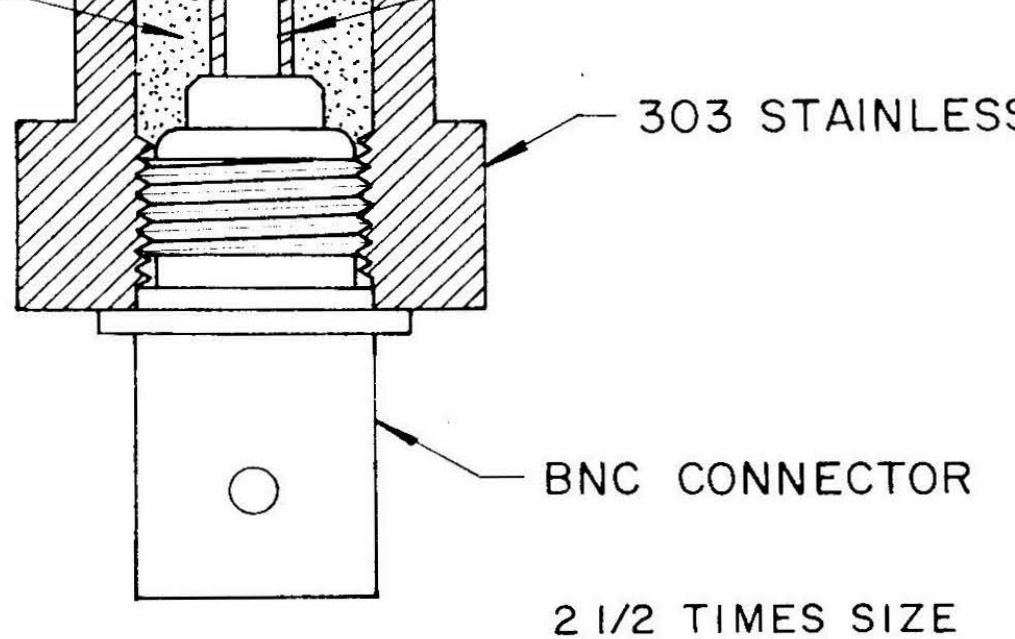

FIGURE 7.

Counter Mount for Variable Angle Chamber.

The gold evaporated onto the silicon to make a rectifying junction also makes contact with the grouncied cass. The signal lead makes ohmic contact to the rear surface of the silicon. (See text p. 10.) 


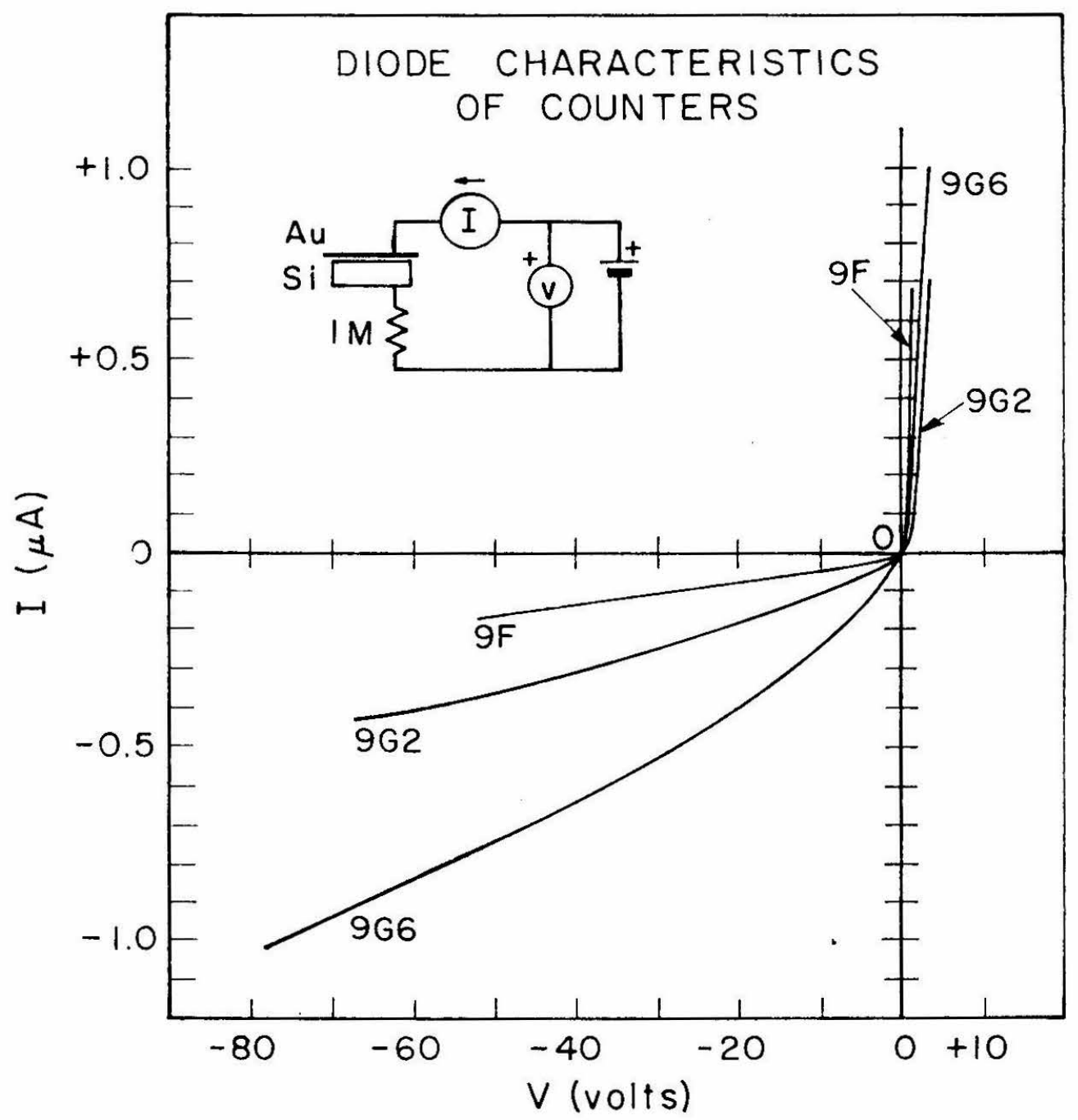

FIGURE 8.

Diode Characteristics of Counters

The forward and reverse current-voltage characteristic of gold-silicon surface-barrier counters was measured as indicated in the figure. The three urits displayed here, made with $900 \Omega-\mathrm{cm}$ silicon, were all successful courters. In use, a reverse bias of about 50 volts was applied. (See text p. 10.) 


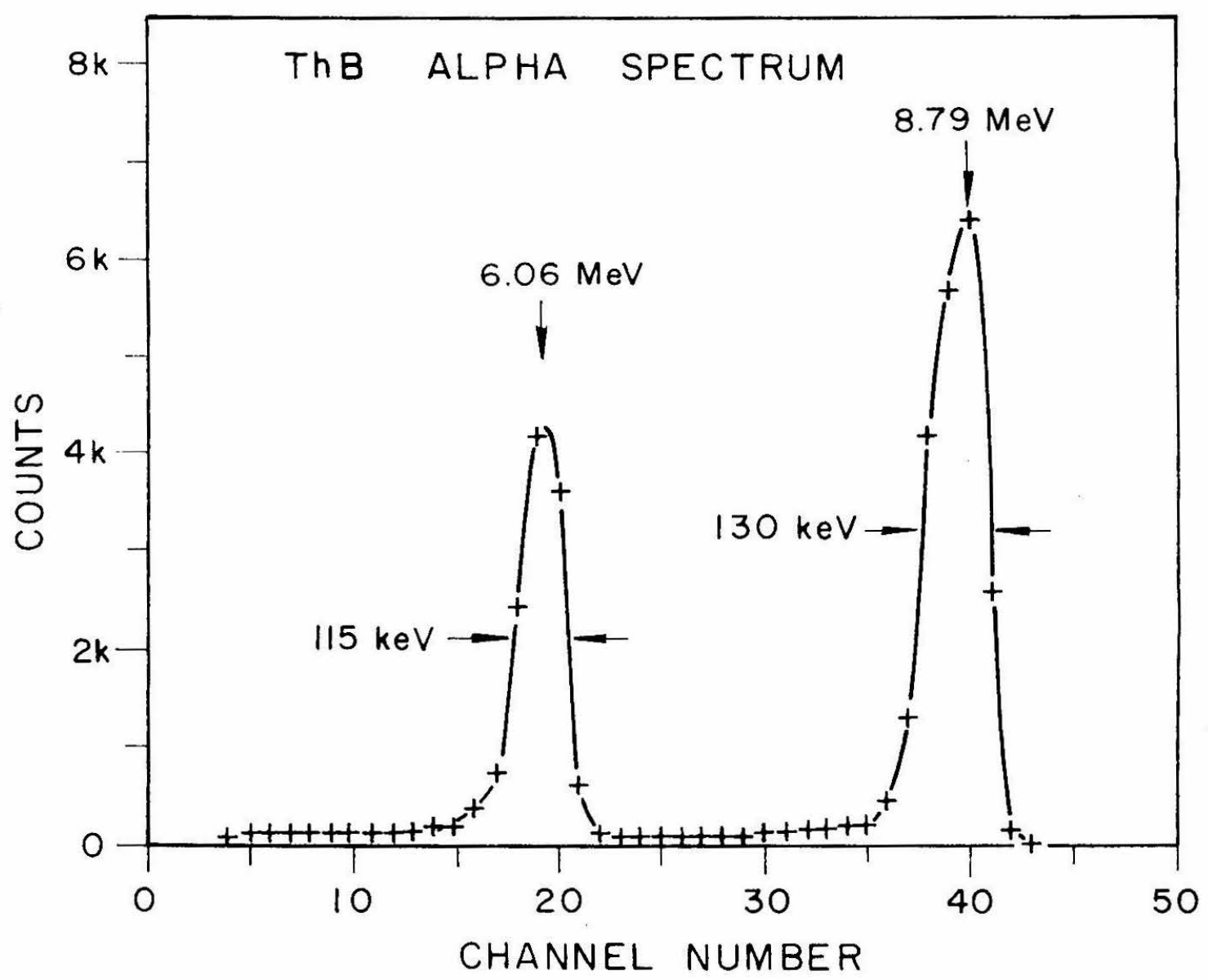

FIGURE 9.

ThB Alpha Spectrum

This spectrum was taken $\mathrm{v}$ ith counter $9 \mathrm{~F}$ and the alpha-channel electronics of Figure 2. The amplifier bias corresponds to $3.52 \mathrm{MeV}$. (See text pp.10,17.) 
Eigure 10

Coincidence Mixer

Tunnel diodes are used at both inputs of the mixer as discriminators, and a tunnel dlode is also used for the mixer. The output for a pulse at either input sufficient to trigger the diode is a $20-\mathrm{V}, 2-\mu \mathrm{sec}$ pulse. Note that the coincidence-pulse output was from point B, with a $220-0 h m$ plate load on the output tube. This pulse was used to trigger the oscilloscope swoep. (See text p. 12.) 
$-80-$

COINCIDENCE MIXER
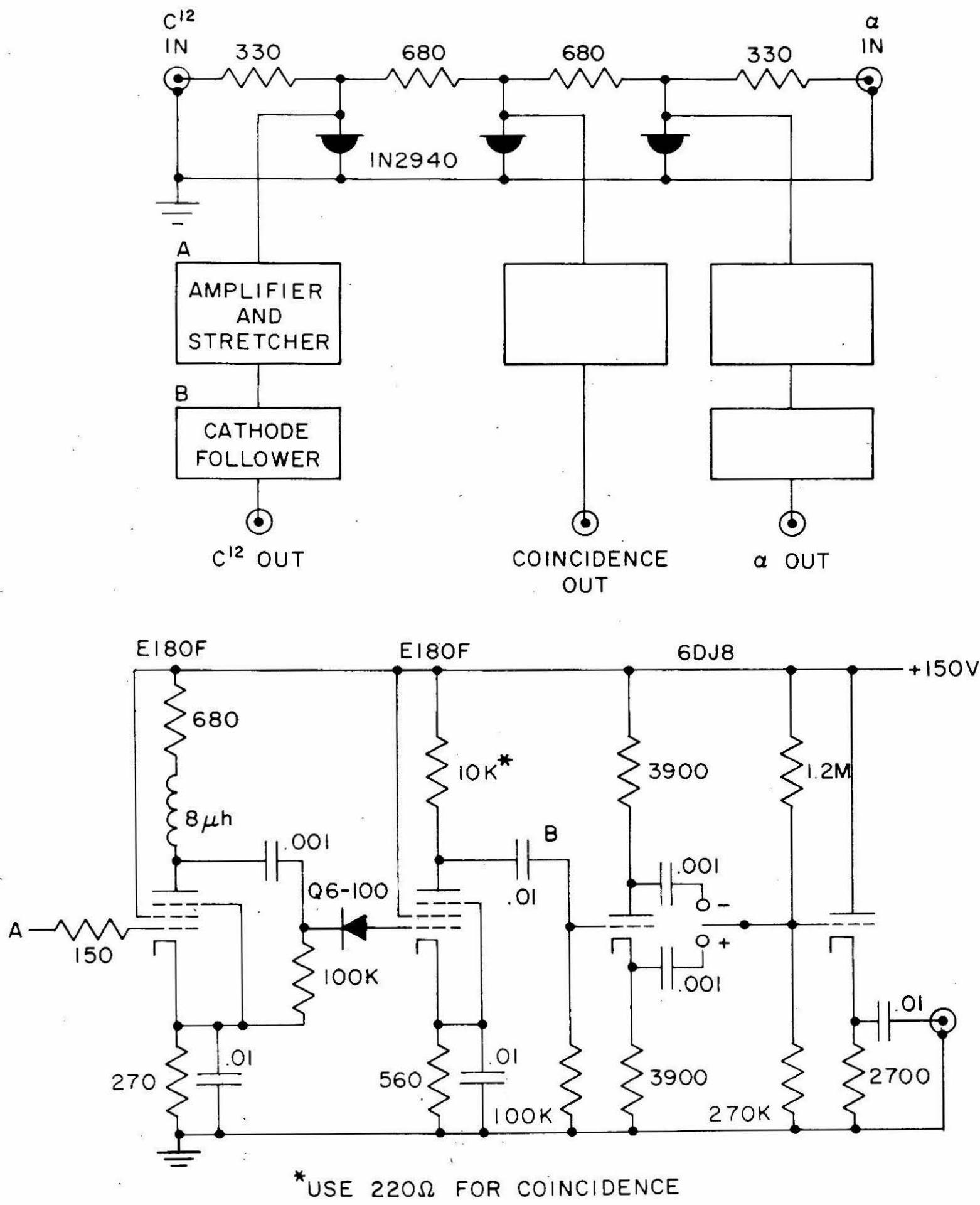

FIGURE 10. 
$-81 A-$

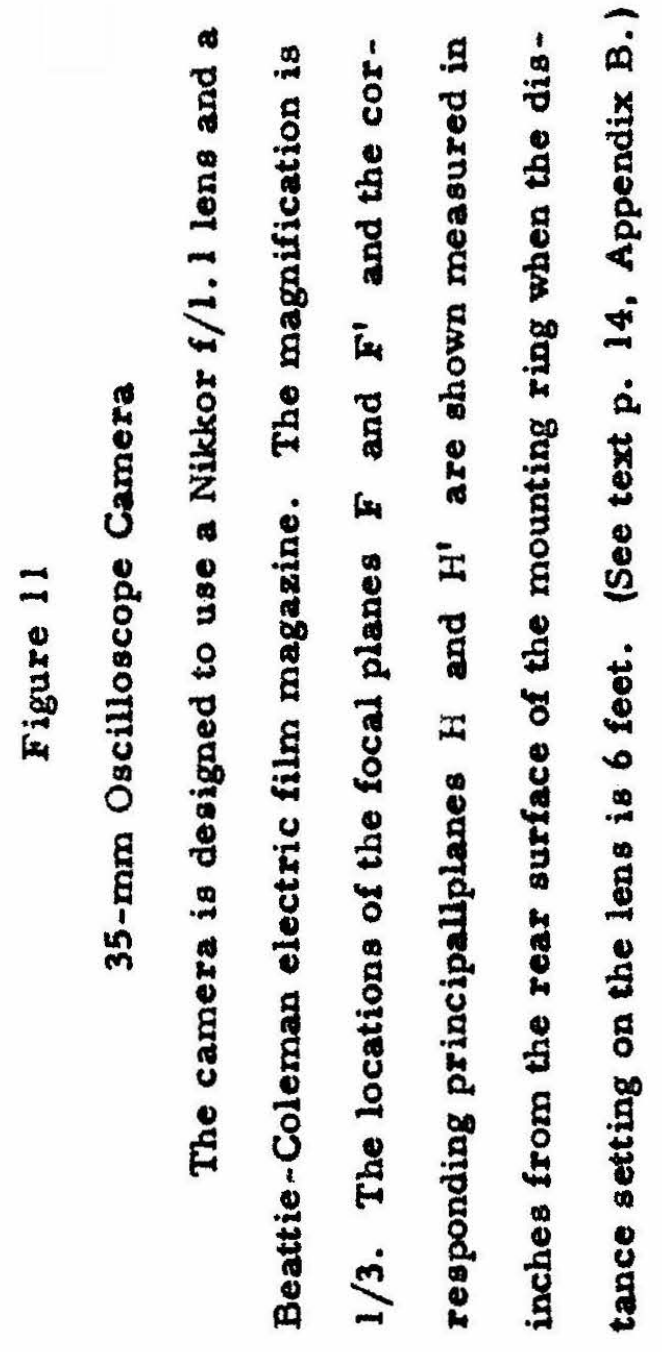


$-81-$

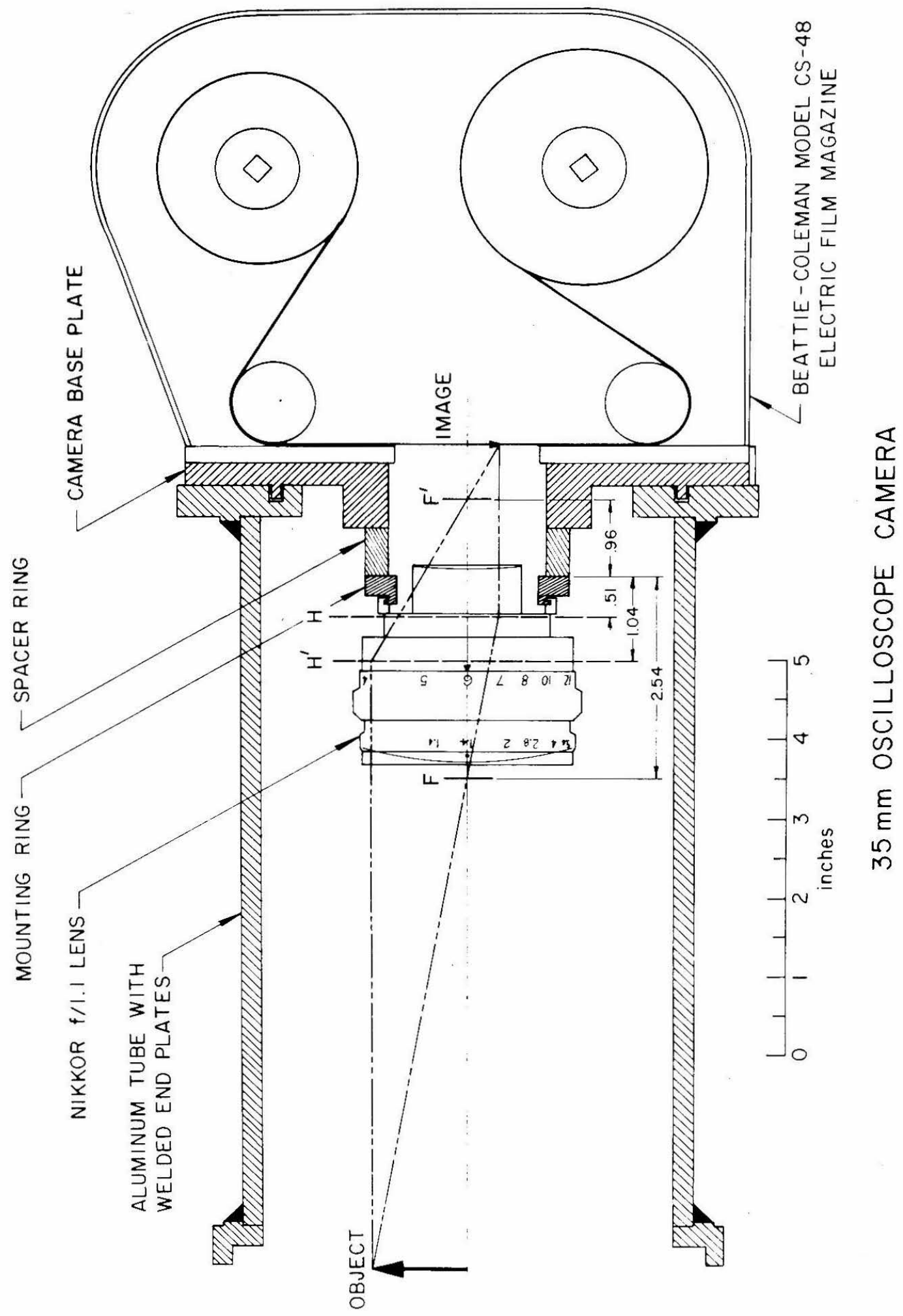

$=$
$=$
$\frac{w}{2}$
$\frac{0}{u}$ 
$-82 A-$

Figure 12

Film-Advance Circuit

A suitable pulse (e.g. $8-\mathrm{V}, 0.6-\mu s e c)$ at the "trigger in" switches the silicon controlled rectifier (SCR) to it 3 conducting mode, and current through the $10 \mathrm{~K}$ resistor holds it conducting while the current in the relay coil builds up. When the relay closes, current is supplied to the film-advance motor. When the film has moved far enough to open the cam-operated microswitch, the relay opens and the SCR is turned off; current is then supplied to the motor through the microswitch until the film has advanced one frame. The relay is also used to operate a Sodeco counter which is used to count frames on the film. (See text p. 15, Appendix B.) 
$-82-$

FILM-ADVANCE CIRCUIT
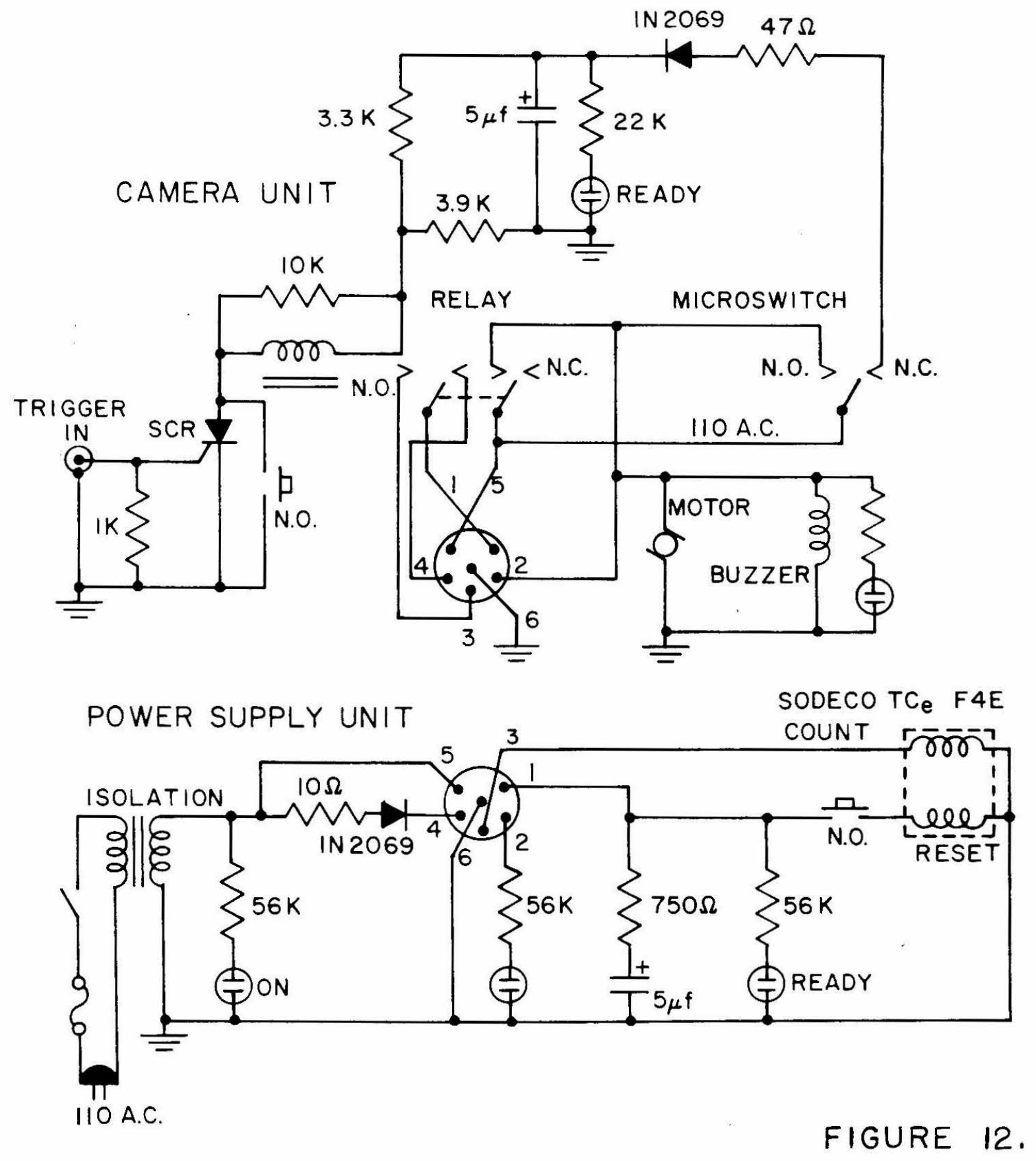


\section{Figure 13}

Energy Spectrum at Spectrometer Focus

In addition to elastically scattered deuterons and reaction products (p and $Q$ ), three recoil-nucleus groups appear in this spectrum, taken with a solid-state counter at the focus of the alternating-gradient spectrometer. The spectrometer momentum resolution was $1.3 \%$, the fluxmeter setting was $1 / I^{2}=16.5$, and the spectrum is normalized to 20,000 alphas observed in the chamber counter. (See text p. 16.) 


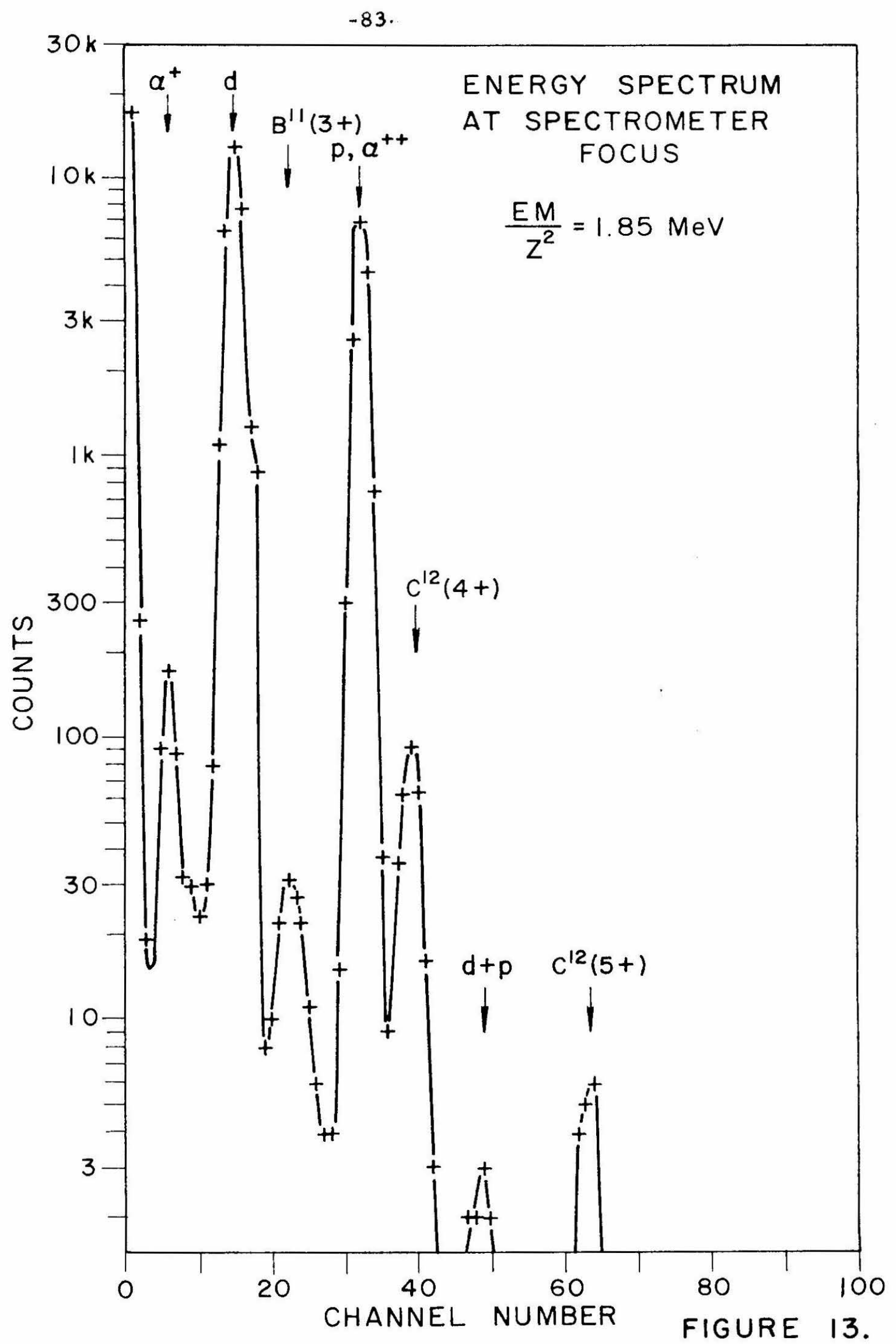




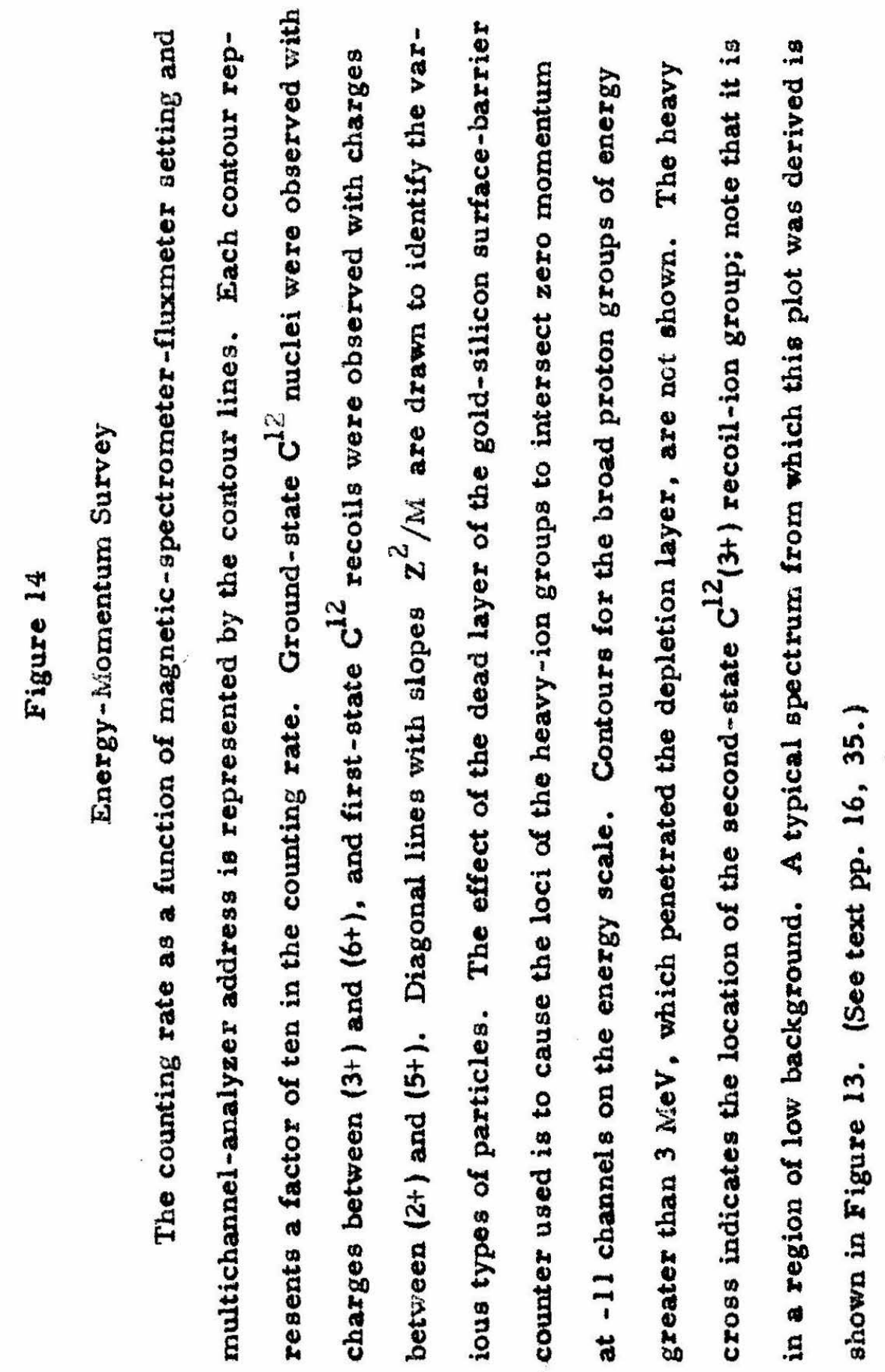


$-84-$

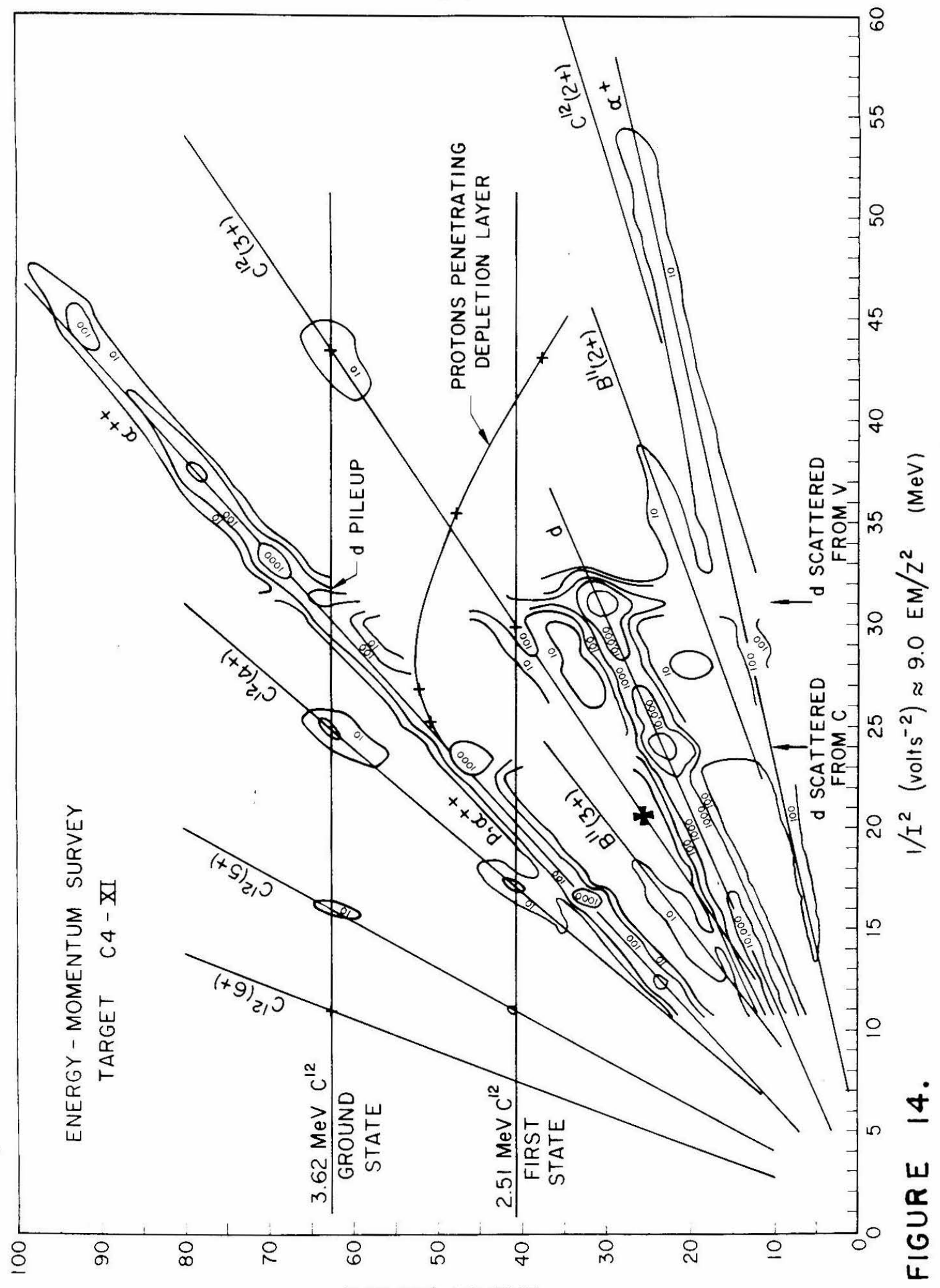

(s|วUนDบว) ᄉอบ $\exists N \exists$ 
$-85-$

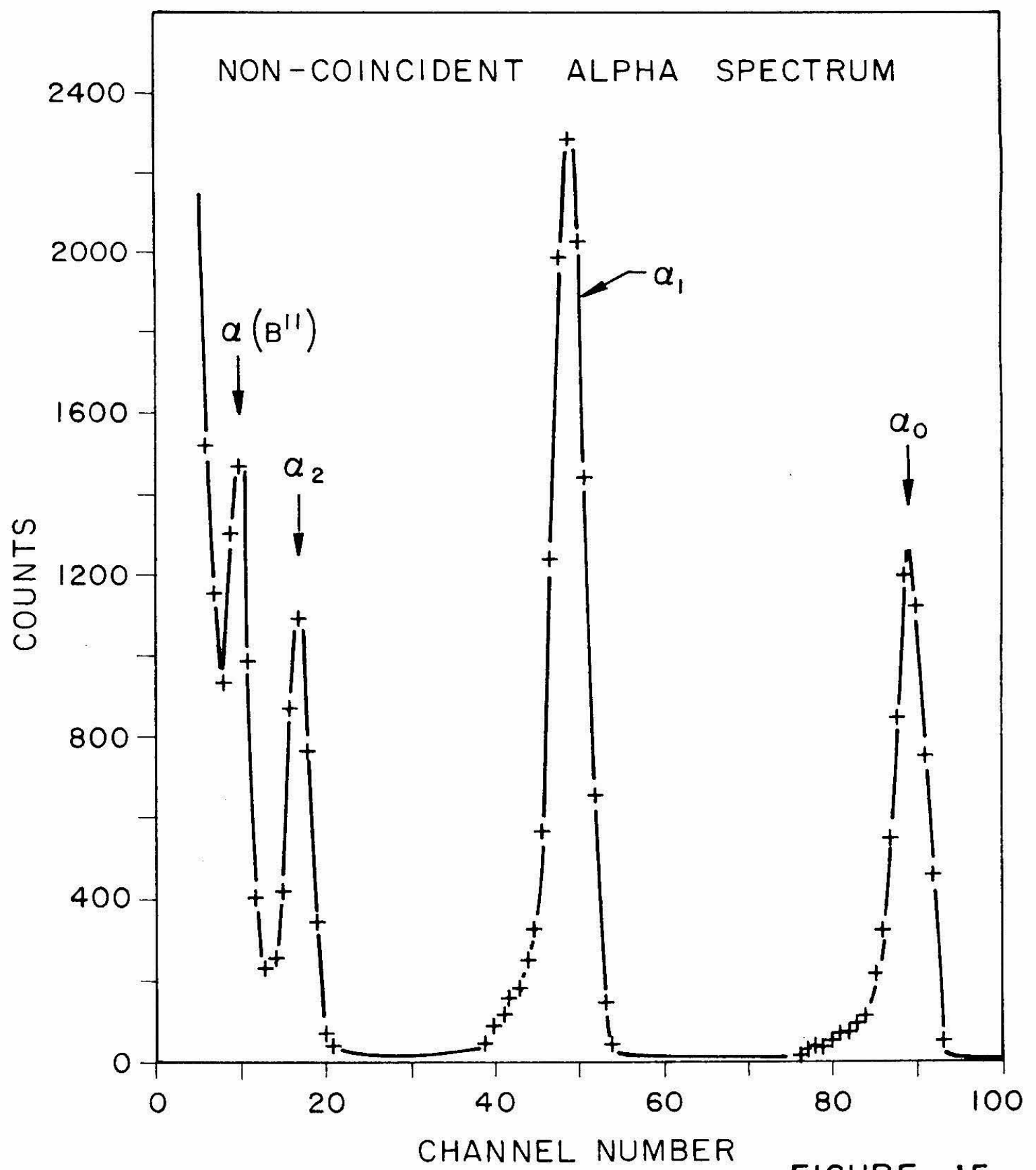

FIGURE 15.

Non-Coincident Alpha Spectrum

This alpha spectrum from the reaction $\mathrm{N}^{14}(\mathrm{~d}, \alpha) \mathrm{C}^{12}$ was taken with the electronics of Figure 2. The counter angle was $67^{\circ}(1 \mathrm{ab})$, and the deuteron bombarding energy $\$$ as $1.800 \mathrm{MeV}$. The bias level of the biased amplifier was about $4 \mathrm{MeV}$. (See text pp. 17, 18, 28.) 


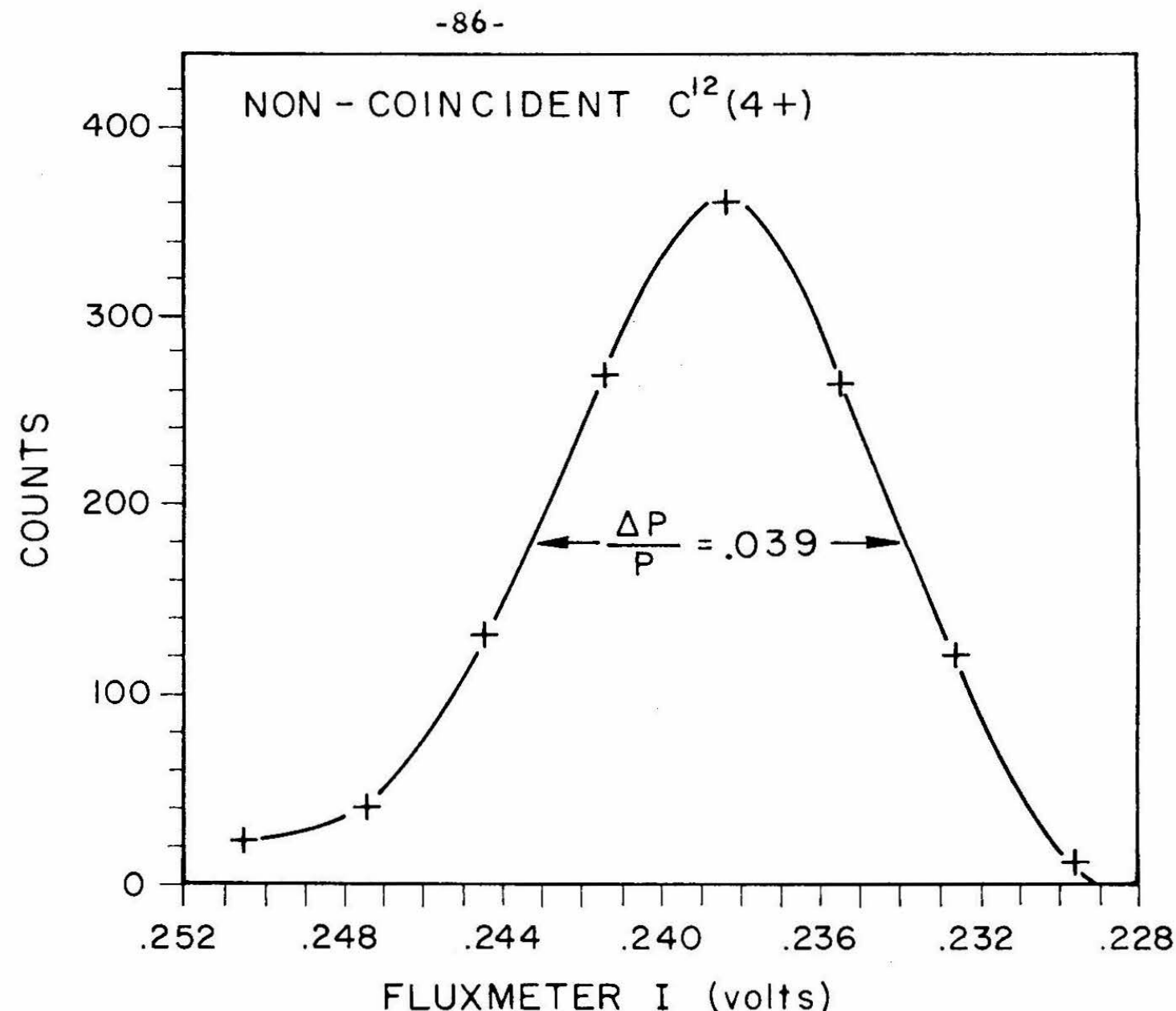

FIGURE 16.

$$
\text { Nor }- \text { - Coincident } \mathrm{C}^{12}(4+)
$$

The spectrometer current was varied to find the maximum $\mathrm{C}^{12}(4+)$ counting rate. The $1.3 \%$ momentum resolution slit was used. This is also a measurement of the momentum width of the recoil-ion group; target C6-I was used for the se data. (See text pp. 17, 35.) 


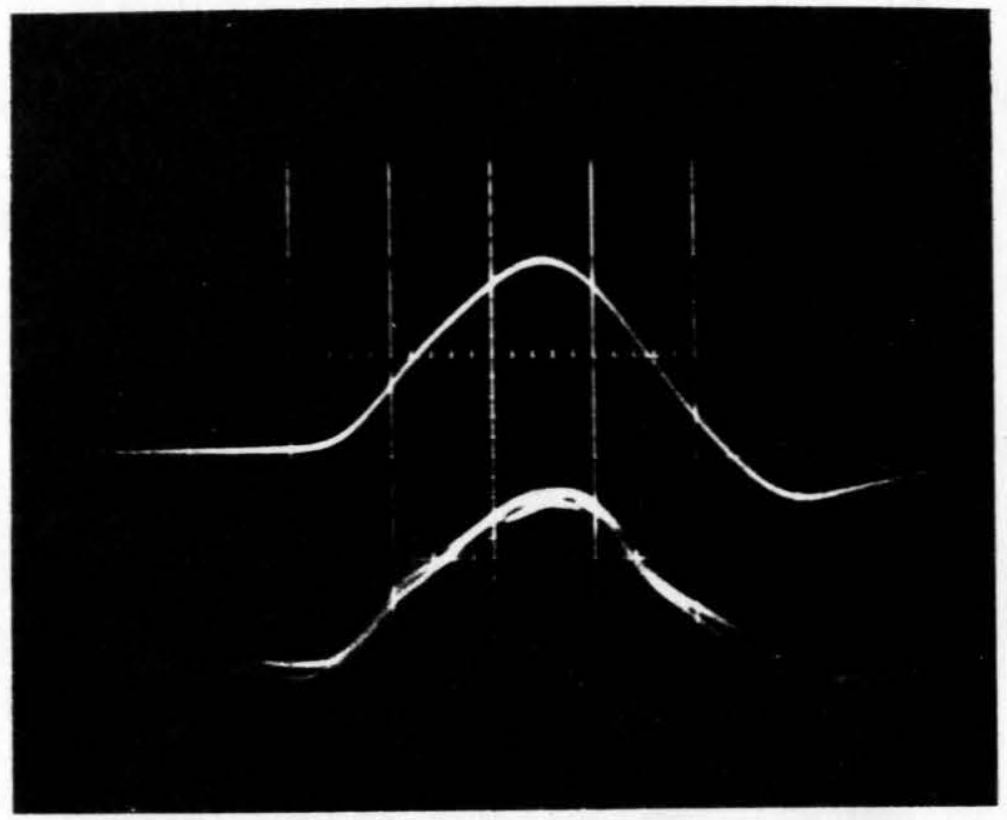

FIGURE 17.

$$
C^{12}(4+)=\alpha_{1} \text { Coincidences }
$$

This is a multiple exposure showing five triggered sweeps of the dual-beam oscilloscope, with the spectrometer current, alpha angle, and delay set to the values appropriate to $C^{12}(4+)-\alpha_{1}$ coincidences. The alpha-particle pulses are on the upper beam, and the recoil-ion pulses on the lower beam. The vertical scale for each beam is $0.5 \mathrm{~V} / \mathrm{cm}$, and the common time base is $20 \mathrm{nsec} / \mathrm{cm}$. Note that the higher gain necessary for the lower energy $\mathrm{C}^{12}$ pulses results in a higher relative noise level. (See text pp. 17, 21.) 


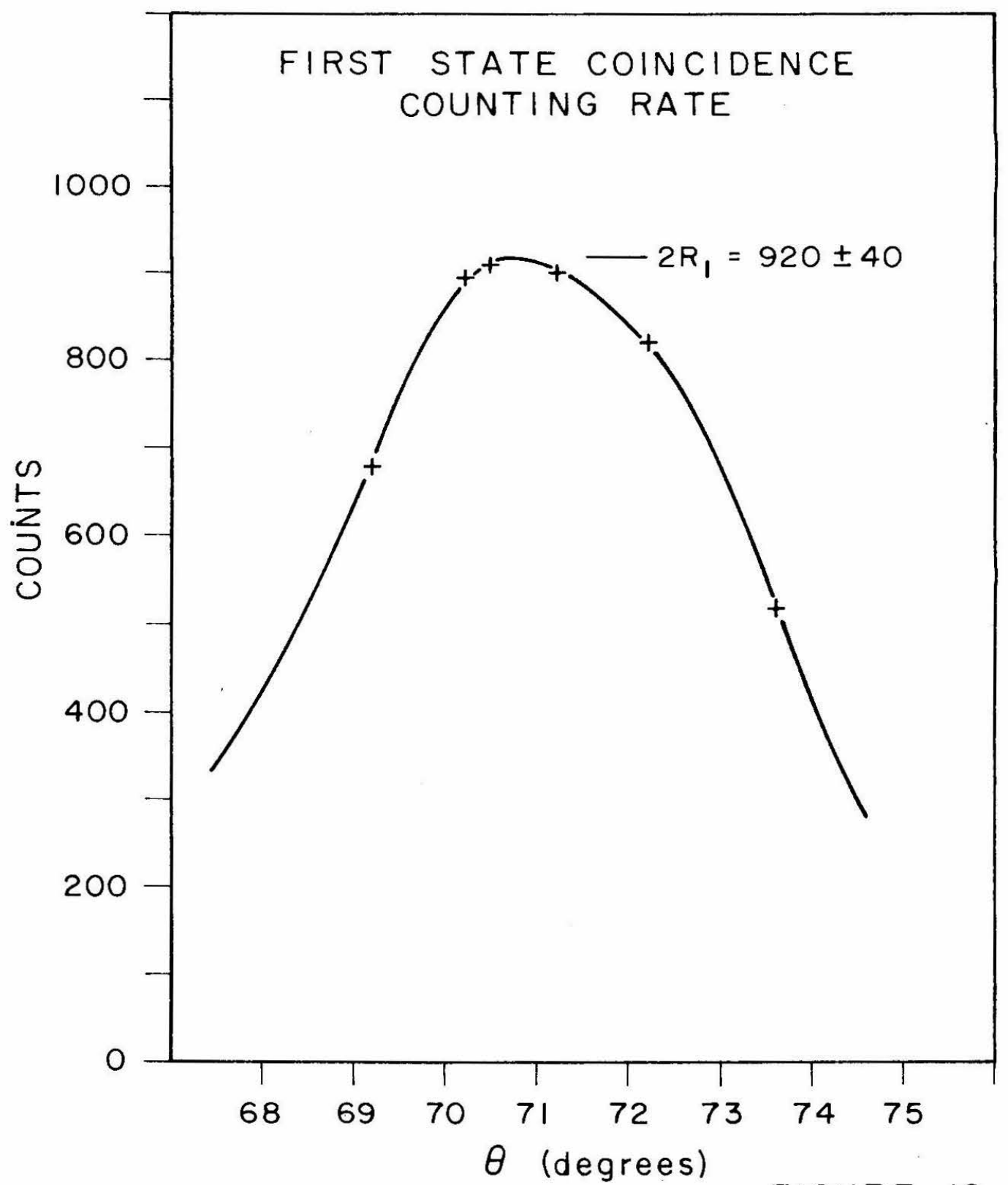

First-State Coincidence Counting Rate

FIGURE 18.

With the spectrometer current and delay set, the alpha counter angle $\theta$ was varied to find the maximum $\mathrm{c}^{12}(4+)-\alpha_{1}$ coincidence counting rate. The curve is normalized to $20,000\left(\alpha_{1}+\alpha_{0}\right)$ alpha particles, and hence the maximum rate is $2 R_{1}$. (See text p. 17.) 


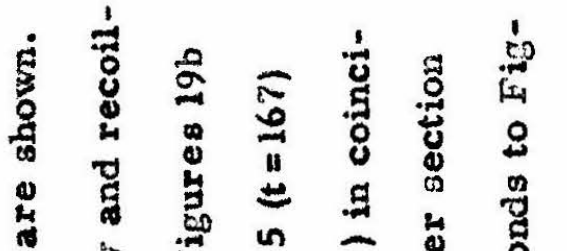

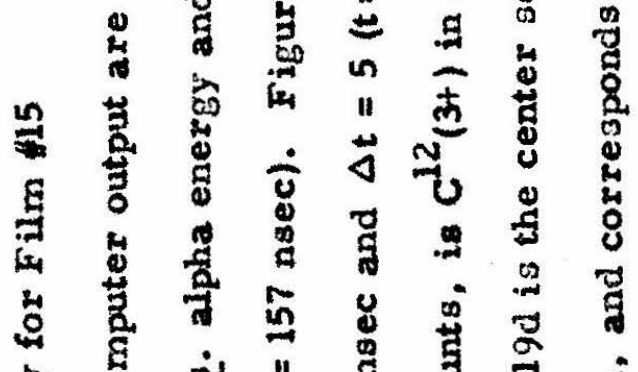

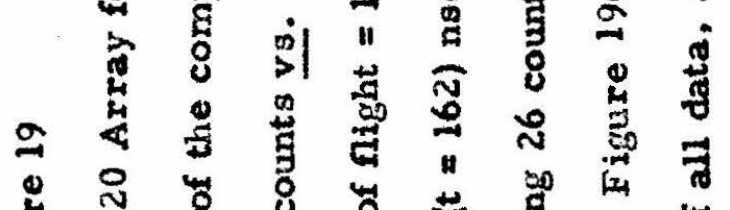

$$
\begin{aligned}
& \text { 总 }
\end{aligned}
$$

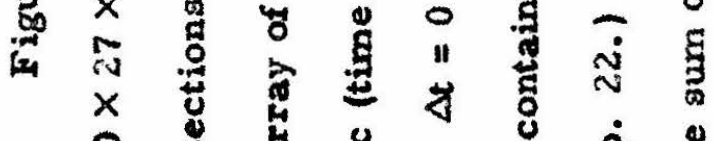

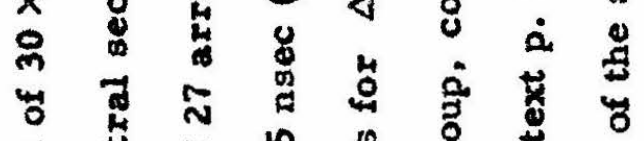

$$
\begin{aligned}
& \text { 泀 } \\
& \text { 葱 }
\end{aligned}
$$

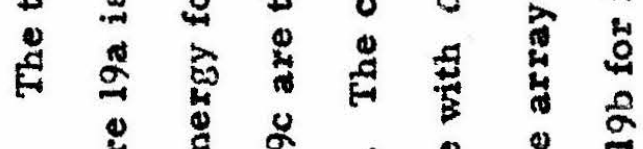

$$
\begin{aligned}
& \text { 离 }
\end{aligned}
$$




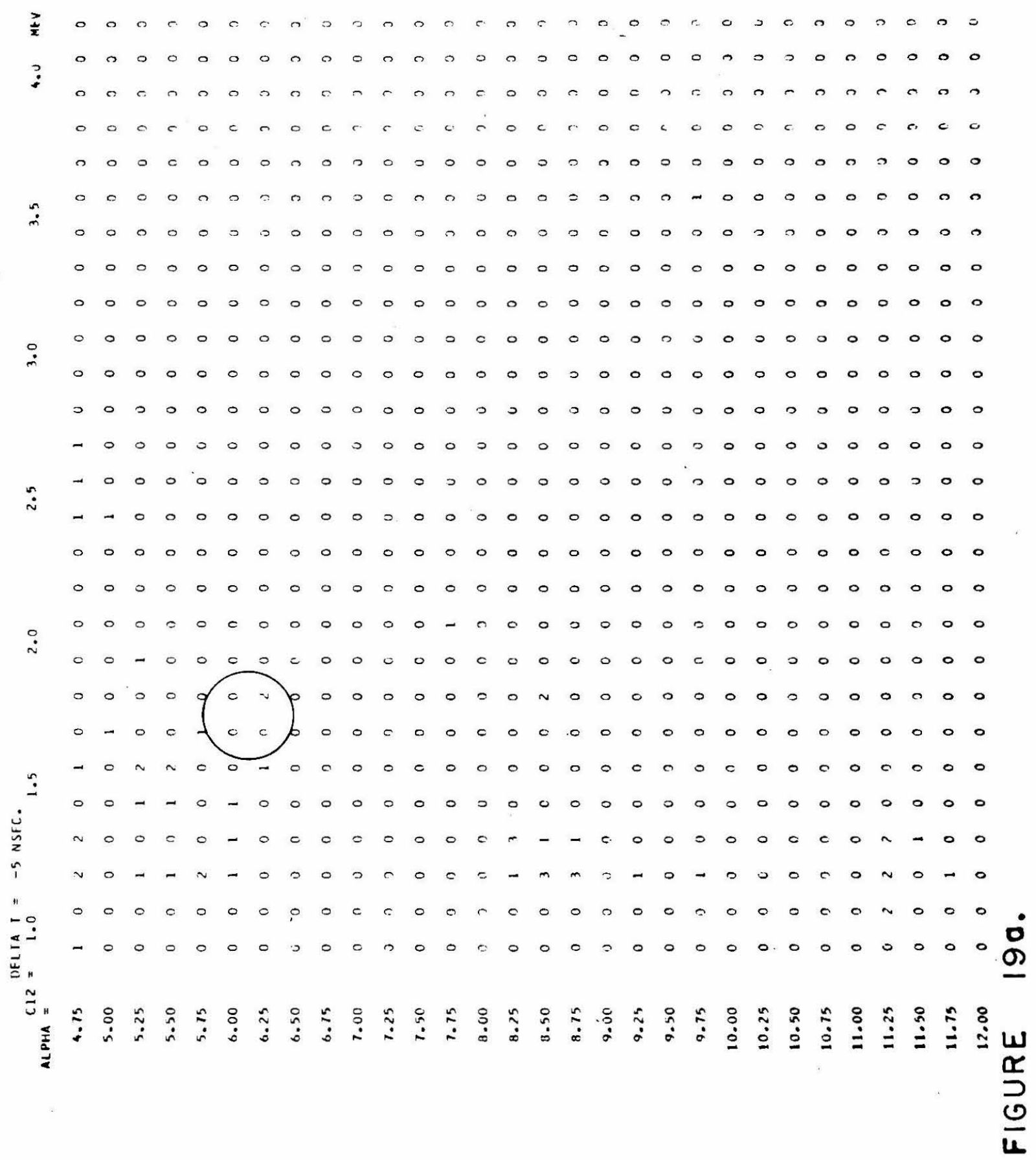




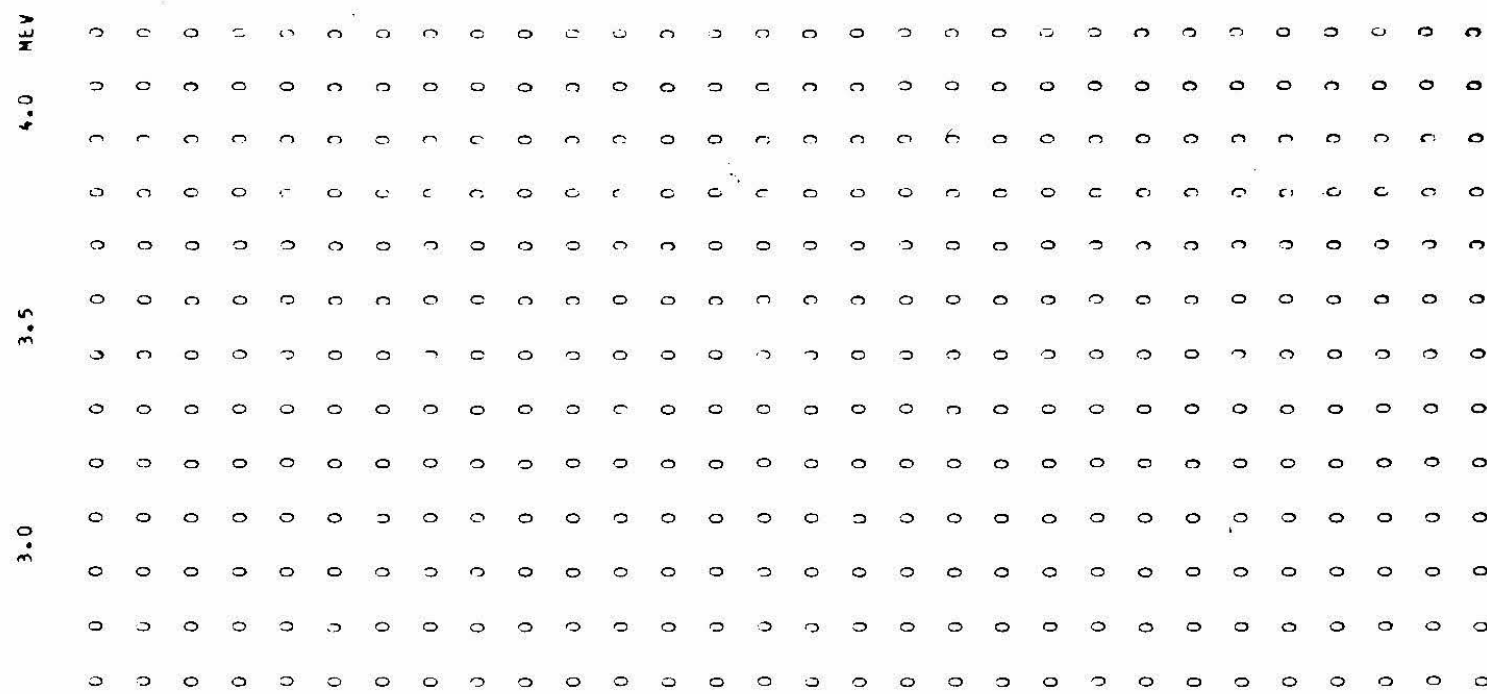

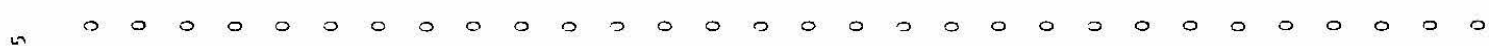

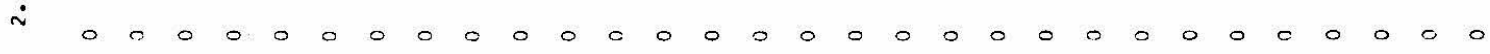

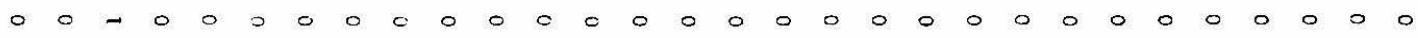

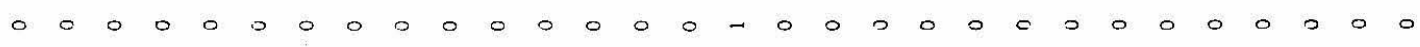
$\stackrel{0}{\sim}$

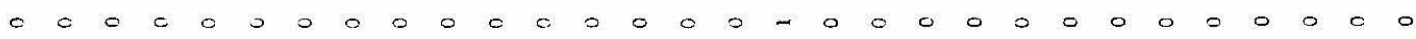

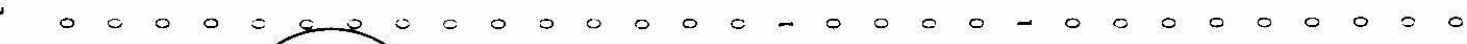

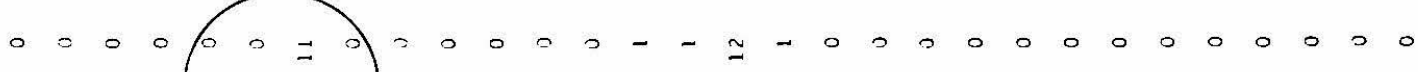

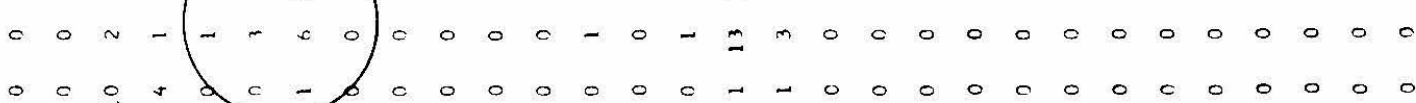

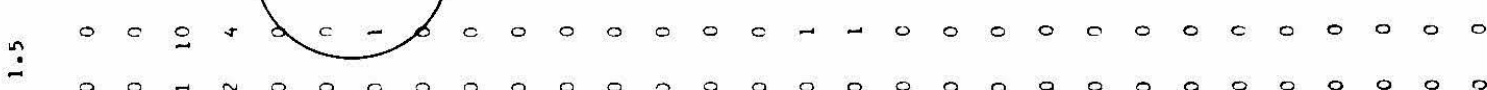
$\dot{j}$
$\tilde{u}$
$\mathbf{z}$
0

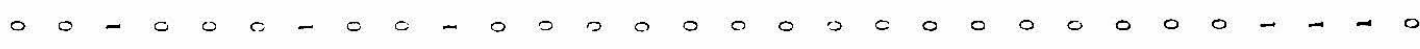

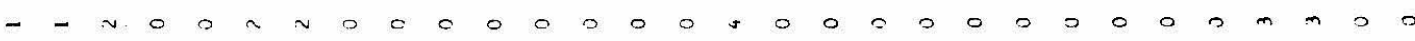

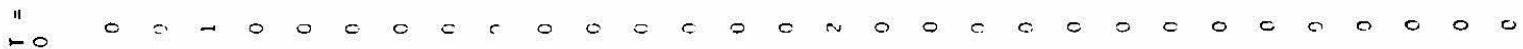

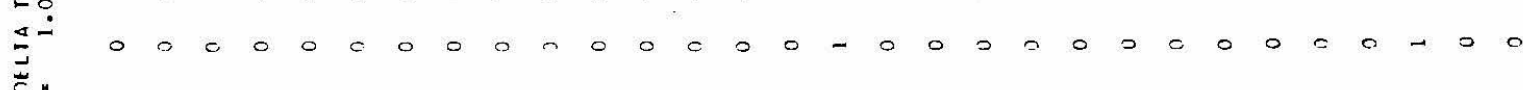

Э. 


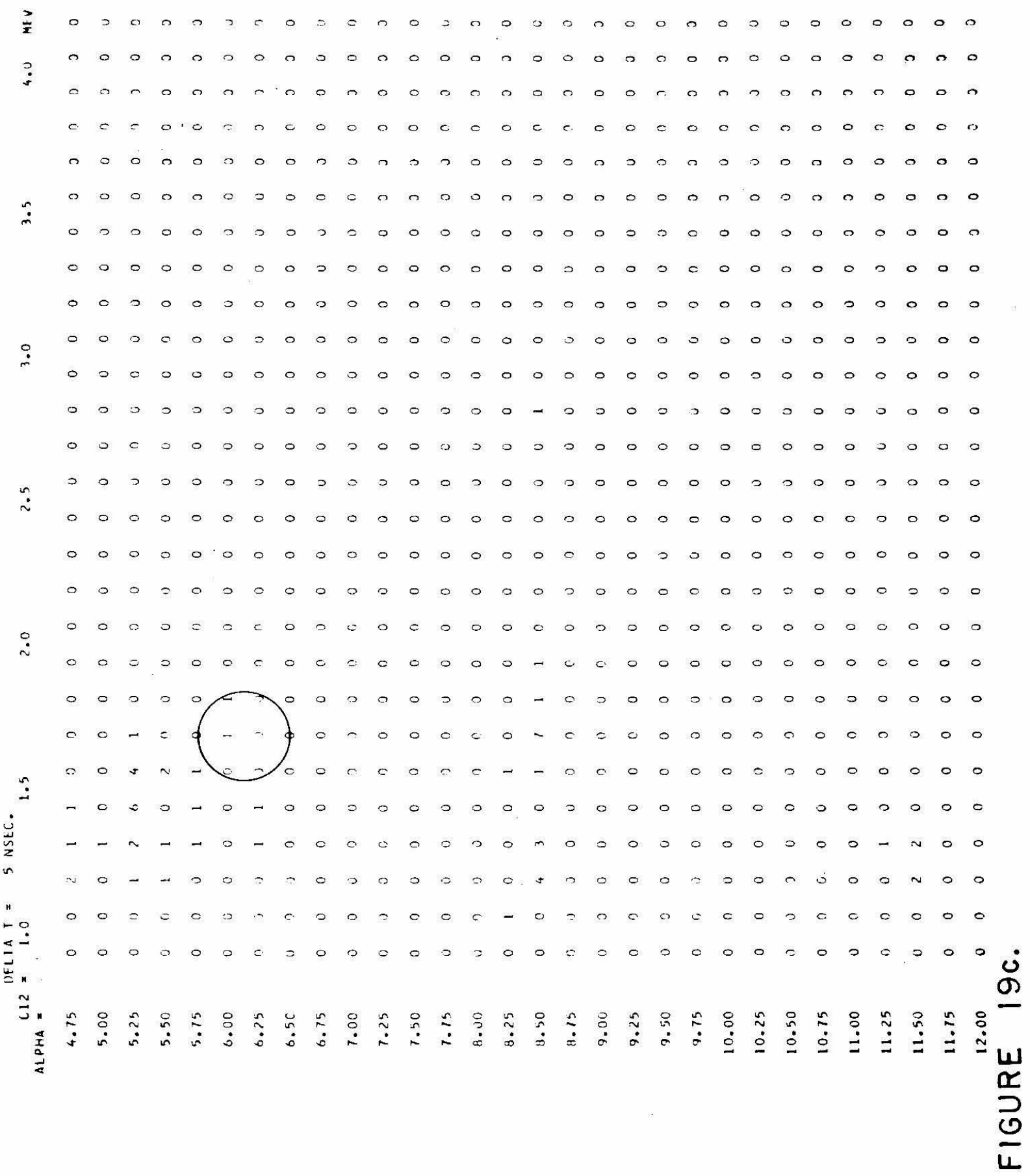




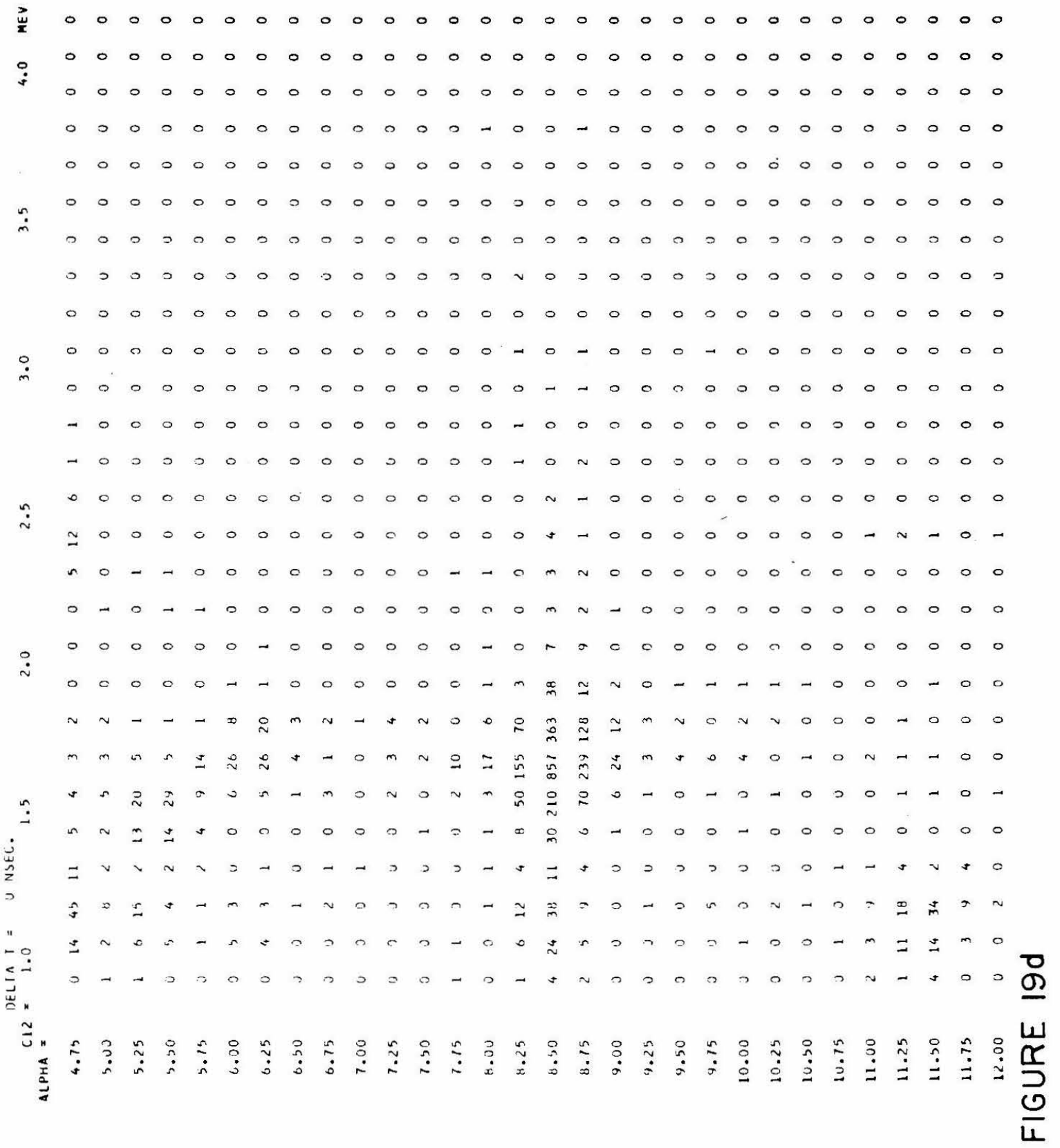




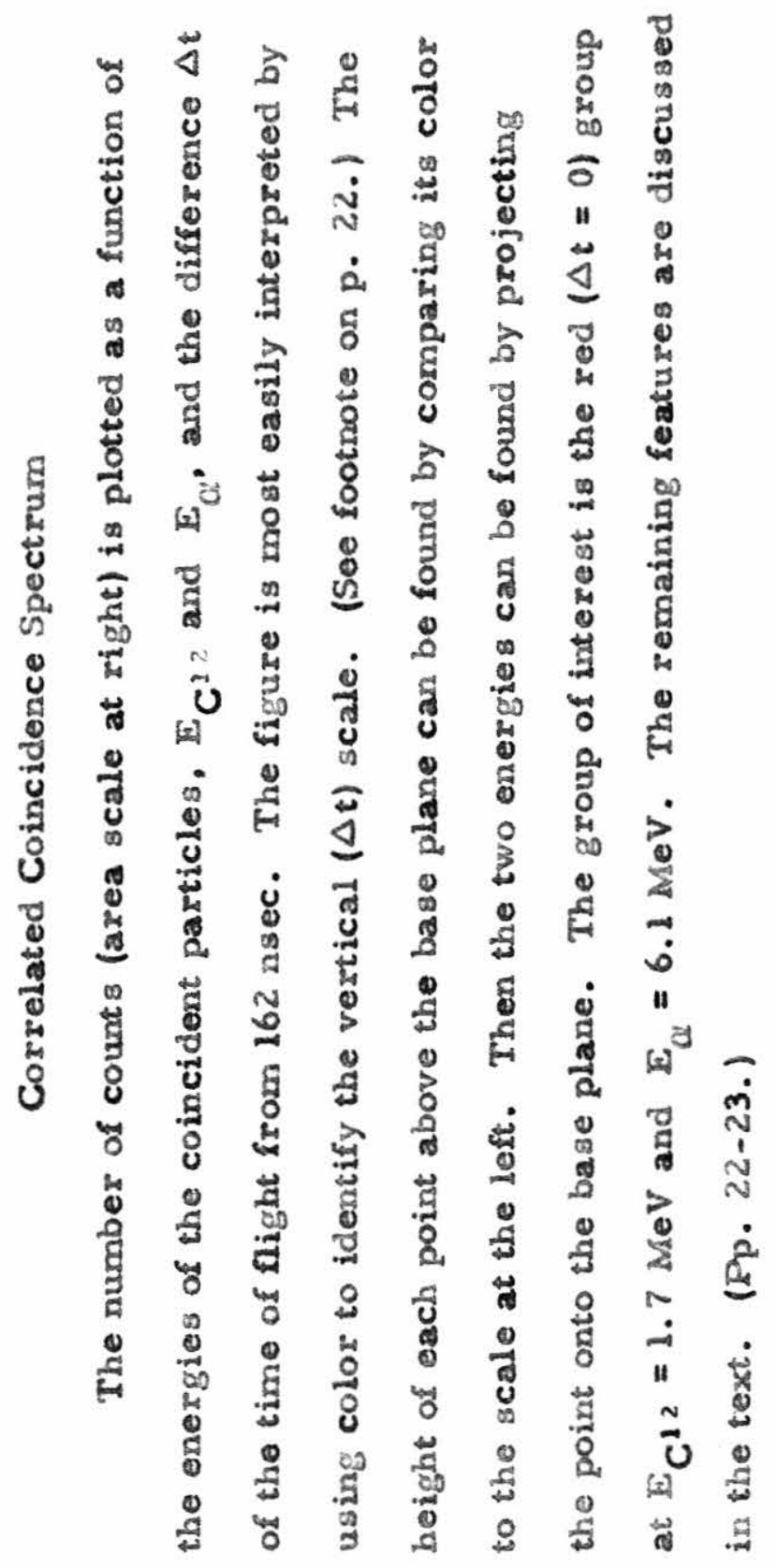




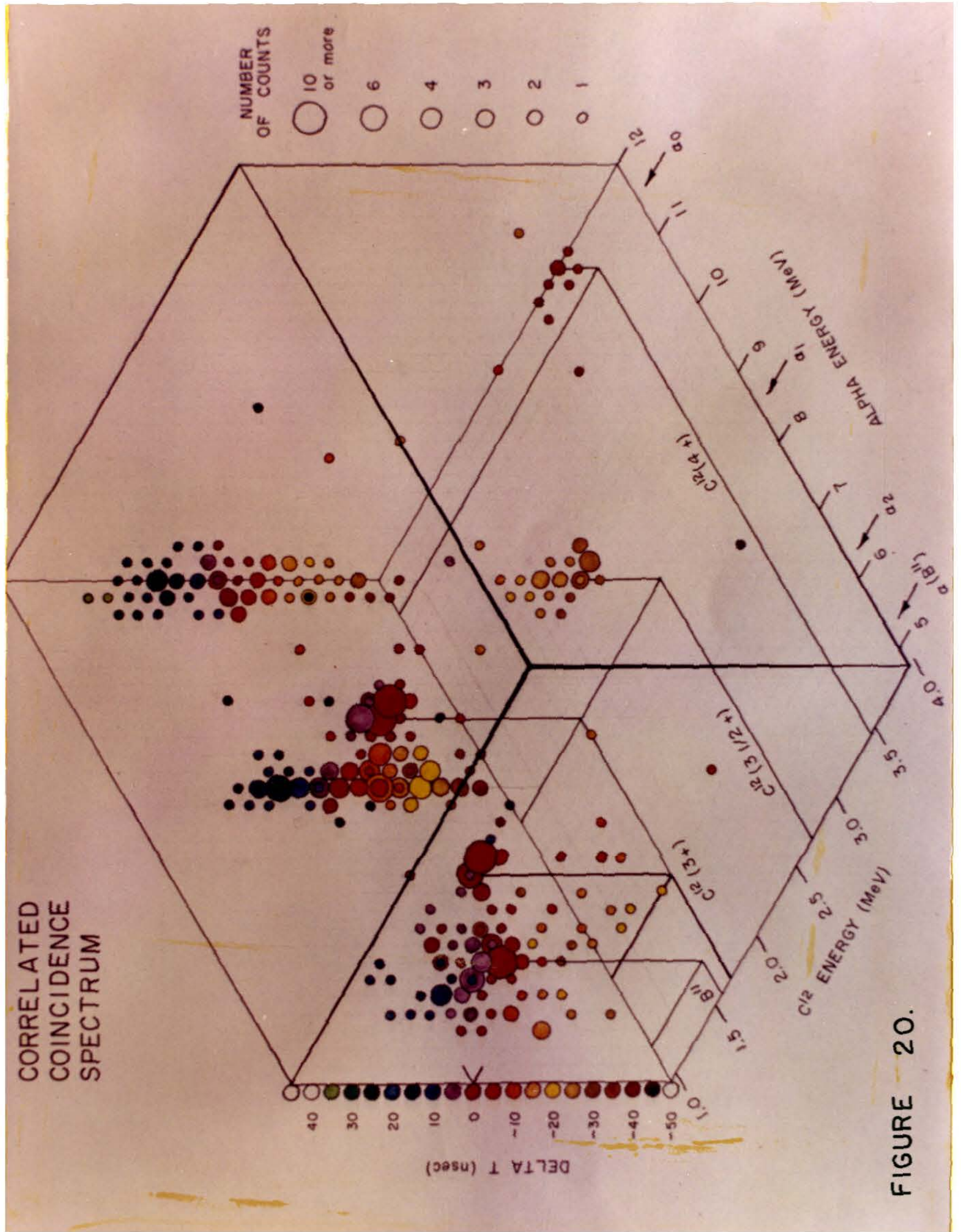




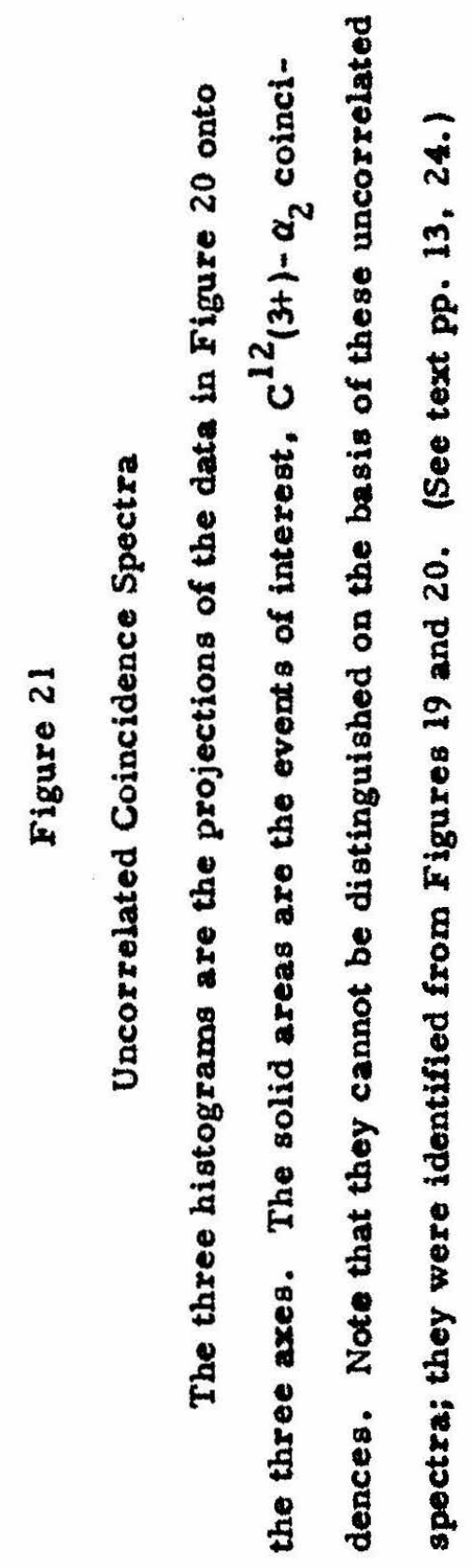


$-93$.

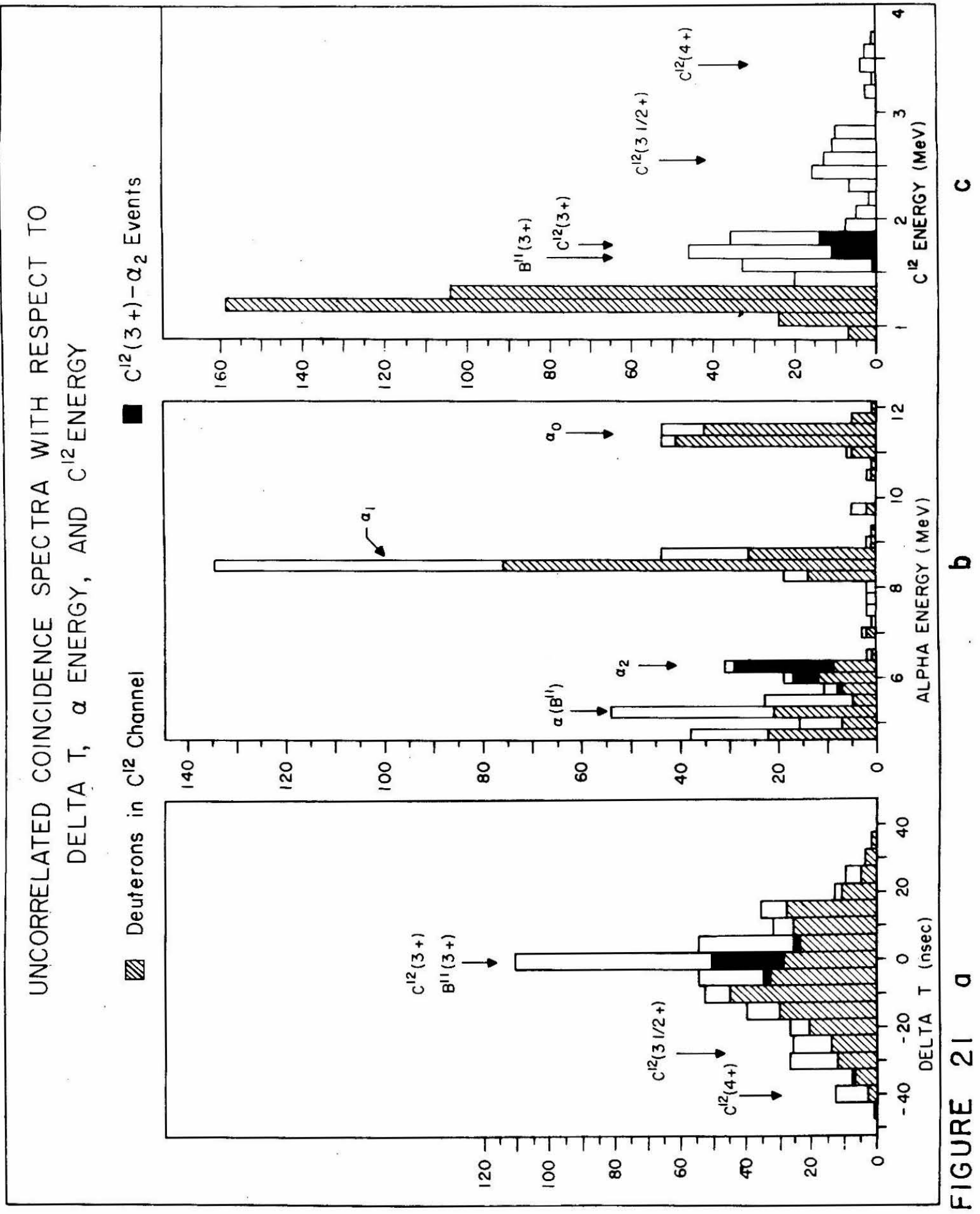




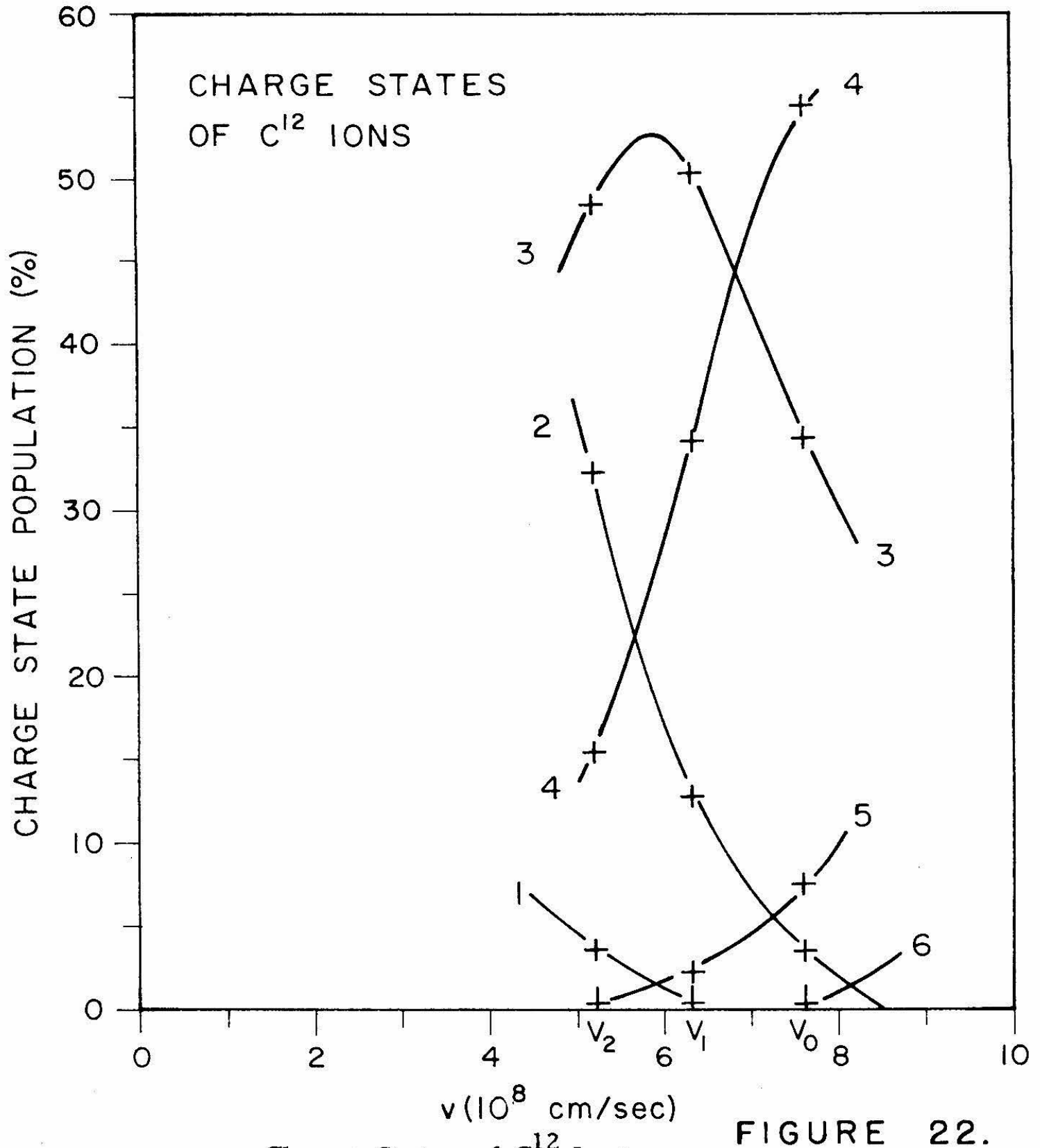

Charge States of $\mathrm{C}^{12}$ Ions

The charge ratios listed in Table VI are plotted vs. the velocity of carbon ions. The three velocities $v_{0}, v_{1}$, and $v_{2}$ correspond to $r e-$ coil- $\mathrm{C}^{12}$ ions from $\mathrm{N}^{14}(\mathrm{~d}, \mathrm{c}) \mathrm{C}^{12}$ reactions involving ground-state, firststate, and second-state events, respectively. (See text p. 30.) 
NOTATION FOR SOLID ANGLE CALCULATIONS

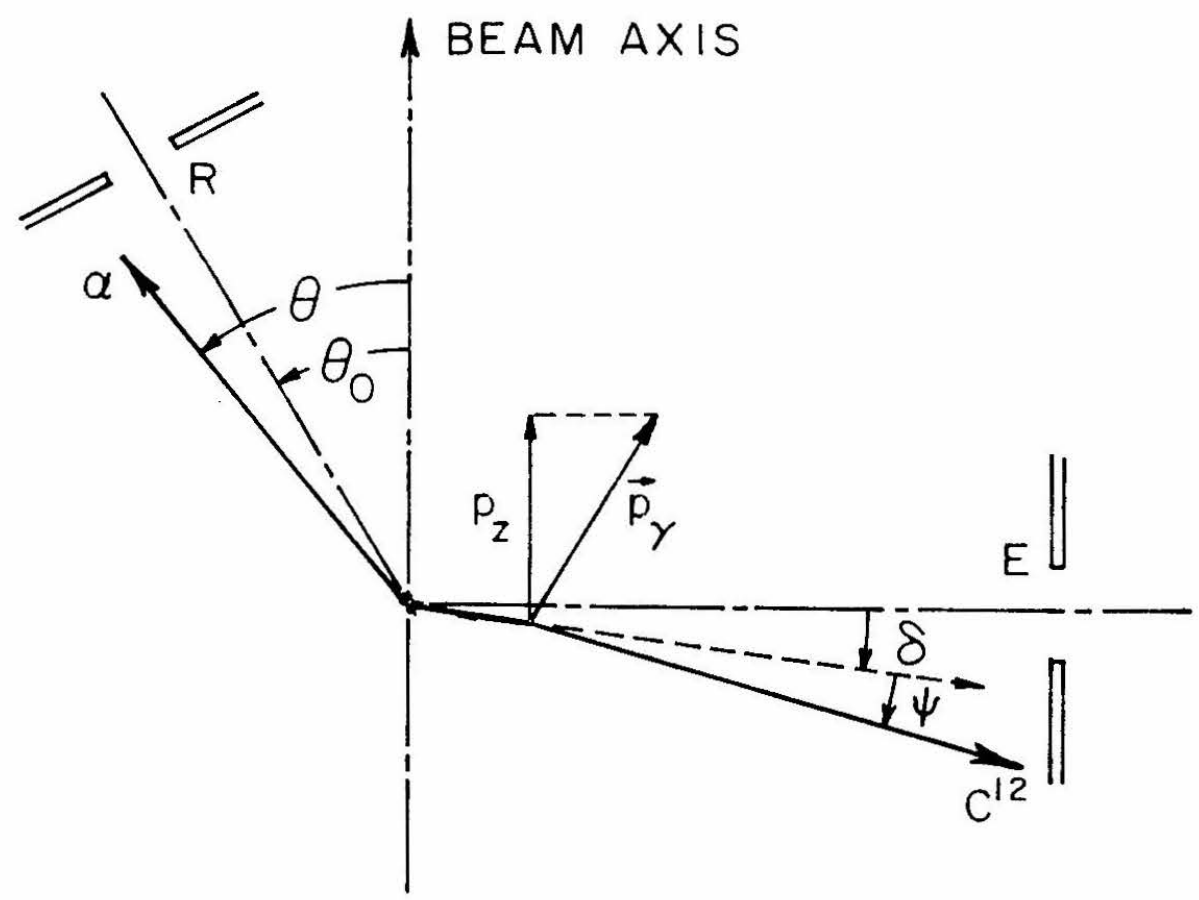

FIGURE 23.

Notation for Solid Angle Calculations

The angle $\theta_{0}$ is the center of the rectangular slit $\mathrm{R}$, and is the alpha angle corresponding to a recoil-ion angle of $90^{\circ}$. Angles $90^{\circ}+\delta$ and $\theta$ are the $C^{12 *}$ and $\alpha$ directions after the $\mathrm{N}^{14}(d, \alpha) \mathrm{C}^{12 *}$ reaction. The recoil ion is deflected by gamma emission; the horizontal projection of the deflection is the angle $\psi$. The component of the gamma momentum vector $\vec{p}_{\gamma}$ parallel to the beam axis is $p_{z}$. (See text $p .31$, Appendix C.) 


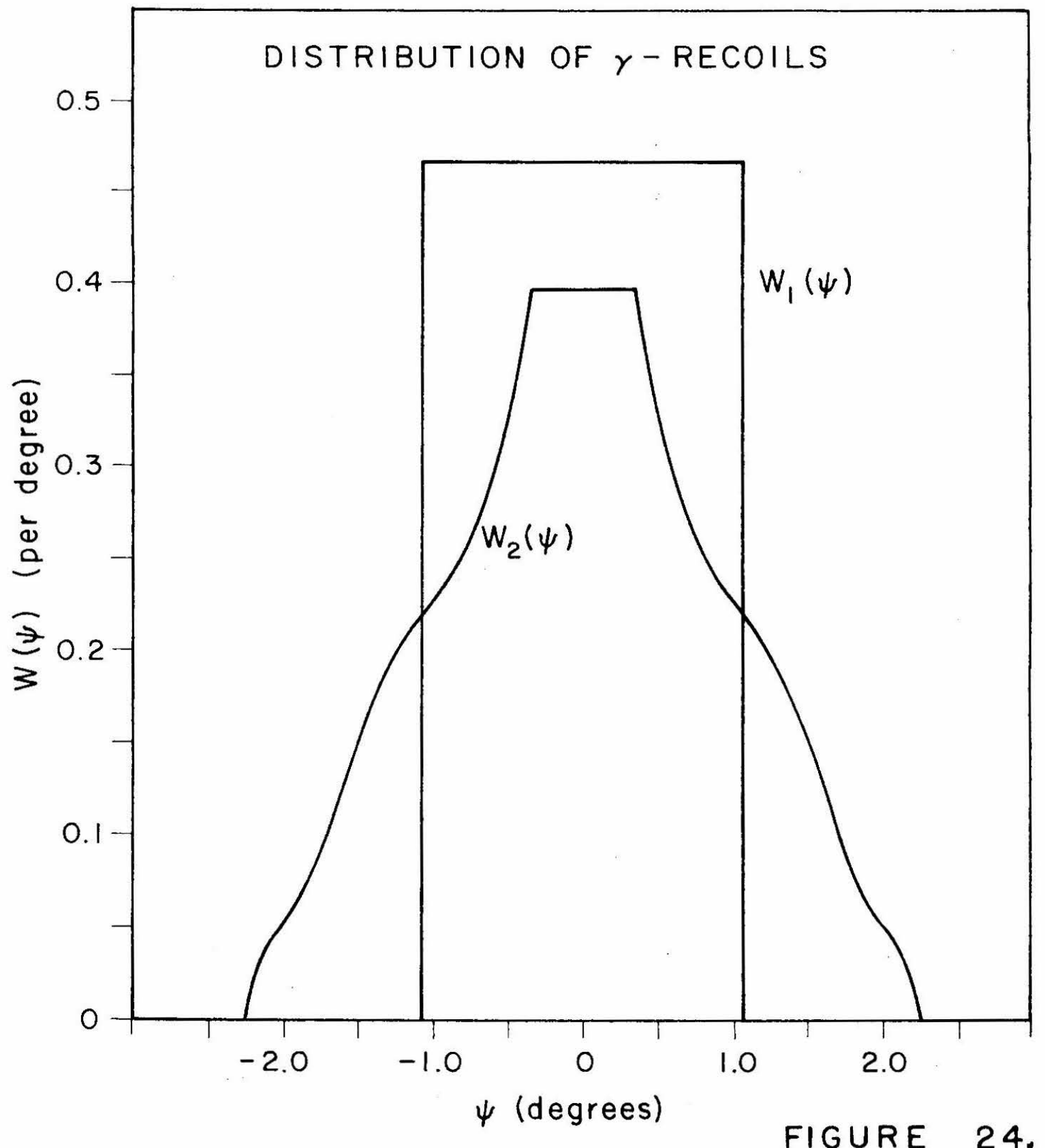

Distribution of $\gamma$-Recoils

W $(\psi) d \psi$ is the probability that the projection in the horizontal plane of the deflection of a recoiling $C^{12 *}$ nucleus by gamma emission will be betweon $\psi$ and $\psi+d \psi$. $W_{1}$ refers to $C^{12}$ nuclei formed in the first excited state, and $W_{2}$ refers to second-state nuclei. These curves also represent the probability distributions for a single component of the gamma momarsum. (See text p. 32, Appendix C.) 


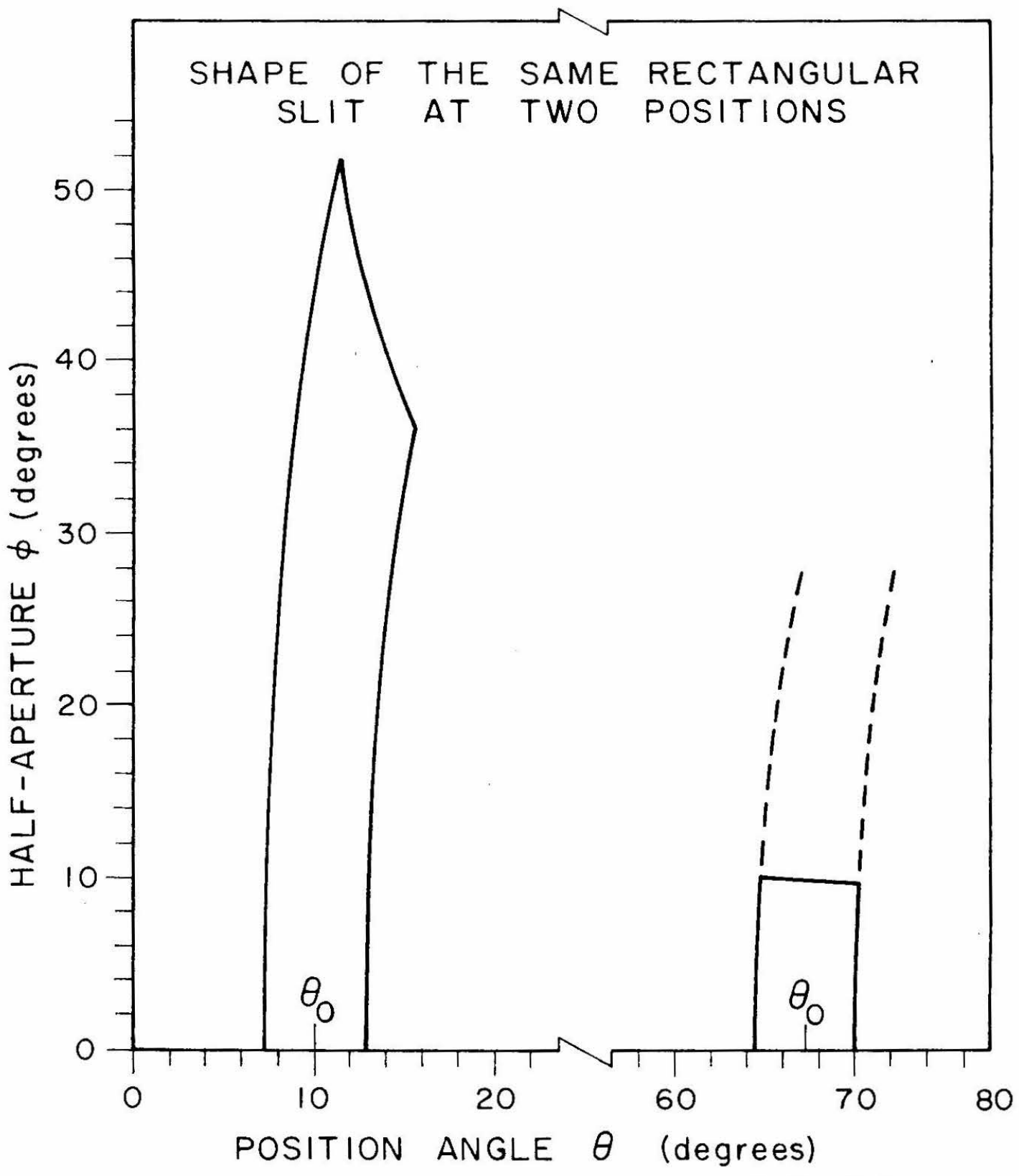

Shape of Rectangular Slit

FIGURE 25.

The shape of the 0.060 -incl wide, 0.200 -inch tall alpha slit is shown as projected on a spkere at kwo different positions. (See text p. 33, Appendix D.) 
$-98-$

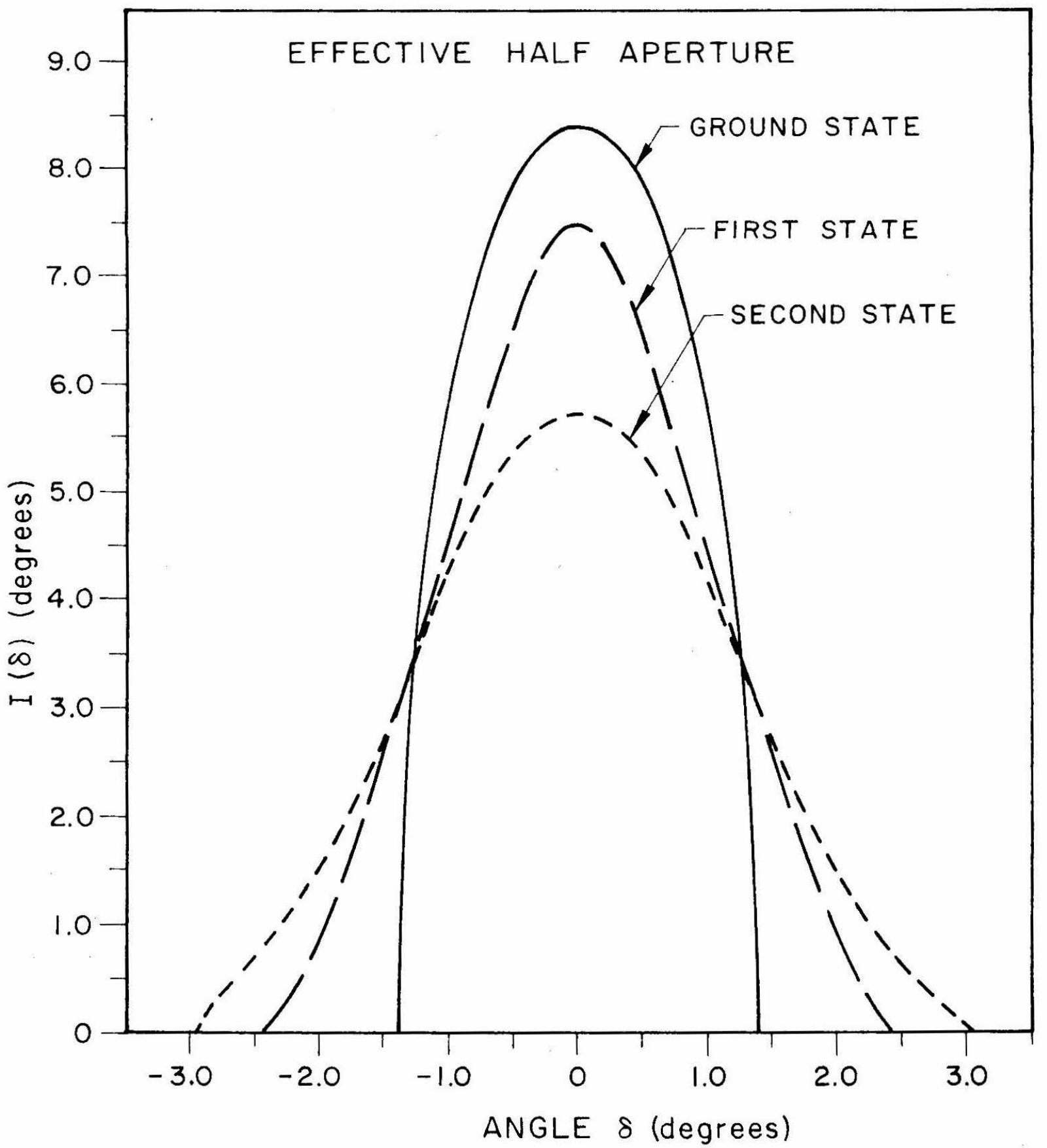

Effective Half Aperiuro

FIGURE 26.

The function $I(\sigma)$ is effectively the probability that a $\mathrm{C}^{12}$ ion initially deviating from $90^{\circ}$ by an angle $\delta$ will be detected in coincidence with its alpha particle. For the ground state this is just the shape of the spectrometer aperture. (See text pp. 33, 35.) 


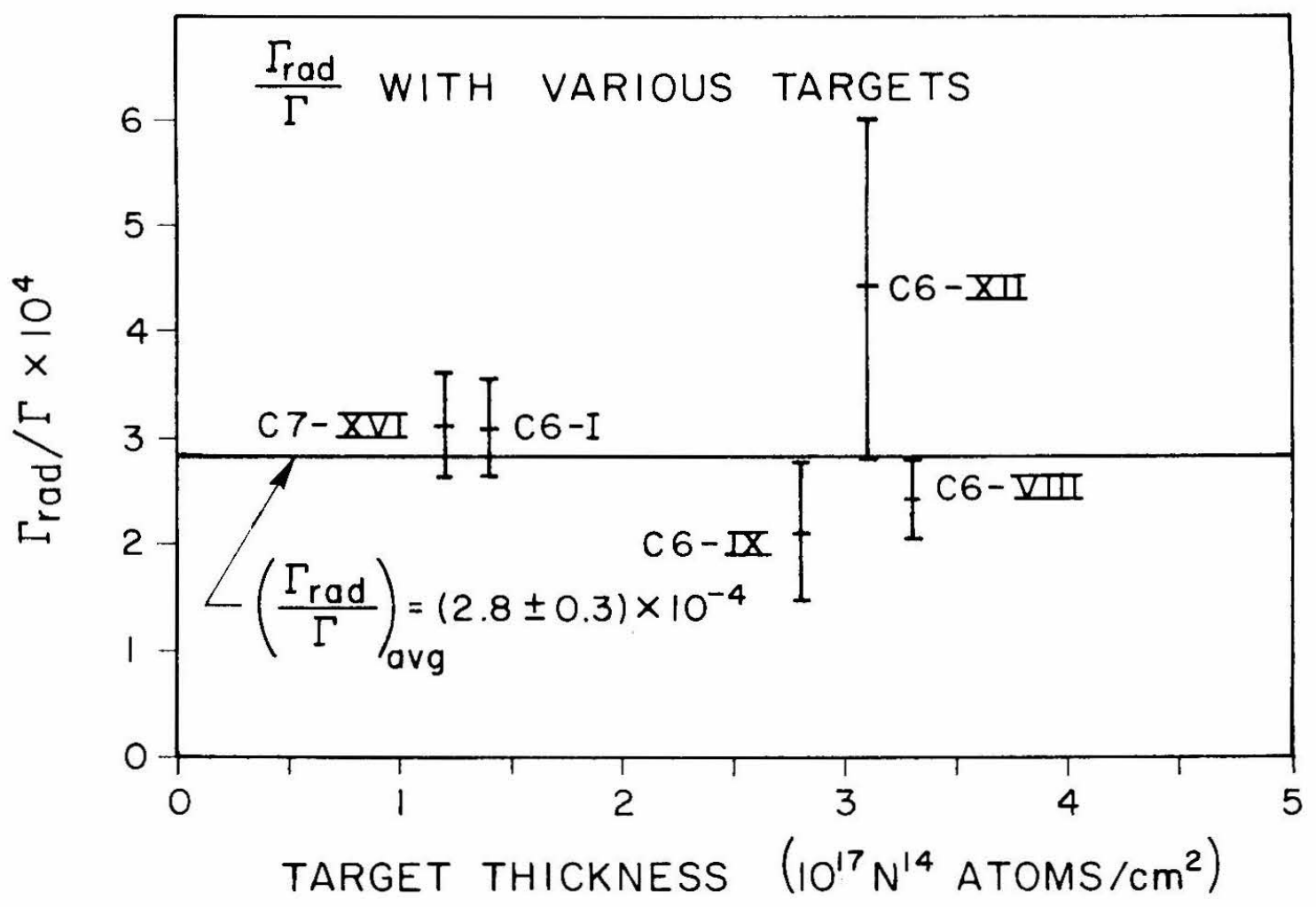

FIGURE 27.

$$
\Gamma_{\text {rad }} / \Gamma \text { with Various Targets }
$$

The sum of the results from each of the five targets used to obtain data are displayed $\mathrm{vs}$. target thickness, to show that target thickness effects have been fairly well accounted for. The average of all data is $\Gamma_{\text {rad }} / \Gamma=(2.8 \pm 0.3) \times 10^{-4}$. (See text p. 36.) 


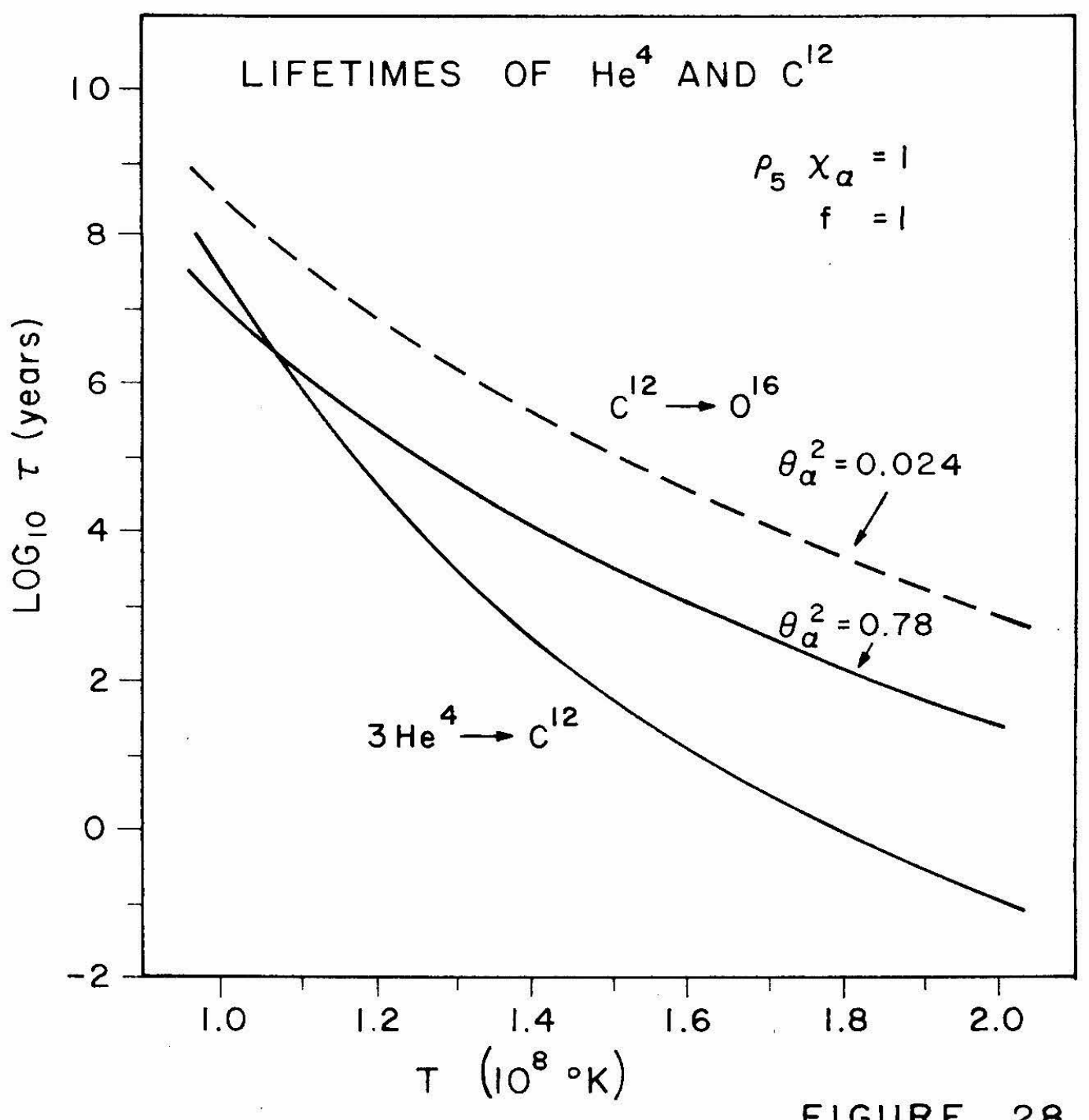

Lifetimes of $\mathrm{He}^{4}$ and $\mathrm{C}^{12}$

The logarithm of the lifetime $7 \alpha^{\left(\mathrm{He}^{4}\right)}$ for a $\mathrm{He}^{4}$ nucleus in the $3 \mathrm{c}$ process is plotted as a function of teriperature for $p_{5}{ }_{c}=1$ and $i=1$. (Curve labeled $3 \mathrm{He}^{4}-\mathrm{C}^{12}$.) Also plotted is the lifetime $T_{U}\left(\mathrm{C}^{12}\right.$ ) of a $\mathrm{C}^{12}$ nucleus undergoing He-burning, for two different assumptions of the width $\theta_{y}^{2}$ of a level in $\mathrm{O}^{16}$. The valie 0.78 is preferred theoretically and astronomically. (See text p. 38.) 